\title{
Synthetic glabridin derivatives mitigate steatohepatitis in a diet- induced biopsy-confirmed non-alcoholic steatosis hepatitis mouse model through paraoxonase-2
}

\author{
Gu-Choul Shin ${ }^{\# 1}$, Hyeong Min Lee ${ }^{\# 2,3,4}$, Na Yeon Kim¹, Sang-Ku Yoo ${ }^{4}$, Yun Sun Park ${ }^{4}$, \\ Hyung Soon Park ${ }^{4}$, Dongryeol Ryu ${ }^{5}$, Kwang Pyo Kim ${ }^{2,3 *}$, and Kyun-Hwan Kim ${ }^{1 *}$
}

${ }^{1}$ Department of Precision Medicine, School of Medicine, Sungkyunkwan University, Suwon 16419, Republic of Korea

${ }^{2}$ Department of Applied Chemistry, Institute of Natural Science, Global Center for Pharmaceutical ingredient Materials, Kyung Hee University, Yongin 446-701, Republic of Korea

${ }^{3}$ Department of Biomedical Science and Technology, Kyung Hee Medical Science Research Institute, Kyung Hee University, Seoul, 02453 Republic of Korea

${ }^{4}$ Glaceum Inc., Suwon, Republic of Korea

${ }^{5}$ Department of Molecular Cell Biology, School of Medicine, Sungkyunkwan University, Suwon 16419, Republic of Korea

\footnotetext{
\# These authors contributed equally to this work.

*To whom correspondence should be addressed:
}

K.P.K (kimkp@khu.ac.kr): Department of Applied Chemistry, Kyung Hee University, Yongin 446-701, Republic of Korea, Tel: +82-31-201-5260

K.H.K (khkim10@skku.edu): Department of Precision Medicine, School of Medicine, Sungkyunkwan University, Suwon 16419, Republic of Korea Tel: +82-31-299-6126 


\begin{abstract}
Limited therapeutic agents have been developed for non-alcoholic steatohepatitis (NASH), a common immunometabolic disease that can progress to hepatic cirrhosis and cancer. Glabridin and its derivatives are potential therapeutics for some metabolic diseases. However, the therapeutic effects of glabridin and its derivatives on NASH and their biological functions are unclear. This study demonstrated the role of synthetic glabridin derivatives (SGDs) in alleviating hepatic steatosis and inflammation in a biopsy-confirmed rodent NASH model. SGDs exerted therapeutic effects by activating autophagy and the antioxidant defense system, which mitigate NASH pathogenesis. The cellular target of HSG4112, an SGD, was paraoxonase 2. These findings will enable the development of novel therapeutics for NASH in the future.
\end{abstract}




\section{Introduction}

Non-alcoholic steatohepatitis (NASH), which is characterized by hepatic inflammation and fibrosis, can progress to hepatic cirrhosis or cancer. ${ }^{1}$ The hepatic accumulation of free fatty acids (FFAs) and toxic lipid intermediates promotes the development of non-alcoholic fatty liver disease (NAFLD) by inducing oxidative stress, mitochondrial dysfunction, and hepatic inflammation. ${ }^{2-4}$ These metabolic disorders along with the consumption of high-calorie diet and sedentary lifestyle are a major public health concern worldwide. ${ }^{5,6}$ Thus, there is an urgent need to develop therapeutic agents for $\mathrm{NASH}$ that can effectively promote toxic lipid metabolite catabolism and mitigate oxidative stress and hepatic inflammation.

Clinical trials have examined various therapeutic drugs for NASH. Semaglutide, a glucagon-like peptide-1 receptor agonist (GLP1-RA), exhibited promising results for the clinical treatment of $\mathrm{NASH}$ in a recent phase 2 trial. However, semaglutide was not effective against the fibrosis stage. ${ }^{7}$ The mechanism underlying the pharmacological activity of semaglutide involves the suppression of gastric emptying and food intake through GLP-1R-dependent regulation of the physiology in both the hypothalamus and hindbrain. Semaglutide treatment decreases bodyweight and liver weight, alleviates hepatic steatosis, and inhibits the onset of NAFLD in mouse models. ${ }^{8}$ However, hepatocytes do not express the canonical GLP-1R, ${ }^{9}$ and the expression of GLP-1R has not been conclusively established in other liver cells. The role of GLP-1RA in the attenuation of $\mathrm{NASH}$ is unclear. The elucidation of a novel mechanism distinct from that of GLP-1RA may provide novel insights into the alleviation of NASH.

Glabridin has important applications in the food, dietary supplement, and cosmetic industries. However, the application of glabridin is limited owing to its low water 
solubility, low bioavailability, and unpredictable stability. ${ }^{10}$ Orally administered glabridin exhibits various bioactivities, including antioxidant, anti-inflammatory, and estrogen-like activities, and promotes lipid catabolism. ${ }^{11,12}$ Thus, glabridin and its derivatives are potential therapeutic agents for immunometabolic diseases, such as NASH although the underlying mechanisms have not been completely elucidated.

The chemical stability and oral bioavailability of synthetic glabridin derivatives (SGDs), which were recently synthesized by our group, are higher than those of glabridin. In this study, the therapeutic effect of SGDs on NASH was evaluated using a rodent model. Additionally, the underlying mechanism of action (MOA) of SGDs was elucidated. 


\section{Results}

\section{HSG4112 alleviates steatohepatitis in the biopsy-confirmed NASH mouse model}

The therapeutic efficacy of SGDs in progressive steatohepatitis was examined using an amylin diet-induced obesity (AMLN-DIO) mouse model, which exhibits severe inflammation and liver fibrosis. The liver of mice fed on AMLN diet for 37 weeks was biopsied 4 weeks before SGD treatment to confirm the development of NASH. Mice were administered with SGDs during the last 6 weeks of AMLN diet supplementation (Supplementary Fig. 1a). Hematoxylin and eosin (H\&E) staining and oil red $\mathrm{O}$ staining revealed that steatosis in the HSG4112-treated and HSG4113-treated groups was significantly alleviated when compared with that in the vehicle-treated group (Fig. 1a). HSG4112 and HSG4113 mitigated the AMLN diet-induced liver fibrosis (Sirius red staining and Lgals3 and Acta2 immunostaining) and intrahepatic monocyte infiltration (Adgre1 immunostaining). Consistently, biochemical analysis revealed that HSG4112 and HSG4113 mitigated the AMLN diet-induced upregulation of serum and hepatic cholesterol levels and plasma levels of Fgf21 and Lep (steatosis markers), Tnfa, and II10 (inflammatory markers), and alanine aminotransferase (ALT), aspartate aminotransferase (AST), and alkaline phosphatase (ALP) (hepatic injury markers) but did not affect the II5, II6, and Cxcl1 levels (Fig. 1b and Supplementary Fig. 2a).

The NASH-related phenotypes before SGD treatment were compared with those at week 6 post-SGD treatment. Compared with that at the baseline, the NAFLD activity score (NAS), which indicates the degree of lobular inflammation, steatosis, and ballooning degeneration, was lower at week 6 post-HSG4112 treatment. In contrast, the NAS of the HSG4113-treated group was not significantly different at baseline and 
week 6 post-treatment (Fig. 1c-e, Supplementary Fig. 3). Compared with that in the vehicle-treated group, the number of mice exhibiting decreased NAS was significantly higher in the HSG4112-treated group and similar in the HSG4113-treated group. HSG4112 and HSG4113 did not alleviate hepatic ballooning and fibrosis (Fig. 1f and Supplementary Fig. 2b). Additionally, HSG4112 significantly decreased the liver weight and bodyweight (Fig. 1g-h). The HSG4112-treated, HSG4113-treated, and vehicle groups exhibited similar daily food intake (Fig. 1i). These findings indicate that HSG4112 alleviated NASH.

HSG4112 alleviates hepatic inflammation and NASH without decreasing the appetite

A phase 2 clinical trial revealed that semaglutide alleviates $\mathrm{NASH}{ }^{13}$ Thus, the therapeutic effects of HSG4112 and semaglutide on NASH were comparatively analyzed (Supplementary Fig. 1b). Similar to the 6-week treatment regimen, longterm (10 weeks) treatment with HSG4112 alleviated hepatic steatosis in the AMLN-DIO mouse model (Fig. 2a) and downregulated the cholesterol levels (Fig. 2b) to levels observed in the semaglutide-treated group. Semaglutide and HSG4112 mitigated liver fibrosis and intrahepatic monocyte infiltration (Fig. 2a) and downregulated Ccl2 (an inflammatory marker) and ALT, AST, and ALP (hepatic injury markers) (Fig. 2b).

The mice were stratified into HSG4112-treated, semaglutide-treated, and vehicle groups based on the NASH phenotypes at the baseline. The NASH phenotypes at the baseline were compared with those at week 10 post-drug treatment. Treatment with HSG4112 and semaglutide for 10 weeks significantly decreased the NAS (Fig. 2c). Additionally, the number of mice with decreased NAS in the HSG4112-treated and 
semaglutide-treated groups was significantly higher than that in the vehicle-treated group. In contrast to semaglutide, HSG4112 mitigated lobular inflammation (Fig. 2d). However, HSG4112 and semaglutide did not mitigate hepatic fibrosis (Supplementary Fig. 4). HSG4112 and semaglutide significantly decreased the liver weight and bodyweight after treatment for 10 weeks (Fig. 2e-f and Supplementary Fig. 5a). The initial bodyweight reduction rate in the semaglutide-treated group was higher than that in the HSG4112-treated group. However, the final bodyweight was similar in both groups (Fig. 2f). Additionally, HSG4112 and semaglutide alleviated the AMLN dietinduced changes in body composition and adiposity (Fig. $\mathbf{2 g - h}$ and Supplementary Fig. 5b-c). In contrast to HSG4112, semaglutide markedly decreased the daily food intake (Fig. 2i and Supplementary Fig. 5d). These findings indicate that HSG4112 alleviates NASH without decreasing the appetite and mitigates hepatic inflammation.

\section{Semaglutide and HSG4112 differentially affected the hepatic lipid profiles}

Next, the mechanisms underlying the therapeutic effects of HSG4112 and semaglutide were examined. The hepatic lipid profiles of the HSG4112-treated and semaglutidetreated groups were examined using liquid chromatography-electrospray ionizationtandem mass spectrometry (LC-ESI-MS/MS). Principal component analysis (PCA) revealed that the lipid profiles of the HSG4112-treated, semaglutide-treated, and vehicle-treated groups exhibited distinct clustering. This indicated that the MOA of HSG4112 was distinct from that of semaglutide (Fig. 3a). Volcano plot analysis revealed that HSG4112 downregulated 70 lipid species, including cholesterol esters (CEs, 24:0 and 18:4) and triacylglycerols (TAGs, 42:2), while semaglutide downregulated 128 lipid species, mainly TAGs (42:2 and 44:4) (Fig. 3b). HSG4112 
significantly upregulated 37 lipid species, including sphingomyelins (SMs) and phosphatidylcholines (PCs), while semaglutide upregulated 31 lipid species, including PCs and FFAs. The relative contents and saturation levels of various glycerolipids, including monoacylglycerol (MAG), diacylglycerol (DAG), and TAG, were markedly downregulated in the HSG4112-treated and semaglutide-treated groups (Fig. 3c and Supplementary Fig. 6). Additionally, HSG4112 downregulated FFAs, ceramide (Cer), and lysophospholipid (LPL) species, including lyso-phosphatidylcholine (LPC), lysophosphatidylethanolamine (LPE), lyso-phosphatidylinositol (LPI), and lysophosphatidylglycerol (LPG), whereas semaglutide downregulated phosphatidic acid.

The levels of 78 individual lipid species were significantly different between the HSG4112-treated and semaglutide-treated groups (Fig. 3d and Supplementary Fig. 7). HSG4112 downregulated most individual MAGs, whereas semaglutide downregulated most individual DAGs and TAGs. In contrast to semaglutide, HSG4112 downregulated most individual LPCs, LPEs, and LPGs and upregulated individual SMs. HSG4112 distinctively downregulated some FFAs, including long-chain FFAs, whereas semaglutide upregulated most FFAs, including saturated and unsaturated FFAs. The levels of cholesterol species and PCs were not markedly different between the HSG4112-treated and semaglutide-treated groups but were significantly different from those in the vehicle-treated group. Lipidome analysis revealed that the MOA of HSG4112 was distinct from that of semaglutide.

Transcriptomic analysis was performed to examine the expression levels of lipid catabolic enzymes. The mRNA levels of genes encoding enzymes involved in FFA uptake, CE biosynthesis from FFAs, cytidine diphosphate (CDP)-DAG biosynthesis from DAG, lysophosphatidic acid (LPA) biosynthesis from DAG, DAG biosynthesis from 
MAG and TAG, and ceramide-1-phosphate (CerP) biosynthesis from Cer were not significantly different between the HSG4112-treated and semaglutide-treated groups (Fig. 3e). However, the levels of Agpat5 and Agpat1, which encode enzymes involved in phosphatidic acid biosynthesis from LPA, were significantly downregulated in the semaglutide-treated group but not in the HSG4112-treated group. The levels of $L p /$ and Pla2g15, which are involved in LPC biosynthesis from PC, were significantly downregulated in the semaglutide-treated group. Furthermore, the expression of Pitpnb, which is involved in the release of extracellular PC, was significantly upregulated in the semaglutide-treated group but not in the HSG4112-treated group.

Two clusters of lipid species were strongly correlated with alleviating NASH in the HSG4112-treated group. The levels of these lipid species in the HSG4112-treated and semaglutide-treated groups were comparatively analyzed. A cluster of 10 lipid species, including PE, LPC, LPE, FFA, and Cer, was positively correlated with NASH alleviation in the HSG4112-treated group. In the semaglutide-treated group, 8 lipid species containing phosphatidic acid and SM were positively correlated with NASH alleviation (Fig. 3f). The LPC, LPE, FFA, and Cer levels were positively correlated with the alleviation of hepatic inflammation in the HSG4112-treated group but not in the semaglutide-treated group. These results suggest that the regulatory effects of HSG4112 on lipid catabolism are distinct from those of semaglutide.

\section{HSG4112 modulates the autophagic and anti-inflammatory pathways}

To investigate the mechanisms underlying HSG4112-mediated NASH alleviation, the gene expression levels were analyzed using RNA sequencing (RNAseq). Of the 22028 genes, the levels of approximately $10 \%$ of genes in the semaglutide-treated (2244 
genes) and HSG4112-treated (2413 genes) groups were different from those in the vehicle group (Fig. 4a). To identify the specific target genes of the drugs, the differentially expressed genes (DEGs) between the HSG4112-treated and semaglutidetreated groups were analyzed. Among the meta-signature genes, HSG4112 and semaglutide co-regulated 1146 genes, which represent common gene signatures of the drugs for NASH alleviation (Fig. 4b). Furthermore, HSG4112 and semaglutide specifically altered the expression of 1267 and 1098 genes, respectively, which represent the drug-specific gene signatures. Gene Ontology (GO) analysis revealed that 384 upregulated overlapping genes were significantly enriched in biological oxidation, metabolism of lipids, steroid biosynthesis, and fatty acid degradation, whereas 762 downregulated overlapping genes were significantly enriched in extracellular matrix organization, immune system, liver fibrosis, and inflammation signaling (Fig. 4c-d and Supplementary Fig. 8a-d). Meanwhile, 656 genes specifically upregulated in the HSG4112-treated group were related to glutathione metabolism, branched-chain amino acid degradation, and autophagy, while 491 genes specifically upregulated in the semaglutide-treated group were related to bile acid and arachidonic acid metabolism (Fig. 4c and e). Furthermore, 611 genes specifically downregulated in the HSG4112-treated group were related to cell adhesion molecules, antigen processing and presentation, and MAPK signaling pathway, whereas 607 genes specifically downregulated in the semaglutide-treated group were related to Rho GTPase cycle and fibrin clot formation (Fig. 4c and f).

Next, the levels of phosphoproteins and their corresponding total proteins in the HSG4112-treated and semaglutide-treated groups were comparatively analyzed. Common signatures of upregulated phosphoproteins and their corresponding total 
proteins were associated with oxidative phosphorylation, steroid hormone biosynthesis, and fatty acid degradation (Fig. $\mathbf{4 g}$ ). Meanwhile, common signatures of downregulated phosphoproteins and their corresponding total proteins were associated with the phagosome, focal adhesion, and tight junction. Compared with that in the semaglutidetreated group, the phosphorylation of autophagy-linked AMPK signaling pathwayrelated components was upregulated, while that of B cell receptor, chemokine, and $\mathrm{T}$ cell receptor signaling pathway-related components was downregulated in the HSG4112-treated group.

To examine the network of biological processes related to specific gene and protein signatures in the HSG4112-treated group, functional interaction networks were constructed (Fig. 4h). The autophagy-linked biological processes were the major targets of HSG4112. Insulin receptor signaling, oxidative stress response, mTOR signaling, and AMPK signaling are the upstream signals of autophagy. HSG4112 activated autophagy initiation signaling (Ulk1 and Ulk2), autophagosome biogenesis (Rb1cc1, Wipi1, Wipi2, and Atgs), and autophagy maturation (Map1Lc3b, Stx17, Tecpr1, and Lamp2). These findings suggest that HSG4112 regulates lipid catabolism through the induction of the autophagy pathways.

\section{HSG4112 activates autophagy/mitophagy and attenuates mitochondrial oxidative stress under lipotoxic conditions}

Next, the effect of HSG4112 on alleviating lipotoxicity in hepatocytes was examined. HSG4112 significantly mitigated FFA-induced lipid accumulation in the hepatocytes (Fig. 5a). Omics data indicated that HSG4112 promoted autophagy, which promotes the degradation of lipid droplets and the release of FFAs. Lipid droplets and FFAs are 
then oxidized through mitochondrial fatty acid oxidation (FAO), which results in the alleviation of lipotoxicity. ${ }^{14}$ Hence, the autophagic flux was examined by monitoring the conversion of LC3B-I to LC3B-II and the degradation of Sqstm1. Consistent with the results of the mechanistic study on hepatic lipid profiles (Fig. 3c and e), compared with those in the FFA-treated cells, the LC3B levels were upregulated and the Sqstm1 levels were downregulated in the HSG4112-treated cells (Fig. 5b). HSG4112-induced autophagy activation was confirmed using the autophagy indicator tandem fluorescence-tagged LC3B. Compared with that in the control and FFA-treated groups, the number of cells with autophagic flux (red fluorescent protein (RFP)) was higher in the HSG4112-treated group (Fig. 5c). HSG4112-induced autophagic flux was confirmed using the lysosomal fusion inhibitor bafilomycin A (BFA). The number of autophagosomes in the BFA-treated cells was markedly higher than that in the control or FFA-treated cells as evidenced by the accumulation of both green fluorescent protein (GFP) and RFP puncta (Fig. 5d). Map1lc3b and Becn1 immunostaining analyses revealed that HSG4112 significantly promoted autophagy activation in the mouse liver (Fig. 5e).

The analysis of genes involved in electron transport chain revealed that HSG4112 upregulated mitochondrial FAO (Supplementary Fig. 8f). FAO is reported to enhance reactive oxygen species (ROS) generation. ${ }^{15}$ Hence, the effect of HSG4112 on mitochondrial oxidative stress generated during FAO was examined. HSG4112 markedly downregulated palmitic acid (PA)-induced mitochondrial superoxide to the level observed in the control group (Fig. 5f). Additionally, HSG4112 significantly downregulated lipid peroxidation in the NASH mouse liver and PA-treated hepatocytes (Fig. 5g). Mitophagy is a quality control mechanism that protects the cells against 
cytotoxic ROS production by clearing damaged mitochondria. ${ }^{16}$ HSG4112 suppressed the PA-induced mitophagy inhibition by upregulating Prkn/Pink1, Bnip3, and Bnip3I. Additionally, HSG4112 promoted the mitophagic flux by upregulating LC3B-II and downregulating Sqstm1 (Fig. 5h). The mitophagy rates in hepatocytes stably expressing mt-Kemia, a mitochondrial dual-excitation ratiometric fluorescent protein reporter of mitophagy, were analyzed. ${ }^{59}$ Consistent with results of immunoblotting analysis, mitophagy activation in the HSG4112-treated group was higher than that in the PA-treated or control groups as evidenced by enhanced mitochondrial localization to acidic lysosome (Fig. 5i). ${ }^{17}$ These findings indicate that HSG4112 activates the autophagy-FAO axis and attenuates oxidative stress by activating mitophagy.

\section{HSG4112 activates autophagy and alleviates oxidative stress through Pon2}

To elucidate the mechanism of HSG4112, the intracellular localization of HSG4112 was examined using Alexa 488 azide-conjugated propargylated HSG4112, which was synthesized using the Click-iT reaction. Fluorescence analysis revealed that HSG4112 primarily localized to the mitochondria (Fig. 6a). Hepatic gene function analysis indicated that HSG4112 targets mitochondrial functions (Supplementary Fig. 8e). To identify the target protein of HSG4112, biotin-labeled HSG4112 was synthesized using the Click-iT reaction. Cell lysates were subjected to the pull-down assay and chemical proteome analysis. The enriched GO terms for 130 HSG4112-bound proteins in the mitochondria were related to the regulation of overall mitochondrial energy metabolism, including oxidative phosphorylation, FAO, tricarboxylic acid cycle, and oxidative stress homeostasis (Supplementary Fig. 9a-b). HSG4112 interacted with paraoxonase 2 (Pon2), which is reported to hydrolyze lipid peroxide and alleviate oxidative stress (Fig. 
6b)..$^{18}$ To confirm the specific interaction, a competitive pull-down assay was performed by pre-incubating the cell lysates with free HSG4112. Free HSG4112 dosedependently decreased the interaction between HSG4112 and Pon2. This was consistent with the results of studies using purified recombinant Pon2 (rPon2) protein (Fig. 6c). The interaction between HSG4112 and Pon2 may modulate the Pon2 esterase and lactonase activities. Hence, the Pon2 activity in HSG4112-treated cells was examined. HSG4112 mitigated the PA-induced downregulation of Pon2 activity although it did not affect the Pon2 expression (Fig. 6d). Next, the ability of HSG4112 to specifically activate Pon2 was examined as Pon1 and Pon3 also exhibit esterase and lactonase activities in hepatocytes. HSG4112 did not affect the esterase and lactonase activities in Pon2 knockdown (KD) cells, which suggested that HSG4112 specifically activates Pon2 (Fig. 6e). Additionally, HSG4112 mitigated the oxidized linoleic acidinduced downregulation of rPon2 activity in vitro (Fig. 6f). These results indicate that HSG4112 suppresses lipid peroxide-induced inhibition of Pon2 activity. Furthermore, Pon2 is reported to exhibit antioxidant activities through the reduction of superoxide generated by CoQ10 in the mitochondria, which leads to the downregulation of lipid peroxidation. ${ }^{19-21}$ Thus, the effect of HSG4112 on mitochondrial superoxide was examined. HSG4112 markedly downregulated mitochondrial superoxide production in wild-type cells but not in Pon2 KD cells (Fig. 6g). Consistently, HSG4112 did not downregulate lipid peroxidation in Pon2 KD cells (Fig. 6h), which indicated that Pon2 activity is crucial for the antioxidant effect of HSG4112. The intrinsic function of Pon2 in autophagy/mitophagy activation is unclear. However, the potential role of Pon2 in HSG4112-mediated activation of autophagy/mitophagy was examined in this study. Pon2 KD inhibited autophagy/mitophagy under basal conditions (Fig. 6i-j) as 
evidenced by Sqstm1 upregulation and Prkn and Bnip3I downregulation. The effect of HSG4112 on FFA-induced or PA-induced autophagy/mitophagy was abolished in Pon2 KD cells. These results indicate that Pon2 contributes to the HSG4112-mediated activation of autophagy. HSG4112 did not decrease lipid accumulation in Pon2 KD cells (Fig. 6k). These findings suggest that HSG4112 activates autophagy/mitophagy and alleviates lipotoxicity in hepatocytes through Pon2. 


\section{Discussion}

Glabridin is reported to be a potential therapeutic for metabolic diseases, such as obesity and diabetes mellitus. ${ }^{22,23}$ However, the effect of glabridin and SGDs on NASH severity has not been examined. In this study, a clinically relevant biopsy-confirmed $\mathrm{NASH}$ mouse model was used to accurately examine the therapeutic effect of SGDs on $\mathrm{NASH}^{24,25}$ The anti-inflammatory effects of HSG4112 were higher than those of semaglutide. Moreover, HSG4112 mitigated the AMLN diet-induced adverse effects on the overall physical attributes, including bodyweight loss, liver weight loss, and enhanced lean mass, without decreasing the appetite. HSG4112 markedly reprogrammed the hepatic transcription and metabolism associated with lipid catabolism, hormone biosynthesis, protein anabolism, redox homeostasis, and immune response. GLP-1RAs, such as semaglutide may indirectly alleviate $\mathrm{NASH}$ as the canonical GLP-1R is not expressed in hepatocytes. ${ }^{9}$ In contrast to semaglutide, HSG4112 can be orally administered. Therefore, HSG4112 is a promising therapeutic for $\mathrm{NASH}$ and directly protects hepatocytes.

Lipidomic studies have demonstrated that $\mathrm{NASH}$ is associated with a marked alteration in the hepatic lipidome, which is correlated with the disease progression. ${ }^{26,27}$ The changes in the levels of glycerolipids, phospholipids, and fatty acids are closely associated with the progression of $\mathrm{NASH}$. In particular, $\mathrm{NASH}$ is correlated with the downregulation of plasma PS, PE, PI, PC, and SM contents ${ }^{28,29}$ and upregulation of LPLs. The accumulation of LPC, an important mediator of hepatic lipotoxicity, disrupts mitochondrial integrity and promotes the release of pro-inflammatory molecules. ${ }^{30-32}$ Patients with NASH are characterized by increased levels of saturated FAs and 
decreased levels of polyunsaturated FAs. ${ }^{33,34}$ Unsaturated FAs mitigate the saturated FFA-induced hepatic lipotoxicity. ${ }^{35-37}$ Thus, lipidome profile analysis can aid in evaluating the therapeutic efficacy of drugs against NASH. In this study, the levels of individual lipid species in the lipidome were markedly different between the HSG4112treated and semaglutide-treated groups. HSG4112 upregulated SM and PC species, whereas semaglutide upregulated only some PC species but did not affect the levels of SM species. In this study, the dissipation of LPC, LPE, and LPG in the HSG4112treated group was markedly higher than that in the semaglutide-treated group. Additionally, HSG4112 downregulated most FFA species, especially glycerolipids. Thus, these effects of HSG4112 may be closely associated with the alleviation of hepatic steatosis and inflammation. While semaglutide promoted the accumulation of some saturated FAs in the liver, it also increased the levels of various unsaturated FAs. Therefore, semaglutide may also effectively alleviate FFA-induced hepatic lipotoxicity. However, further studies are needed to examine the potential side effects of semaglutide-induced upregulation of LPLs. These findings indicate that HSG4112 protects against hepatic lipotoxicity by altering the levels of particular lipid species in the hepatic lipidome.

Autophagy is important for the maintenance of liver homeostasis. ${ }^{38}$ Metabolic disorders are characterized by markedly decreased autophagy activity, which leads to enhanced lipid accumulation, mitochondrial dysfunction, oxidative stress, and inflammation. ${ }^{39-41}$ In particular, autophagy promotes lipid catabolism by breaking down lipid droplets and protecting the hepatocytes from inflammation and oxidative stress. ${ }^{42-}$ 44 Transcriptome and proteome data demonstrated that HSG4112 activated the 
autophagic pathways (autophagy initiation, autophagy/mitophagy assembly, and autophagy maturation). HSG4112 markedly downregulated lipid accumulation through autophagy activation in hepatocytes. In NAFLD, the overloading of saturated FFAs into mitochondria promotes mitochondrial dysfunction through the accumulation of ROS in hepatocytes, which exacerbates lipotoxicity and inflammation in the liver. The levels of free radicals generated from FAO are higher than those generated from glucose catabolism under lipotoxic conditions. ${ }^{15,45}$ Consistent with these previous findings, PA treatment increased FAO and enhanced the production of mitochondrial superoxide in hepatocytes in this study. However, HSG4112 decreased oxidative stress to the level observed in the control, upregulated FAO, and decreased lipid peroxidation. These results suggest that HSG4112 alleviates oxidative stress and promotes lipid catabolism by inducing autophagy. Mitophagy clears damaged mitochondria resulting from oxidative stress under pathological conditions. ${ }^{46-49}$ Additionally, mitophagy maintains mitochondrial homeostasis under physiological conditions. ${ }^{50}$ HSG4112 induced mitophagy activation and alleviated oxidative stress, which indicated that it can induce mitophagy independent of oxidative stress. The phospho-proteome data demonstrated that HSG4112 induces autophagy/mitophagy activation through AMPK signaling, which is the major signaling pathway for mitophagy activation in metabolic diseases. ${ }^{45,51}$ Thus, HSG4112 alleviates NASH severity, hepatic steatosis, oxidative stress, and inflammation by activating autophagy.

The identification of the drug target is essential to understand the MOA and determine the clinical dose regimen based on the drug-target engagement. Chemicalprotein interactome data demonstrated that Pon2 was a specific target protein of 
HSG4112. Pon2 is not reported to be associated with NASH in clinical studies. However, Pon2 has a critical role in the pathogenesis of NASH in animal models. ${ }^{52,53}$ In the liver of the NASH rat model, Pon2 expression is upregulated and PON2 activity is downregulated through oxidative inactivation. ${ }^{52}$ In the NASH mouse model, PON2 deficiency decreases energy expenditure and oxidative capacity, which are etiological factors for obesity, an important risk factor for $\mathrm{NASH}^{53}$ Thus, PON2 is a potential therapeutic target for $\mathrm{NASH}$.

The PON family comprises the following three members: PON1, PON2, and PON3. ${ }^{54}$ PON2, which exhibits esterase and lactonase activities, is expressed in most tissues, including the liver. ${ }^{55,56}$ Additionally, PON2 is primarily localized to the mitochondria and endoplasmic reticulum, which are major sites of oxidative stress, and exert antioxidant and anti-inflammatory effects. ${ }^{57,58}$ Therefore, PON2 deficiency promotes mitochondrial dysfunction by increasing oxidative stress. ${ }^{19}$ Mitochondrial localization of HSG4112 indicates that it may be closely associated with the mitochondrial Pon2. HSG4112 promotes Pon2 activity without affecting its expression. Additionally, HSG4112 upregulates FAO and decreases the production of mitochondrial superoxide through Pon2. Furthermore, the antioxidant effect of HSG4112, which downregulated total lipid peroxidation, is dependent on Pon2 activity. Therefore, HSG4112 may exert therapeutic effects by promoting Pon2 activity. Pon2 KD was hypothesized to induce autophagy/mitophagy activation as it increased oxidative stress in hepatocytes. However, Pon2 KD inhibited autophagy/mitophagy under basal and lipotoxic conditions. HSG4112-induced autophagy/mitophagy activation was diminished in Pon2 KD cells, which led to lipid accumulation. Thus, 
HSG4112 alleviates NASH through Pon2 and by activating autophagy/mitophagy, promoting lipid metabolism, and maintaining redox homeostasis.

In summary, these findings indicate that HSG4112-mediated alleviation of hepatic injury was correlated with increased FAO and autophagy and decreased lipid accumulation, inflammation, and mitochondrial oxidative stress. Mechanistically, the therapeutic effects of HSG4112 were dependent on Pon2 activity. Hepatic Pon2 activation by HSG4112 is closely associated with autophagy activation and antioxidant effects. Thus, HSG4112 is a pharmacological activator of Pon2 and a potential therapeutic for $\mathrm{NASH}$. 


\section{METHODS}

\section{Animals}

All animal experiments were performed according to the internationally accepted principles for the care and use of laboratory animals. The license for performing animal experiments was issued by the Danish Committee for animal research for Jacob Jelsing (2013-15-2934-00784). Male C57BL/6J mice aged 5 weeks (Janvier Labs, France) were housed in a controlled environment (circadian cycle, 12-h light/dark cycle (lights on at $3 \mathrm{AM}$ ); temperature, $21 \pm 2{ }^{\circ} \mathrm{C}$; humidity, $50 \% \pm 10 \%$ ). Individual mice were tagged with an implantable microchip (PetID Microchip, E-vet, Haderslev, Denmark). The animals had ad libitum access to tap water and a diet comprising $40 \%$ fat $(18 \%$ trans-fat), $40 \%$ carbohydrates (20\% fructose), and 2\% cholesterol (D09100301, Research Diets, USA). Mice were fed on this diet for 35-37 weeks to establish a severe NASH phenotype. The liver of all mice was biopsied before drug treatment. Fibrosis and steatosis stages in the liver biopsy were analyzed at baseline (fibrosis score $\geq 1$, steatosis score $\geq 2$ ). Individual animals were placed in separate cages. The total number of animals used for each experiment is indicated in the figures and table. An outline of the study design is shown in Figures 1 and 2.

\section{Baseline liver biopsy}

The liver was biopsied 4 weeks before drug treatment. Briefly, mice were pretreated with enrofloxacin (Bayer, Germany) one day before biopsy. Before the biopsy, mice were anesthetized with isoflurane in atmospheric air. A midline abdominal incision was introduced to expose the left lateral lobe and a cone-shaped liver biopsy sample (50-100 mg) was collected. The sample was fixed in $4 \%$ paraformaldehyde 
overnight and subjected to histological analysis. The cut surfaces were electrocoagulated using an electrosurgical unit (ERBE VIO 100C, ERBE, GA, USA). The liver was returned to the abdominal cavity, the abdominal wall was sutured, and the skin was stapled. Mice were intraperitoneally administered with carprofen (Pfizer, USA) (5-0.01 mL/kg bodyweight) at the time of surgery and on days 1 and 2 postsurgery to manage postoperative pain and infection. At week 3 post-biopsy, mice were randomized into various treatment groups based on bodyweight and liver fibrosis and steatosis scores.

\section{Drug treatment}

SGDs were prepared at Glaceum Inc. (Suwon, Republic of Korea) following the protocols mentioned in the patent US9783551B2. Mice were orally administered with SGDs at doses of 50 and $100 \mathrm{mg} / \mathrm{kg}$ bodyweight. One group of mice was subcutaneously administered with semaglutide (30 nmol/kg bodyweight) once daily. Drug treatment and diet were continued for 6 weeks (for experiments comparing the effects of HSG4112 and HSG4113) or 10 weeks (for experiments comparing the effects of semaglutide and HSG4112). Food intake was measured once daily for 2 weeks after the initiation of drug treatment. The blood samples were collected from the tail vein of mice under fasting conditions five days before the end of the experiment to determine the blood glucose and insulin levels. Additionally, a terminal blood sample was collected from the tail vein of non-fasting mice for plasma biochemical analysis. Mice were sacrificed by cardiac puncture under isoflurane anesthesia. The liver samples were excised, weighed, and processed for further analysis. 


\section{Bodyweight and body composition analysis}

Bodyweight was monitored daily during the treatment period. Whole-body fat and lean mass were analyzed during the 10 weeks of treatment using non-invasive EchoMRI scanning with EchoMRI-900 (EchoMRI, TX, USA). During the scanning procedure, mice were placed in a restrainer for 90-120 s. To analyze the body composition parameters at the end of the study period, the weights of the liver, epididymal white adipose tissue (EWAT), peri-renal white adipose tissue, and gastrocnemius muscle were determined.

\section{Plasma and tissue biochemical analyses}

Evaluation of Fgf21, Lep, and cytokines in the cardiac blood samples. The abdominal cavity of mice was opened under isoflurane anesthesia and the cardiac blood sample was collected using a syringe. The blood sample was transferred into a microvette/vacuette containing an anticoagulant, mixed five times by inversion, stored at $4^{\circ} \mathrm{C}$ until centrifugation. The plasma samples (supernatant) were transferred to new tubes and immediately frozen on dry ice and stored at $-80^{\circ} \mathrm{C}$ until use.

Evaluation of glucose, insulin, ALT, AST, ALP, and total cholesterol (TC) levels in the tail blood samples. Tail blood $(\leq 200 \mu \mathrm{L})$ was pressed into an open microvette $(200 \mu \mathrm{L})$ containing an appropriate anticoagulant. The blood samples were stored at $4^{\circ} \mathrm{C}$ until centrifugation. The plasma was separated, transferred to new tubes, and immediately frozen on dry ice and stored at $-80^{\circ} \mathrm{C}$ until use.

Evaluation of blood glucose and insulin levels. The blood samples were collected into heparinized glass capillary tubes and immediately suspended in glucose/lactate system solution buffer (EKF-Diagnostics, Germany). Next, the blood 
glucose levels were measured using a BIOSEN c-Line glucose meter (EKFDiagnostics), following the manufacturer's instructions. For analyzing insulin levels, the blood samples were collected in heparinized tubes and the plasma was separated. The plasma samples were stored at $-80^{\circ} \mathrm{C}$ until use. The insulin levels were measured using the MSD platform (Meso Scale Diagnostics), following the manufacturer's instructions.

Evaluation of TC, ALT, AST, and ALP. The blood samples were collected in heparinized tubes and the plasma was separated. The plasma samples were stored at $-80^{\circ} \mathrm{C}$ until use. The TC, ALT, AST, and ALP levels were measured using commercial kits (Roche Diagnostics) with the Cobas c501 autoanalyzer, following the manufacturer's instructions.

Evaluation of Fgf21, cytokines, and Lep. The blood samples were collected in ethylenediaminetetraacetic acid (EDTA)-coated tubes and the plasma was separated. The plasma samples were stored at $-80^{\circ} \mathrm{C}$ until use. The Fgf21, Tnfa, $\|10\| 5,, \| 6$, and Cxcl1 levels in the plasma were measured in duplicates using an enzyme-linked immunosorbent assay (ELISA) kit (BioVendor), following the manufacturer's instructions. The levels of leptin and insulin were measured using the MSD platform (Meso Scale Diagnostics), following the manufacturer's instructions.

Liver TC analysis. The liver samples were homogenized and heated twice at $90^{\circ} \mathrm{C}$ in $5 \% \mathrm{NP}-40$ to extract TC. The samples were centrifuged and the TC content in the supernatant was measured using commercial kits (Roche Diagnostics) with the Cobas c501 autoanalyzer, following the manufacturer's instructions.

\section{Liver histological and quantitative analyses}


Biopsy samples obtained at the baseline and the end of the study period (both from the left lateral lobe) were fixed overnight in $4 \%$ paraformaldehyde. The liver tissue was paraffin-embedded and sectioned ( $3 \mu \mathrm{m}$ thickness). The sections were stained with H\&E and anti-Lgals3, anti-Acta1, anti-Adgre1, anti-Map1lc3b, and anti-Becn1 antibodies, following the manufacturer's instructions. The samples were subjected to quantitative histomorphometry using Visiomorph ${ }^{\circledR}$ digital imaging software (Visiopharm, Hørsholm, Denmark) or NIH ImageJ software (http://rsbweb.nih.gov/ij/). The primary antibodies used in this study are listed in Supplementary Table 3. The fractional area of liver fat accumulation (macrosteatosis) was determined using HE-stained sections and expressed as a percentage of the total sectional area. The hepatic lipid droplet accumulation, which indicates hepatic steatosis, was confirmed using oil red $\mathrm{O}$ staining. Liver fibrosis was examined using picrosirius red staining. The Lgals3-positive and Acta1-positive areas were expressed as a percentage of the total parenchymal area by subtracting the corresponding fat area determined on adjacent HE-stained sections. Macrophage infiltration in the liver was assessed based on Adgre1 immunostaining and $\mathrm{Ccl} 2$ expression. Autophagy activation in the liver was determined based on Map1lc3b and Becn1 immunostaining. The staining intensities of Map1lc3b and Becn1 are represented as inverted median pixel values (IMPVs). Hepatic oxidative stress was assessed using the thiobarbituric acid reactive substance (TBARS) assay. To examine adiposity, the paraffin-embedded EWAT sections were subjected to H\&E staining. The average adipocyte size in EWAT was measured using morphometry with NIH ImageJ software. All histological assessments were performed by experienced histologists who were blinded to the experimental groups. Steatosis, lobular inflammation, hepatocyte 
ballooning, and fibrosis in the liver were scored before and after treatment using the NAS and fibrosis staging system.

\section{RNAseq and data analysis}

The liver samples after treatment (15 $\mathrm{mg}$ fresh tissue) and gastrocnemius muscle were subjected to RNAseq. The RNA quantity and quality were measured using Qubit ${ }^{\circledR}$ (Thermo Fisher Scientific, OR, USA) and a bioanalyzer with RNA 6000 Nano kit (Agilent, Waldbronn, Germany), respectively. The RNA sequence libraries were prepared using NeoPrep (Illumina, CA, USA) with Illumina TruSeq stranded mRNA Library kit for NeoPrep (Illumina) and sequenced on the NextSeq 500 (Illumina) with NSQ 500 hi-Output KT v2 (75 CTS, Illumina). The sequencing reads were aligned to the GRCm38 v84 Ensembl Mus musculus genome using STAR v.2.5.2a with default parameters. Differential gene expression analysis was performed with DEseq2. The difference in the expression of genes with a Benjamini-Hochberg-adjusted $p$-value of $\leq$ $0.05(5 \%$ false discovery rate (FDR)) was considered significant.

\section{Proteomics}

Protein extraction and digestion. Cryo-pulverized liver tissues (wet weight: 40$50 \mathrm{mg}$ ) were homogenized in $1 \mathrm{~mL}$ radioimmunoprecipitation assay (RIPA) lysis buffer (Pierce) (5\% sodium dodecyl sulfate (SDS), $0.1 \mathrm{M}$ Tris- $\mathrm{HCl}(\mathrm{pH} 7.6), 1 \times$ Halt protease inhibitor (Pierce), and 1× phosphatase inhibitor (PhosSTOP, Roche)). The lysates were centrifuged at $20,000 \mathrm{~g}$ for $10 \mathrm{~min}$ and the supernatant was transferred to a new tube. The protein concentration in the supernatant was measured using the bicinchoninic acid (BCA) protein assay kit (Pierce). Protein samples $(600 \mu \mathrm{g})$ were divided into two 
equal portions, and each portion was digested separately using an S-Trap method (Katelyn et al., 2018). Briefly, $300 \mu \mathrm{g}$ proteins were incubated with $20 \mathrm{mM}$ dithiothreitol (DTT; Sigma Aldrich) for $10 \mathrm{~min}$ at $95^{\circ} \mathrm{C}$ on a thermomixer (Thermo Fisher Scientific). The proteins were then alkylated with $50 \mathrm{mM}$ iodoacetamide (Sigma Aldrich) for $30 \mathrm{~min}$ in the dark. Before digestion, phosphoric acid at a final concentration of $1.2 \%$ and six volumes of binding buffer $(90 \%$ methanol; $100 \mathrm{mM}$ triethylammonium bicarbonate (TEAB); $\mathrm{pH}$ 7.1) were added to the sample and gently mixed. The protein solution was loaded to an S-Trap filter and centrifuged at $3000 \mathrm{rpm}$ for $1 \mathrm{~min}$. The flow-through was collected and reloaded onto a filter. This step was repeated thrice. The filter was washed thrice with $300 \mu \mathrm{L}$ of binding buffer. Digestion was performed with Lys-C (Pierce) for $1 \mathrm{~h}$ and trypsin (Pierce) overnight (enzyme-to-protein ratio $=1: 50$ ). The peptides were eluted twice using the following three buffers (200 $\mu \mathrm{L}$ each): $50 \mathrm{mM}$ TEAB, $0.2 \%$ formic acid in $\mathrm{H}_{2} \mathrm{O}$, and $50 \%$ acetonitrile and $0.2 \%$ formic acid in $\mathrm{H}_{2} \mathrm{O}$.

Tandem mass tag (TMT)-16 labeling and peptide fractionation. The eluted peptides $(400 \mu \mathrm{g})$ from each sample were labeled with 16-plex TMT reagents (Thermo Fisher Scientific, Germany), following the manufacturer's instructions. The peptides were solubilized in $100 \mu \mathrm{L}$ of $100 \mathrm{mM}$ TEAB $(\mathrm{pH}$ 8.5) solution and incubated with labeling reagent in $20 \mu \mathrm{L}$ of acetonitrile for $1 \mathrm{~h}$ with shaking. To quench the unreacted TMT reagents, $8 \mu \mathrm{L}$ of $5 \%$ hydroxylamine was added. Differentially labeled peptides were then mixed $(16 \times 400 \mu \mathrm{g})$ and dried using a vacuum centrifuge. The quenched, combined sample was desalted using a C18 column (Harvard apparatus). The pooled TMT-labeled peptide sample was fractionated using basic $\mathrm{pH}$ reverse-phase liquid chromatography with an Agilent 1260 Infinity HPLC system (Agilent, CA, USA). The chromatography conditions were as follows: column, Xbridge C18 analytical column 
(4.6 $\mathrm{mm} \times 250 \mathrm{~mm})$; solvent $\mathrm{A}, 10 \mathrm{mM}$ ammonium formate in water $(\mathrm{pH} \mathrm{9.5);} \mathrm{solvent} \mathrm{B}$, $10 \mathrm{mM}$ ammonium formate in $90 \%$ acetonitrile ( $\mathrm{pH}$ 9.5); gradient time, $105 \mathrm{~min}$; flow rate, $500 \mathrm{~mL} / \mathrm{min}$. The gradient conditions were follows: $0 \%$ solvent $\mathrm{B}$ for $10 \mathrm{~min} ; 0 \%-$ $13 \%$ solvent B for $10 \mathrm{~min} ; 13 \%-40 \%$ solvent $B$ for $60 \mathrm{~min} ; 40 \%-70 \%$ solvent $B$ for 15 min; $70 \%$ solvent $B$ for $10 \mathrm{~min} ; 70 \%-0 \%$ solvent $B$ for $10 \mathrm{~min}$. In total, 96 fractions were collected every minute from $15 \mathrm{~min}$ to $110 \mathrm{~min}$. The fractions were pooled into 24 non-contiguously concatenated peptide fractions. The resultant 24 fractions were dried and stored at $-80^{\circ} \mathrm{C}$.

Phosphopeptide enrichment. The phosphopeptides from 12 fractions obtained by combining sequential fractions of the 24 fractions (e.g. F1 and F2 or F3 and F4) were enriched using the immobilized metal affinity chromatography (IMAC). IMAC beads were prepared from Ni-nitrilotriacetic acid (NTA) magnetic agarose beads. NiNTA beads $(500 \mathrm{~mL})$ were washed thrice with water. The beads were then incubated with $100 \mathrm{mM}$ EDTA ( $\mathrm{pH}$ 8.0) for 30 min with end-over-end rotation to remove nickel ions. The EDTA solution was removed and the beads were washed thrice with water. The NTA beads were treated with $10 \mathrm{mM}$ of aqueous $\mathrm{FeCl}_{3}$ solution for 30 min with endover-end rotation. Iron-chelated IMAC beads were washed thrice with water. Ni-NTA agarose beads were used to prepare $\mathrm{Fe}^{3+}$-NTA agarose beads. Approximately $300 \mu \mathrm{g}$ of peptides from each phosphoproteome fraction were reconstituted in $500 \mu \mathrm{L}$ of $80 \%$ MeCN/0.1\% trifluoroacetic acid (TFA). The solution was incubated with IMAC beads for $30 \mathrm{~min}$ on a shaker at room temperature. The samples were briefly centrifuged using a tabletop centrifuge. The clarified peptide flow-throughs were separated from the beads and the beads were reconstituted in $200 \mathrm{~mL}$ IMAC binding/wash buffer ( $80 \mathrm{MeCN} / 0.1 \%$ TFA) and loaded onto equilibrated Empore C18 silica-packed stage tips (3M, 2315). 
The samples were then washed twice with $50 \mu \mathrm{L}$ of IMAC binding/wash buffer and once with $50 \mu \mathrm{L}$ of $1 \% \mathrm{FA}$ and eluted thrice from the IMAC beads to the stage tips with $70 \mu \mathrm{L}$ of $500 \mathrm{mM}$ dibasic sodium phosphate (pH 7.0, Sigma Aldrich, S9763). The stage tips were then washed once with $100 \mathrm{~mL} 1 \%$ FA. Phosphopeptides were eluted from the stage tips with $60 \mu \mathrm{L}$ of $50 \% \mathrm{MeCN} / 0.1 \% \mathrm{FA}$.

LC-ESI-MS/MS analysis of the proteome. All peptide samples were separated using an ultra-performance liquid chromatography system equipped with analytical columns $(75 \mu \mathrm{m} \times 50 \mathrm{~cm}, \mathrm{C} 18,3 \mu \mathrm{m}, 100 \AA)$ and trap columns $(75 \mu \mathrm{m} \times 2 \mathrm{~cm}, \mathrm{C} 18,3$ $\mu \mathrm{m}, 100 \AA$ ). The mobile phases were as follows: solvent $A, 0.1 \%$ formic acid in water; solvent $\mathrm{B}, 0.1 \%$ formic acid in $90 \%$ acetonitrile. The solvent gradient conditions for global proteome profiling analysis were as follows: $2 \%$ solvent $B$ for $8 \mathrm{~min}, 2 \%-10 \%$ solvent B for $3 \mathrm{~min}, 10 \%-25 \%$ solvent $B$ for $113 \mathrm{~min}, 25 \%-40 \%$ solvent $B$ for $20 \mathrm{~min}$, $70 \%$ solvent $\mathrm{B}$ for $4 \mathrm{~min}$, and $2 \%$ solvent $\mathrm{B}$ for $20 \mathrm{~min}$. Meanwhile, the gradient conditions for phosphoproteome analysis were as follows: $2 \%$ solvent $B$ for $8 \mathrm{~min}, 2 \%-$ $10 \%$ solvent $B$ for $3 \mathrm{~min}, 10 \%-20 \%$ solvent B for $123 \mathrm{~min}, 20 \%-30 \%$ solvent $B$ for 20 min, $70 \%$ solvent $B$ for 4 min, and $2 \%$ solvent B for 20 min. For global peptide analysis, $2 \mu \mathrm{g}$ of peptides from each of the 24 fractions were individually analyzed. All enriched peptides from each of the 12 fractions were injected for phosphopeptide analysis. The eluted peptides from LC were analyzed with a $Q$ exactive plus orbitrap mass spectrometer (Thermo Fisher Scientific) equipped with an easy-spray nano source. Data acquisition was performed using Xcalibur Q Exactive v2.1 software in positive ion mode at a spray voltage of $2.0 \mathrm{kV}$. MS1 spectra were captured under the following conditions: resolution, 70,000; AGC target, 1e6; mass range, 400-1800 m/z; mode cycle time, 2 s. MS2 spectra were captured under the following conditions: resolution, 
35,000; AGC target, 2e5; isolation window, $2.0 \mathrm{~m} / \mathrm{z}$; maximum injection time, $100 \mathrm{~ms}$; HCD collision energy, $32 \%$. The peptide mode was selected for monoisotopic peak determination. Charge state screening was enabled to only include precursor charge states 2-6 with an intensity threshold of $5 \mathrm{e} 4$. Peptides that triggered MS/MS scans were dynamically excluded from further MS/MS scans for $30 \mathrm{~s}$ with a mass tolerance of $10 \mathrm{ppm}( \pm)$.

Protein and phosphopeptide identification. The acquired raw files were processed using Proteome Discoverer v.2.4 (Thermo Fisher Scientific) software environment with the built-in Sequest HT search engine for the identification of peptides and phosphopeptides. The data were searched using a target-decoy approach against Uniprot Human (Jan 2021, 20,452 entries) reference proteome (FASTA file) (FDR < 1\%) at the level of proteins, peptides, and modifications using minor changes to the default settings as follows: oxidized methionine (M); in case of phosphopeptides, search phospho $(\mathrm{S}, \mathrm{T}, \mathrm{Y})$ was selected as variable modifications, while carbamidomethyl $(\mathrm{C})$ selected as fixed modification. A maximum of 2 missed cleavages and a minimum peptide length of seven amino acids were allowed. Enzyme specificity was set to trypsin. An initial precursor mass deviation up to $10 \mathrm{ppm}$ and a fragment mass deviation up to $0.06 \mathrm{Da}$ were allowed. To quantify each reporter ion in the sample, 'reporter ion quantifier' with TMT 16-plex was used. For highly confident quantifications of protein, the protein ratios were calculated from two or more unique quantitative peptides in each replicate. All reporter ion intensities were transformed to log2 values. Proteins that did not display all values in at least one group were filtered out. 
Proteomic data analysis. For global proteome analysis, the TMT intensities of the peptides were normalized using the quantile normalization method. Differentially expressed proteins (DEPs) were identified using an integrative statistical method. Briefly, $\log 2$ (intensity) of each protein was applied to its abundance for all replicates. The fold change and adjusted p-value were calculated using Student's t-test. Finally, DEPs that had a combined fold change value of 1.5 and a $p$-value $<0.05$ were selected. For phosphoproteome analysis, the same normalization and statistical method described above were used. Differentially phosphorylated peptides (DPPs) that were uniquely assigned to the protein were selected. To explore the signal pathway in which the DEPs and DPPs are enriched, Kyoto Encyclopedia of Genes and Genomes (KEGG) enrichment and Reactome pathway analyses were performed using the g:Profiler. To reconstruct drug-related network modeling, protein-protein interactions (PPIs) were obtained from the STRING database. Network models were developed for a list of the selected proteins for network analysis based on the collected PPIs. In the network models, the nodes were arranged based on the KEGG and Reactome Pathway database analyses.

\section{Lipidomics}

Lipid extraction. Liver samples were weighed and individually cryo-pulverized using a Cryoprep device (CP02, Covaris). The lyophilized samples from each tissue were aliquoted to separate tubes (50 $\mathrm{mg}$ for proteomic analysis and $20 \mathrm{mg}$ for lipidomic analysis) and stored at $-80^{\circ} \mathrm{C}$. For lipid analysis of liver tissues, two-step lipid extraction method was performed. To perform the neutral extraction, liver tissues were thawed on ice and incubated with internal standards $(10 \mu \mathrm{L})$ and methanol/chloroform 
$(2: 1(v / v) ; 990 \mu \mathrm{L})$. The samples were vortexed for $60 \mathrm{~s}$. The standard mix SPLASH Lipidomix Mass Spec Standard j 330707 supplemented with LPG (14:0), LPI (13:0), LPA (14:0), Cer (d18:1-12:0), So (d17:1), Sa (d17:0), CerP (d18:1-12:0), So1P (d17:1), Sa1P (d17:0), and FFA (20:4-d8) was used. The sample was incubated for 10 min on ice and centrifuged $15,000 \mathrm{~g}$ and $4^{\circ} \mathrm{C}$ for $2 \mathrm{~min}$. Next, $950 \mu \mathrm{L}$ of supernatant was transferred to a new tube. To perform the acidic extraction, the remaining tissue was incubated with $750 \mu \mathrm{L}$ of chloroform/methanol/37\% $\mathrm{HCl}(40: 80: 1 \mathrm{v} / \mathrm{v} / \mathrm{v})$ mixture for 15 min at room temperature. Next, $250 \mu \mathrm{L}$ of cold chloroform and $450 \mu \mathrm{L}$ of cold $0.1 \mathrm{~N} \mathrm{HCl}$ were added to the sample. The samples were vortexed for $1 \mathrm{~min}$ and centrifuged at $7000 \mathrm{~g}$ and $4^{\circ} \mathrm{C}$ for $2 \mathrm{~min}$. The bottom organic phase was collected and pooled with a prior extract and dried using a SpeedVac concentrator.

Lipid measurement using LC-MS. HPLC-ESI-MS/MS analyses were performed using a 1200 series HPLC system (Agilent Technologies, DE, USA) coupled to 6490 Accurate-Mass Triple Quadrupole Mass Spectrometer (Agilent Technologies). The LCMS/MS conditions were as follows: column, Hypersil GOLD column $(2.1 \times 100 \mathrm{~mm}$ ID; $1.9 \mu \mathrm{m}$, Thermo Fisher Scientific); solvent A (acetonitrile/methanol/water (19:19:2 v/v/v) $+20 \mathrm{mmol} / \mathrm{L}$ ammonium formate $+0.1 \%(\mathrm{v} / \mathrm{v})$ formic acid); solvent $\mathrm{B}$, (2-propanol +20 $\mathrm{mmol} / \mathrm{L}$ ammonium formate $+0.1 \%(\mathrm{v} / \mathrm{v})$ formic acid); flow rate, $250 \mu \mathrm{L} / \mathrm{min}$; total run time, $33 \mathrm{~min}$; sample injection volume, $5 \mu \mathrm{L}$. The gradient elution program was as follows: $0-5 \mathrm{~min}$, solvent B $3 \%$; 5-18 min, solvent B $5-30 \%$; $18-24 \mathrm{~min}$, solvent B 30\%-90\%; 24-28 min, solvent B 90\%; 28-29 min, 90\%-3\%; and 29-33 min, solvent B $3 \%$. The parameters of operating source conditions were as follows: capillary voltage in positive mode, $3500 \mathrm{~V}$; capillary voltage in negative mode, $3000 \mathrm{~V}$; sheath gas flow rate, $11 \mathrm{~L} / \mathrm{min}$ (UHP nitrogen); sheath gas temperature, $200^{\circ} \mathrm{C}$; drying gas flow rate, 15 
$\mathrm{L} / \mathrm{min}$; drying gas temperature, $150^{\circ} \mathrm{C}$; nebulizer gas pressure, $25 \mathrm{psi}$. The optimal dMRM conditions were used to analyze various lipid species.

Lipidomics data analysis. Agilent Mass Hunter Workstation Data Acquisition software was used to process the LC/MS data. Qualitative Analysis B.06.00 software (Agilent Technologies) was used to export the $\mathrm{m} / \mathrm{z}$ of precursor and product ions and retention time of target lipids in the multiple reaction monitoring data. The area of the assigned peak in the raw data was calculated using an in-house database constructed using Skyline software package (MacCoss Laboratory, University of Washington, WA, USA). Lipid abundance was normalized to tissue weight and internal standard peak area. MetaboAnalyst Web site (https://metaboanalyst.ca) was used to perform PCA and projection to latent structure discriminant analysis (PLS-DA). Unweighted correlation network graphs were generated based on Spearman's correlation. All nodes with $\mathrm{P}$ values less than 0.05 were used in SPSS version 21.0 (IBM Corp, NY, USA). Heatmaps were generated using the Morpheus Web site (https://software.broadinstitute.org/morpheus).

\section{Cell culture and generation of stable Pon2 knockdown cells}

L02 cells (an immortalized normal liver cell line) were a kind gift from Dr. KH Lee (Korea Institute of Radiological and Medical Sciences). The cells were cultured in Dulbecco's modified Eagle's medium (DMEM) (Welgene, LM001-05) supplemented with $10 \%$ fetal bovine serum and $100 \mathrm{U} / \mathrm{mL}$ of penicillin and streptomycin at $37^{\circ} \mathrm{C}$ in a $5 \% \mathrm{CO}_{2}$ incubator.

Stable Pon2 knockdown (KD) L02 cells were generated by transducing the cells with recombinant lentivirus harboring short hairpin RNA against Pon2 (shPON2; 
Gencopoeia, LPP-HSH013480-LVRU6P). Pon2 KD cells were cultured in DMEM supplemented with puromycin (500 ng/mL) (Life Technologies, A11138-03). The KD of PON2 was confirmed using immunoblotting with the anti-Pon2 antibodies.

Cells stably expressing mt-Keima were generated by transducing the cells with recombinant lentivirus containing mt-Keima cDNA for $48 \mathrm{~h}$. The recombinant cells were selected with puromycin $(500 \mathrm{ng} / \mathrm{mL})$. The packaging vectors pSPAX2 and pMD2.0G were co-transfected with pmt-Keima into HEK293T cells for $72 \mathrm{~h}$. The cell supernatant was filtered through a $0.22-\mu \mathrm{m}$ membrane filter. The virus was concentrated by subjecting the samples to ultra-centrifugation at $20000 \mathrm{rpm}$. The supernatant was discarded and the viruses were suspended in an appropriate amount of PBS and stored at $-80^{\circ} \mathrm{C}$ until use.

Bovine serum albubim (BSA)-conjugated oleic acid (OA, O3008) and PA (P0500) were purchased from Sigma Aldrich (St. Louis, MO). To prepare the BSA-conjugated PA solution, $100 \mathrm{mM}$ PA solution was prepared in $0.1 \mathrm{mM} \mathrm{NaOH}$ and the solution was heated at $70^{\circ} \mathrm{C}$. PA solution was incubated with $10 \% \mathrm{BSA}$ at $55^{\circ} \mathrm{C}$ for 30 min to obtain $5 \mathrm{mM}$ PA $/ 1 \%$ BSA. The solution was then cooled to $25^{\circ} \mathrm{C}$, filter-sterilized, and stored at $-20^{\circ} \mathrm{C}$ until use. The cells were incubated with culture medium containing $400 \mu \mathrm{M}$ FFA (150 $\mu \mathrm{M}$ BSA-conjugated $\mathrm{OA}$ and $250 \mu \mathrm{M}$ BSA-conjugated PA) in the presence or absence of $3 \mu \mathrm{M} \mathrm{HSG} 4112$.

\section{Lipid accumulation assay}

Cells were plated in six-well plates and incubated with $400 \mu \mathrm{M}$ FFA in the presence or absence of $3 \mu \mathrm{M}$ HSG4112 for $72 \mathrm{~h}$. The cells fixed with $4 \%$ paraformaldehyde for $10 \mathrm{~min}$ were stained with oil red O solution (Sigma Aldrich, 
01391) for $15 \mathrm{~min}$ at room temperature. After washing once with $60 \%$ isopropanol, the cells were rinsed with distilled water. The images were captured using an inverted microscope (Carl Zeiss Axioimager M2 fluorescence microscopy). The signal intensity was analyzed using the NIH ImageJ software.

\section{Autophagy flux analysis}

Immunoblotting analysis was performed to analyze the endogenous LC3B-II/I and Sqstm1/p62 expression levels with anti-Map1lc3b and anti-Sqstm1 antibodies, respectively, following the manufacturer's instructions (Supplemental Table 3). The cells were plated in six-well plates and incubated with $400 \mu \mathrm{M}$ FFA in the presence or absence of $3 \mu \mathrm{M}$ HSG4112 for the indicated durations. Next, the cells were lysed with SDS lysis buffer supplemented with a protease inhibitor cocktail (Thermo Fisher Scientific, 78441). The proteins were subjected to immunoblotting. The intensity of each protein signal was normalized to that of Actb. The control value was set to 1.0 and the protein intensity was represented relative to that of the control.

To monitor the autophagy level, LC3B puncta in cells transfected with mRFP-GFP tandem fluorescent-tagged LC3B (tfLC3B) were examined using immunofluorescence microscopy. The cells cultured on glass coverslips were transfected with mRFP-GFP tfLC3B plasmid. At $16 \mathrm{~h}$ post-transfection, the cells were treated with $400 \mu \mathrm{M}$ FFA and $3 \mu \mathrm{M}$ HSG4112 in the presence or absence of bafilomycin A1 (Invivogen, tlrl-baf1). Cellular localization of LC3B was observed using a Carl Zeiss Confocal LSM710 Meta microscope and the images were processed with the software supplied by the manufacturer (Carl Zeiss, Seoul, Republic of Korea) and analyzed with NIH ImageJ software. Cells containing three or more mRFP-LC3B puncta were defined as 
autophagy-positive cells. The percentage of autophagy-positive cells relative to the total number of mRFP-positive cells was calculated. At least 100 mRFP-positive cells per sample were counted in at least three independent experiments. Cells stained with both RFP and GFP were defined as autophagosome-positive, while those stained with RFP only were defined as autolysosome-positive. The number of fluorescent LC3B puncta was determined by counting more than 100 cells with triplicates.

\section{Mitophagy measurement}

Endogenous Prkn, Pink1, Bnip, Bnip3I, Map1lc3b, and Sqstm1 expression levels were analyzed using immunoblotting analysis with the respective antibodies, following the manufacturer's instructions (Supplemental Table 3). The cells were plated into sixwell plates and incubated with $125 \mu \mathrm{M}$ PA in the presence or absence of $3 \mu \mathrm{M}$ HSG4112 for $24 \mathrm{~h}$. Next, the cells were lysed with SDS lysis buffer supplemented with a protease inhibitor cocktail (Thermo Fisher Scientific, 78441). Immunoblotting was performed as described above.

To monitor the mitophagy level, an mt-Keima-based mitophagy assay was performed using immunofluorescence microscopy. The cells expressing mt-Keima were plated onto a confocal dish, incubated with $125 \mu \mathrm{M}$ PA in the presence or absence of 3 $\mu \mathrm{M}$ HSG4112 for $24 \mathrm{~h}$, and analyzed using confocal microscopy. Mt-Keima protein was excited at $458 \mathrm{~m}$ (neutral, pseudo-colored in green) and $561 \mathrm{~nm}$ (acidic, pseudocolored in red) and detected through the same emission filter (570-695 nm). Laser power was set up at the lowest output to enable the clear visualization of the mt-Keima signal and individualized for each experimental condition. Imaging settings were maintained with the same parameters for comparison between different experimental 
conditions. Additionally, the images were acquired using a Carl Zeiss Confocal LSM710 Meta microscope (Carl Zeiss, Seoul, Republic of Korea). The fluorescence intensity was quantified using the $\mathrm{NIH}$ ImageJ software.

\section{Measurement of mitochondrial superoxide}

Cells were plated in six-well plates and incubated with $125 \mu \mathrm{M}$ PA in the presence or absence of $3 \mu \mathrm{M}$ HSG4112 for $24 \mathrm{~h}$. Next, the cells were pulsed with $2.5 \mu \mathrm{M}$ MitoSOX red mitochondrial superoxide indicator (Thermo Fisher Scientific, M36008) and $5 \mu \mathrm{M}$ Hoechst 33342 (Thermo Fisher Scientific, H1399) for 30 min and subjected to live cell imaging at $37^{\circ} \mathrm{C}$. After washing, the cells were observed under a confocal microscope in a chamber heated to $37^{\circ} \mathrm{C}$ at $5 \% \mathrm{CO}_{2}$. Images were acquired using a Carl Zeiss Confocal LSM710 Meta microscope (Carl Zeiss, Seoul, Republic of Korea). The fluorescence intensity was quantified using the NIH ImageJ software.

\section{Lipid peroxidation assay}

Lipid peroxidation in the liver tissue and cells was determined by measuring the contents of malondialdehyde (MDA) resulting from the thiobarbituric acid reaction. The cells were plated in six-well plates and incubated with $125 \mu \mathrm{M}$ PA in the presence or absence of $3 \mu \mathrm{M}$ HSG4112 for $24 \mathrm{~h}$. Next, the cells were trypsinized and centrifuged. The cell pellets were washed with PBS and stored at $-80^{\circ} \mathrm{C}$ until use. The MDA concentration was determined using the TBARS parameter assay kit (R\&D systems, KGE013), following the manufacturer's instructions.

For performing the 4-hydroxynonenal (4-HNE) assay, the cells were lysed using RIPA buffer (Cell Signaling Technology) supplemented with a protease inhibitor cocktail 
(Thermo Fisher Scientific, 78441). The protein concentration was determined using the BCA protein assay kit (Thermo Fisher Scientific, 23225). The 4-HNE concentration was determined using the 4-HNE ELISA kit (BioVision, E4645-100), following the manufacturer's instructions.

\section{Measurement of oxygen consumption rate (OCR)}

OCR in human liver cells was measured using XFp Extracellular Flux Analyzers (Agilent Seahorse Biosciences). The cells were plated into XFp cell culture mini plates for $24 \mathrm{~h}$. The culture medium was replaced with XF-Base medium (non-buffered Roswell Park Memorial Institute-1640 medium containing $2 \mathrm{mM}$ L-glutamine, $1 \mathrm{mM}$ sodium pyruvate, and $10 \mathrm{mM}$ glucose, $\mathrm{pH} 7.4$ ) for $30 \mathrm{~min}$. Next, the cells were incubated with HSG4112 $(3 \mu \mathrm{M})$ for $15 \mathrm{~min}$, followed by incubation with $250 \mu \mathrm{M}$ palmitate-BSA or BSA (control). Analysis was performed using the XF assay with the cell mito stress test kit (Agilent Seahorse Biosciences). Three measurements were assessed under basal conditions and after the addition of $2 \mu \mathrm{M}$ oligomycin, $0.5 \mu \mathrm{M}$ carbonyl cyanide-p-trifluoromethoxyphenylhydrazon $\quad$ (FCCP), and $1 \mu \mathrm{M}$ rotenone/antimycin. The cellular protein levels were evaluated using the BCA protein assay kit (Thermo Fisher Scientific, 23225). OCR values were normalized to the protein concentration.

To determine FAO, cells were cultured in XFp cell culture mini plates for $24 \mathrm{~h}$. The culture medium was replaced with substrate limited medium for $30 \mathrm{~min}$. Next, the cells were treated with etomoxir (ETO; $40 \mu \mathrm{M}$ final concentration, Agilent Seahorse Biosciences) in the presence or absence of HSG4112 (3 $\mu \mathrm{M})$ for $15 \mathrm{~min}$, followed by incubation with $250 \mu \mathrm{M}$ palmitate-BSA or BSA (control). Analysis was performed using 
the XF assay with the cell mito stress test kit (Agilent Seahorse Biosciences). Three measurements were assessed under basal conditions and after the addition of $2 \mu \mathrm{M}$ oligomycin, $0.5 \mu \mathrm{M} F C C P$, and $1 \mu \mathrm{M}$ rotenone/antimycin. The cellular protein levels were evaluated using the BCA protein assay kit (Thermo Fisher Scientific, 23225). The OCR values were normalized to the protein concentration.

\section{Intracellular localization of HSG4112}

Cells were cultured on glass coverslips and pulsed in the presence of propargylHSG4112 $(3 \mu \mathrm{M})$ for $5 \mathrm{~h}$. After labeling, the cells were washed thrice with PBS and incubated in serum-free media with $100 \mathrm{nM}$ MitoTracker Red (Invitrogen, M7512) for 30 min at $37^{\circ} \mathrm{C}$ in a $5 \% \quad \mathrm{CO}_{2}$ incubator. The cells were then fixed with $4 \%$ paraformaldehyde in PBS for $10 \mathrm{~min}$ and stained with $10 \mu \mathrm{M}$ Alexa488-azide (Invitrogen, A10266) for 30 min using the Click-iT cell reaction buffer kit (Invitrogen, C10269), following the manufacturer's instructions. Next, the cells were washed thrice with PBS. The images were acquired using a Carl Zeiss Confocal LSM710 Meta microscope (Carl Zeiss, Seoul, Republic of Korea). The data were analyzed using NIH ImageJ software.

\section{Quantification of Pon2 enzyme activity}

Intracellular Pon2 activity. Cells were plated into six-well plates and incubated with $125 \mu \mathrm{M}$ PA in the presence or absence of $3 \mu \mathrm{M}$ HSG4112 for $24 \mathrm{~h}$. Next, the cells were trypsinized and centrifuged. The cell pellets were washed with PBS and stored at $-80^{\circ} \mathrm{C}$ until use. The frozen cell pellets were incubated with $25 \mathrm{mM}$ Tris buffer $(\mathrm{pH} 7.4)$ 
containing $0.05 \% \mathrm{n}$-dodecyl- $\beta$-D-maltoside (Merck, D4641) and $1 \mathrm{mM} \mathrm{CaCl}$, and lysed by subjecting them to three freeze-thaw cycles.

For evaluating the Pon2 esterase activity, p-nitrophenyl acetate (pNPA) hydrolysis was determined using a SpectraMax Plus384 microplate reader (Agilent Technologies, Seoul, Republic of Korea). The cell lysates were transferred to a 96-well plate and reactions $(0.2 \mathrm{~mL}$ final mixture volume) were initiated by adding $1 \mathrm{mM}$ pNPA in Pon2 activity assay buffer $(50 \mathrm{mM}$ Tris $(\mathrm{pH} 8.0)$ with $1 \mathrm{mM} \mathrm{CaCl}$ ). The increase in absorbance at $412 \mathrm{~nm}$ resulting from the release of $\mathrm{p}$-nitrophenol was monitored.

For evaluating the Pon2 lactonase activity, the enzymatic hydrolysis of the thioalkyl-substituted lactones was determined. The cell lysates were transferred to a 96-well plate and reactions were initiated by adding $1 \mathrm{mM}$ 5-thiobutyl butyrolactone (TBBL) and $1 \mathrm{mM} \mathrm{5',5-dithiobis} \mathrm{(2-nitrobenzoic} \mathrm{acid)} \mathrm{(DTNB)} \mathrm{(Sigma} \mathrm{Aldrich,} \mathrm{D8130)} \mathrm{in}$ Pon2 activity assay buffer. The enzymatic hydrolysis was monitored by examining the absorbance of the reaction mixture at $420 \mathrm{~nm}$.

Recombinant PON2 activity. For performing the oxidized linoleic acid (Ox-LA)mediated Pon2 inhibition assay, purified recombinant Pon2 protein $(10 \mu \mathrm{M})$ was incubated with or without HSG4112 (10 $\mu \mathrm{M})$ in the Pon2 activity assay buffer in a 96well plate for $10 \mathrm{~min}$ at room temperature. To inhibit Pon2 activity, the samples were incubated with Ox-LA $(100 \mu \mathrm{M})$ for $10 \mathrm{~min}$ at RT. For evaluating the esterase activity, the reactions were initiated by adding $1 \mathrm{mM}$ pNPA in Pon2 activity assay buffer. The enzymatic hydrolysis was monitored by measuring the absorbance of the reaction mixture at $412 \mathrm{~nm}$. For evaluating the lactonase activity, the reactions were initiated by adding $1 \mathrm{mM}$ TBBL and $1 \mathrm{mM}$ DTNB in Pon2 activity assay buffer. The enzymatic 
hydrolysis was monitored by measuring the absorbance of the reaction mixture at 420 $\mathrm{nm}$.

\section{Chemical-protein interactome analysis for identifying HSG4112 target proteins}

Preparation of protein samples. Biotin-conjugated HSG4112 was synthesized by incubating $20 \mu \mathrm{M}$ propargyl-HSG4112 with $10 \mu \mathrm{M}$ biotin-azide (Invitrogen, B10184) using the Click-iT cell reaction buffer kit (Invitrogen, C10269) for $30 \mathrm{~min}$, following the manufacturer's instructions. Biotin-conjugated HSG4112 was incubated with Dynabbeads $^{\mathrm{TM}} \mathrm{M}-270$ streptavidin (Invitrogen, 65305) at $4^{\circ} \mathrm{C}$ for $1 \mathrm{~h}$. The HSG4112bead complex was washed thrice with PBS. The cell lysates were incubated with the HSG4112-bead complex at $4^{\circ} \mathrm{C}$ overnight. The beads were washed thrice with PBS and the bound proteins were eluted with SDS-PAGE sample buffer and stored at $-80^{\circ} \mathrm{C}$ until use.

Interactome analysis of target proteins of HSG4112. To identify the HSG4112binding proteins, the bound proteins were digested using the previously reported FASP protocol (Jung, et al, 2018). Briefly, the proteins were reduced in SDS lysis buffer (4\% (w/v) SDS; $0.1 \mathrm{M} \mathrm{Tris} / \mathrm{HCl}(\mathrm{pH} 7.6) ; 0.1 \mathrm{M} \mathrm{DTT})$ at $37^{\circ} \mathrm{C}$ for $45 \mathrm{~min}$ and boiled at $95^{\circ} \mathrm{C}$ for $10 \mathrm{~min}$. The solution was concentrated using a $30 \mathrm{k}$ membrane filter (Microcon devices, YM-30, Millipore, MA). The buffer was replaced with $0.2 \mathrm{~mL}$ UA solution (8 M urea in $0.1 \mathrm{M}$ Tris $/ \mathrm{HCl}(\mathrm{pH} 8.5))$. The concentrates were mixed with $0.1 \mathrm{~mL}$ of $50 \mathrm{mM}$ indole-3-acetic acid (IAA) in UA solution and incubated in the dark at room temperature for $30 \mathrm{~min}$. The samples were centrifuged, washed with $0.2 \mathrm{~mL}$ of $50 \mathrm{mM}$ TEAB, and centrifuged thrice at $14,000 \mathrm{~g}$ for $30 \mathrm{~min}$. Next, $500 \mathrm{ng}$ of trypsin prepared in $0.1 \mathrm{~mL}$ of 
$100 \mathrm{mM}$ TEAB (with the enzyme-to-protein ratio of 1:50) was added to the filter and the samples were incubated at $37^{\circ} \mathrm{C}$ overnight. The peptides were collected by centrifuging the filter units at $14,000 \mathrm{~g}$ for $30 \mathrm{~min}$. TEAB $(50 \mu \mathrm{L}, 50 \mathrm{mM})$ was added to the filter and the samples were centrifuged twice at $14,000 \mathrm{~g}$ for $20 \mathrm{~min}$. Analytical columns $(75 \mu \mathrm{m}$ $\times 50 \mathrm{~cm}, \mathrm{C} 18,3 \mu \mathrm{m}, 100 \AA)$ and trap columns $(75 \mu \mathrm{m} \times 2 \mathrm{~cm}, \mathrm{C} 18,3 \mu \mathrm{m}, 100 \AA)$ were used to separate the peptide samples. The solvents $A$ and $B$ were $0.1 \%$ formic acid in water and $0.1 \%$ formic acid in acetonitrile, respectively. The proteome profile analysis conditions were as follows: $2 \%-30 \%$ solvent B for 60 min, $25 \%-90 \%$ solvent B for 2 min, $90 \%$ solvent B for $8 \mathrm{~min}$, and $2 \%$ solvent B for 20 min. The eluted peptides from LC were subjected to mass spectrometry using a $Q$ Exactive HF-X Mass Spectrometer (Thermo Fisher Scientific, Bremen, Germany). Protein identification was performed using Proteome Discoverer 2.4 (Thermo Fisher Scientific) software and Uniprot Human (Jan 2021, 20,452 entries) database. Cellular components and biological processes in which each protein was enriched were analyzed using GO with g;Profiler.

\section{Evaluation of HSG4112-Pon2 interaction using the competitive binding assay}

To confirm the interaction between HSG4112 and Pon2, the HSG4112-bead complex was prepared as described above and incubated with cell lysates at $4^{\circ} \mathrm{C}$ overnight. The beads were washed thrice with PBS, eluted with SDS-PAGE sample buffer, and subjected to immunoblotting with the anti-Pon2 antibodies.

For performing the competitive binding assay, cell lysates were incubated with free HSG4112 at the indicated concentrations $(0-5 \mu \mathrm{M})$ for $5 \mathrm{~h}$ at $4^{\circ} \mathrm{C}$. Next, the HSG4112- 
bead complex was incubated with the free HSG4112-treated cell lysates at $4^{\circ} \mathrm{C}$ overnight. The beads were washed thrice with PBS and the bounded proteins were eluted with SDS-PAGE sample buffer. The samples were then subjected to immunoblotting with the anti-Pon2 antibodies.

\section{Immunohistochemical analysis of liver tissues}

The sections were deparaffinized with xylene and dehydrated with ethanol. Next, the sections were subjected to antigen retrieval and incubated with blocking solution to prevent nonspecific antibody binding. The sections were then probed with primary antibodies at $4^{\circ} \mathrm{C}$ overnight, following the manufacturer's instructions (Supplemental Table 3). After counterstaining with hematoxylin QS (Vector Laboratories, $\mathrm{H}-3404$ ), the sections were dehydrated and mounted. The staining intensity of each protein was measured using the NIH ImageJ software with the IHC Profiler plugin (http://rsb.info.nih.gov/ij/). The Map1lc3b and Becn1 intensities are represented as IMPVs.

\section{Immunoblotting analysis}

To extract proteins, the cultured cells were lysed using SDS lysis buffer (100 mM Tris- $\mathrm{HCl}, \mathrm{pH} 6.8,10 \%$ glycerol, and $1 \%$ SDS) supplemented with a protease inhibitor cocktail (Thermo Fisher Scientific, 78441). The protein concentration was determined using the BCA protein assay kit (Thermo Fisher Scientific, 23225). The samples were boiled in $1 \times$ sample buffer $(10 \mathrm{mM}$ Tris- $\mathrm{HCl}(\mathrm{pH} 6.8), 1 \%$ SDS, $5 \%$ glycerol, $0.05 \%$ bromophenol blue, and $1 \% \beta$-mercaptoethanol) for $5 \mathrm{~min}$ and subjected to SDSpolyacrylamide gel electrophoresis (SDS-PAGE). The resolved proteins were 
electrotransferred to an Immobilon-P membrane (Merck, IPVH00010). The membrane was probed with specific antibodies listed in Supplementary Table 3. Immunoreactive signals were detected using a LAS-4000 Luminescent Image Analyzer (GE Healthcare Bio-Sciences). The signal intensity was assessed by measuring the relative density of each band and normalizing it to that of Actb using the Multi Gauge software (Fujifilm).

\section{Statistical analysis}

All data, except RNA sequencing data, were analyzed using GraphPad Prism v5.02 software (GraphPad, La Jolla, CA). The data are represented as mean \pm standard error unless specified otherwise. The means were compared using the Student's t-test or two-way analysis of variance (ANOVA), followed by $\mathrm{h}$ Bonferroni's post-hoc test as indicated in each figure legend. The changes in the histological scores before and after treatment were analyzed using the Fisher's exact test and were compared with those of the vehicle group. All other parameters were analyzed using one-way ANOVA, followed by Dunnett's post-hoc test. Differences were considered significant at $P<0.05$ 


\section{REFERENCES}

1. Barb D, Portillo-Sancchez P \& Cusi K. Pahrmacological management of nonalcoholic fatty liver disease. Metabolism 65, 1183-1195 (2016).

2. Chen $Z$, et al. A vicious circle between insulin resistance and inflammation in nonalcoholic fatty liver disease. Lipids Health Dis 16, 203 (2017).

3. Baker P \& Friedman J. Mitochondrial role in the neonatal predisposition to developing nonalcoholic fatty liver disease. J Clin Invest 128, 3692-3703 (2018).

4. Barr J, et al. Obesity-dependent metabolic signatures associated with nonalcoholic fatty liver disease progression. J Proteome Res 11, 2521-2532 (2012).

5. Haas J, Francque $S \&$ Staels B. Pathophysiology and mechanisms of nonalcoholic fatty liver disease. Annu Rev Physiol 78, 181-205 (2016).

6. Vassilatou E. Nonalcoholic fatty liver disease and polycystic ovary syndrome. World J Gastroenterol 20, 8351-8363 (2014).

7. Armstrong $\mathrm{M}$, et al. Liraglutide safety and efficacy in patients with non-alcoholic steatohepatitis (LEAN): a multicentre, double-blind, randomized, placebocontrolled phase 2 study. Lancet 387, 679-690 (2016).

8. Drucker D. Mechanisms of action and therapeutic application of glucagon-like peptide-1. Cell Metab 27, 740-756 (2018).

9. Flock $\mathrm{G}$, et al. Incretin receptors for glucagon-like peptide 1 and glucosedependent insulinotropic polypeptide are essential for the sustained metabolic actions of vildagliptin in mice. Diabetes 56, 3006-3013 (2007).

10. Ao M, et al. Factors influencing glabridin stability. Nat Prod Coomun 5, 1907- 
1912 (2010).

11. Belinky $P$, et al. The antioxidative effects of the isoflavan glabridin on endogenous constituents of LDL during its oxidation. Atherosclerosis 137, 4961 (1998).

12. Yehuda I, et al. Glabridin, an isoflavan from licorice root, downregulates iNOS expression and activity under high-glucose stress and inflammation. Mol Nutr Food Res 59, 1041-1052 (2015).

13. Newsome PN, et al. A Placebo-Controlled Trial of Subcutaneous Semaglutide in Nonalcoholic Steatohepatitis. N Engl J Med 384, 1113-1124 (2021).

14. Singh R, et al. Autophagy regulates lipid metabolism. Nature 458, 1131-1135 (2009).

15. Chen Z, et al. Role of oxidative stress in the pathogenesis of nonalcoholic fatty liver disease. Free Radic Biol Med 152, 116-141 (2020).

16. Schofield JH \& Schafer ZT. Mitochondrial reactive oxygen species and mitophagy: a complex and nuanced relationship. Antioxid Rdox Signal 34, 517530 (2021).

17. Sun $\mathrm{N}$, et al. A fluorescence-based imaging method to measure in vitro and in vivo mitophagy using mt-Keima. Nat Protoc 12, 1576-1587 (2017).

18. Horke $\mathrm{S}$, et al. Paraoxonase-2 reduces oxidative stress in vascular cells and decreases endoplasmic reticulum stress-induced caspase activation. Circulation 115, 2055-2064 (2007).

19. Devarajan A, et al. Paraoxonase 2 deficiency alters mitochondrial function and exacerbates the development of atherosclerosis. Antiox Redox Signal 14, 341$351(2011)$ 
20. $\mathrm{Ng} \mathrm{CJ}$, et al. Adenovirus mediated expression of human Paraoxonase 2 protects against the development of atherosclerosis in apolipoprotein Edeficient mice. Mol Genet Metab 89, 368-373 (2006).

21. Witte I, et al. Protectors or Traitors: The role of PON2 and PON3 in atherosclerosis and cancer. J Lipids 2012, 1-12 (2012).

22. Ahn J, et al. Anti-obesity effects of glabridin-rich supercritical carbon dioxide extract of licorice in high-fat-fed obese mice. Food Chem Toxicol 51, 439-445 (2013).

23. Wu F, Jin Z \& Jin J. Hypoglycemic effects of glabridin, a polyphenolic flavonoid from licorice, in an animal model of diabetes mellitus. Mol Med Rep 7, 1278$1282(2013)$.

24. Boland M, et al. Towards a standard diet-induced and biopsy-confirmed mouse model of non-alcoholic steatohepatitis: Impact of dietary fat source. World $\mathrm{J}$ Gastroenterol 25, 4904-4920 (2019).

25. Roth J, et al. Combined obeticholic acid and elafibranor treatment promotes additive liver histological improvements in a diet-induced ob/ob mouse model of biopsy-confirmed NASH. Sci Rep 9, 9046 (2019).

26. Gorden D, et al. Biomarkers of NAFLD progression: a lipidomics approach to an epidemic. J Lipid Res 56, 722-736 (2015).

27. Puri $P$, et al. A lipidomic analysis of nonalcoholic fatty liver disease. Hepatology 46, $1081-1090$ (2007).

28. Ma D, et al. Plasma phospholipids and fatty acid composition differ between liver biopsy-proven nonalcoholic fatty liver disease and healthy subjects. Nutr Diabetes 6, e220 (2016). 
29. Tiwari-Heckler S, et al. Circulating phospholipid patterns in NAFLD patients associated with a combination of metabolic risk factors. Nutrients 10, 649 (2018).

30. Hollie $\mathrm{N}$, et al. Micromolar changes in lysophosphatidylcholine concentration cause minor effects on mitochondrial permeability but major alterations in function. Biochim Biophys Acta 1841, 888-895 (2014).

31. Kakisaka K, et al. Mechanisms of lysophosphatidylcholine-induced hepatocyte lipoapoptosis. Am J Physiol Gastrointest Liver Physiol 302, G77-84 (2012).

32. Hirsova $P$, et al. Lipid-induced signaling causes release of inflammatory extracellular vesicles from hepatocytes. Gastroenterology 150, 959-967 (2016).

33. Allard $\mathrm{J}$, et al. Nutritional assessment and hepatic fatty acid composition in non-alcoholic fatty liver disease (NAFLD): a cross-sectional study. J Hepatol 48, 300-307 (2008).

34. Ma D, et al. Plasma phospholipids and fatty acid composition differ between liver biopsy-proven nonalcoholic fatty liver disease and healthy subjects. Nutr Diabetes 18, e220 (2016).

35. Puri $P$, et al. A lipidomic analysis of nonalcoholic fatty liver disease. Hepatology 46, 1081-1090 (2007).

36. López-Vicario $C$, et al. Molecular interplay between $\Delta 5 / \Delta 6$ desaturases and long-chain fatty acids in the pathogenesis of non-alcoholic steatohepatitis. Gut 63, 344-355 (2014).

37. Zeng $X$, et al. Oleic acid ameliorates palmitic acid induced hepatocellular lipotoxicity by inhibition of ER stress and pyroptosis. Nutr Metab (Lond) 17, 11 (2020). 
38. Yan S, et al. Relevance of autophagy to fatty liver diseases and potential therapeutic applications. Amino Acids 49, 1965-1979 (2017).

39. Perrotta I \& Aquila S. The role of oxidative stress and autophagy in atherosclerosis. Oxid Med Cell Longev 2015, 130315 (2015).

40. Zhang Q, et al. ER stress and autophagy dysfunction contribute to fatty liver in diabetic mice. Int J Biol Sci 11, 559-568 (2015).

41. Martinez-Useros J, et al. Obesity and colorectal cancer: molecular features of adipose tissue. J Transl Med 14, 21 (2016).

42. Schott M, et al. Lipid droplet size directs lipolysis and lipophagy catabolism in hepatocytes. J Cell Biol 218, 3320-3335 (2019).

43. Wang $\mathrm{Y}$, et al. Macroautophagy and chaperone-mediated autophagy are required for hepatocyte resistance to oxidant stress. Hepatology 52, 266-277 (2010).

44. Amir M, et al. Inhibition of hepatocyte autophagy increases tumor necrosis factor-dependent liver injury by promoting caspase-8 activation. Cell Death Differ 20, 878-887 (2013).

45. Porcu C, et al. Oleuropein induces AMPK-dependent autophagy in NAFLD mice, regardless of the gender. Int J Mol Sci 19, 3948 (2018).

46. Sidarala $V$, et al. Mitophagy protects $\beta$ cells from inflammatory damage in diabetes. JCI Insight 5, e141138 (2020).

47. Zhang J. Autophagy and mitophagy in cellular damage control. Redox Biol 1, 19-23 (2013).

48. Kang R, et al. A novel PINK1- and PARK2-dependent protective neuroimmune pathway in lethal sepsis. Autophagy 12, 2374-2385 (2016). 
49. Yang S, et al. Defective mitophagy driven by dysregulation of rheb and KIF5B contributes to mitochondrial-reactive oxygen species (ROS)-induced nod-like receptor 3 (NLRP3) dependent proinflammatory response and aggravates lipotoxicity. Redox Biol 3, 63-71 (2014).

50. Villa E, Marchetti S \& Ricci J. No parkin zone: mitophagy without parkin. Trends Cell Biol 28, 882-895 (2018).

51. Garcia D, et al. Genetic liver-specific AMPK activation protects against dietinduced obesity and NAFLD. Cell Rep 26, 192-208 (2019).

52. Hussein $\mathrm{O}$, et al. Paraoxonase activity and expression is modulated by therapeutics in experimental rat nonalcoholic fatty liver disease. Int $\mathrm{J}$ Hepatol 2012, 265305 (2012).

53. Shih D, et al. PON2 deficiency leads to increased susceptibility to diet-induced obesity. Antioxidants 8, 19 (2019).

54. Camps J, Marsillach J \& Joven J. The paraoxonases: role in human diseases and methodological difficulties in measurement. Crit Rev Clin Lab Sci 46, 83106 (2009).

55. Mochizuki $\mathrm{H}$, et al. Human PON2 gene at 7q21.3: cloning, multiple mRNA forms, and missense polymorphisms in the coding sequence. Gene 13, 149157 (1998).

56. Draganov D, et al. Human paraoxonases (PON1, PON2, and PON3) are lactonases with overlapping and distinct substrate specificities. J Lipid Res 46, 1239-1247 (2005).

57. $\mathrm{Ng} \mathrm{C}$, et al. Paraoxonase- 2 is a ubiquitously expressed protein with antioxidant properties and is capable of preventing cell-mediated oxidative modification of 
low density lipoprotein. J Biol Chem 276, 44444-44449 (2001).

58. Manco G, Porzio E \& Carusone T. Human Paraoxonase-2 (PON2): protein functions and modulation. Antioxidants (Basel) 10, 256 (2021).

59. Bingol B, et al. The mitochondrial deubiquitinase USP30 opposes parkinmediated mitophagy. Nature 510, 370-375 (2014). 
Acknowledgments: This study was supported by the National Research Foundation of Korea (NRF) grants funded by the Korean government (NRF-2020R1A2C3010511, NRF-2021M3A9I2080488, and NRF-2021M3A9H3017086).

Author Contributions: G.C.S., K.P.K., and K.H.K. designed the experiments and analyzed the data; G.C.S., H.M.L., S.K.Y., D.R., Y.S.P., H.S.P., and N.Y.K. performed the experiments and analyzed the bioinformatics data. All authors discussed the results and contributed to the data analysis; G.C.S. and K.H.K. prepared the manuscript with contributions from all authors.

Competing interests: HML, SY, YSP, and HSP are current employees of Glaceum Inc. and hold its stocks/shares. Glaceum Inc. holds the intellectual property rights of the SGDs. The remaining authors declare no competing interests.

Data and materials availability: All data supporting the findings of this study are available within the article and its Supplementary Information files and from the corresponding author upon reasonable request. A reporting summary for this article is available as a Supplementary Information file. 


\section{Figure Legends}

Fig. 1 Synthetic glabridin derivatives (SGDs) mitigate steatosis and inflammation in a biopsy-confirmed rodent non-alcoholic steatosis hepatitis (NASH) model. a Mice were fed with amylin (AMLN) diet and NASH was confirmed using biopsy. Next, the mice were orally administered with HSG4112 (100 mg/kg bodyweight) and HSG4113 (200 $\mathrm{mg} / \mathrm{kg}$ bodyweight) for 6 weeks once daily. Representative results of hematoxylin and eosin, oil red $\mathrm{O}$, Sirius red staining and Lgals3, Acta, Adgre1 immunostaining (scale bar $=50 \mu \mathrm{m})$. The histopathological scores are shown above. b Quantification of plasma total cholesterol, liver cholesterol, and serum Tnfa, alanine aminotransferase, aspartate aminotransferase, and alkaline phosphatase. c-f Histological scores of nonalcoholic fatty liver disease activity (c), lobular inflammation (d), steatosis (e), and ballooning degeneration (f) in biopsies before treatment (pre) and after treatment (post) are shown in the left. Numbers of animals exhibiting exacerbation (higher), no change (same), and alleviation (lower) of disease after treatment relative to before treatment are shown in the right. g-i Effect of SGDs on bodyweight (g), liver weight (h), and cumulative food intake (i). Data are presented as mean \pm standard deviation ( $n=12$ for the vehicle-treated group; $n=11$ for the HSG4112-treated group; $n=10$ for the HSG4113-treated group). In $\mathbf{a}, \mathbf{b}$, and $\mathbf{h}$, data of the SGD-treated and vehicle-treated groups were compared using one-way analysis of variance, following by Dunnett's post-hoc test. In c-f, data were analyzed using the Fisher's test, followed by the Bonferroni test.

Fig. 2 Therapeutic effect of HSG4112 on non-alcoholic steatosis hepatitis (NASH) is similar to that of semaglutide. a Amylin (AMLN) diet-fed mice were treated once daily 
with HSG4112 (50 or 100 mg/kg bodyweight, oral administration) and semaglutide (30 $\mathrm{nM} / \mathrm{kg}$ bodyweight, subcutaneous injection) for 10 weeks. Representative results of hematoxylin, oil red $\mathrm{O}$, and Sirius red staining, Lgals3, Acta2, and Adgre1 immunostaining (scale bars $=50 \mu \mathrm{m}$ ), and histopathological scores are shown above. b Quantification of plasma total cholesterol, liver cholesterol, liver Ccl2, and serum alanine aminotransferase (ALT), aspartate aminotransferase (AST), and alkaline phosphatase (ALP). c and d Histological scores of NAFLD activity (c) and lobular inflammation (d) in biopsies before treatment (pre) and after treatment (post) are shown on the left. Numbers of animals exhibiting exacerbation (higher), no change (same), and alleviation (lower) of disease after treatment relative to before treatment are shown right. e-i Effect of HSG4112 and semaglutide on the liver weight (e), bodyweight (f), fat/non-fat mass (g), muscle weight (h), and cumulative food intake (i). Data are presented as mean \pm standard deviation ( $n=15$ for the vehicle-treated group; $n=15$ for the HSG4112 (50 mg/kg bodyweight)-treated group; $n=14$ for the HSG4112 (100 mg/kg bodyweight)-treated group; $n=14$ for the semaglutide-treated group). In a, b, e, g, h, and I, data of the HSG4112-treated group vs. vehicle-treated group and semaglutide-treated group vs. vehicle-treated were compared using one-way analysis of variance, followed by Dunnett's post-hoc test. In $\mathbf{c}$ and $\mathbf{d}$, data were compared using the Fisher's test, followed by the Bonferroni test.

Fig. 3 HSG4112 and semaglutide differentially affect the lipid profiles. a Principal coordinate analysis of hepatic levels of total lipids in the vehicle-treated, HSG4112treated, and semaglutide-treated amylin (AMLN) diet-fed mice. b Volcano plot analysis of the hepatic levels of total lipids in the HSG4112-treated (left panel) and semaglutide- 
treated (right panel) groups when compared with those in the vehicle-treated group. Venn diagram showing the number of differentially regulated hepatic lipids in the HSG4112-treated and semaglutide-treated group relative to the vehicle-treated group. c Analysis of hepatic lipid classes in the vehicle-treated, HSG4112-treated, and semaglutide-treated groups. In the liver of the vehicle-treated group (top panel), the schematic diagram shows the relative levels of incorporation of exogenous fatty acids into sphingolipids and glycerophospholipids. Lipid classes identified using liquid chromatography-tandem mass spectrometry analysis are presented as color-coded circles. The lipid classes were designated as saturated if all their fatty acid chains were saturated and as unsaturated if they had at least one unsaturated fatty acid chain. The percentage of saturated lipid species is shown for each class from green (low saturation) to red (high saturation). Lipid classes not identified are shown in gray. The size of the circles is set to the arbitrary unit of 1 for the control cells. The hepatic lipid species in the HSG4112-treated and semaglutide-treated groups are presented in the middle and bottom panels, respectively. The size of the circle is proportional to the fold change in the content of lipid species in the drug-treated group relative to the vehicletreated group. d Forest plots showing individual hepatic lipid species in the HSG4112treated and semaglutide-treated AMLN diet-fed mice expressed as a fold change relative to the vehicle-treated group. Plots in blue or red represent differential individual lipid species between the drug-treated and vehicle-treated groups; $p<0.05$. e Analysis of the levels of enzymes involved in hepatic lipid metabolism in the HSG4112-treated and semaglutide-treated AMLN diet-fed mice when compared with those in the vehicletreated group. f Heatmap analysis of the correlation between lipid classes and pathological features in the HSG4112-treated and semaglutide-treated AMLN diet-fed 
mice. Each cell is color-coded and the Pearson's correlation coefficient is shown. The legend for color-coding is shown below. LPAs, lyso-phosphatidic acids; PAs, phosphatidic acids; MAG, monoacylglycerol; DAG, diacylglycerol; TAG, triacylglycerol; PC, phosphatidylcholine; LPC, lyso-phosphatidylcholine; PE, phosphatidylethanolamine; LPE, lyso-phosphatidylethanolamine; PI, phosphatidylinositol; LPI, lyso-phosphatidylinositol; PG, phosphatidylglycerol; LPG, lyso-phosphatidylglycerol; Cer, ceramide; SM, sphingomyelin; CerP, ceramide-1phosphate; Sa, sphinganine; Sa1P, sphinganine-1-phosphate; So, sphingosine; So1P, sphingosine-1-phosphates. Fabp4, fatty acid-binding protein 4; Fabp5, fatty acidbinding protein 5; Soat1, sterol O-acyltransferase 1; Agpat5, 1-acylglycerol-3phosphate O-acyltransferase 5; Agpat1, 1-acylglycerol-3-phosphate O-acyltransferase 1; Cdipt, CDP-diacylglycerol-inositol 3-phosphatidyltransferase; Cds1, CDPdiacylglycerol synthase 1; Dgke, diacylglycerol kinase epsilon; Mogat1, monoacylglycerol O-acyltransferase 1; Lipc, lipase C hepatic type; Plcg1, phospholipase C gamma 1; Lcat, lecithin-cholesterol acyltransferase; Lpl, lipoprotein lipase; Pla2g4a, phospholipase A2 group IVA; Pla2g15, phospholipase A2 group XV; Pitpnb, phosphatidylinositol transfer protein beta; CerK1, ceramide kinase $1{ }^{*} p<0.05$. Error bars indicate standard deviation.

Fig. 4 Transcriptome and phosphoproteome analyses indicated that HSG4112 exerts therapeutic effects by promoting autophagy activation. a Volcano plot of hepatic differentially expressed genes (DEGs) between the HSG4112-treated/semaglutidetreated and vehicle-treated amylin (AMLN) diet-fed mice. Genes upregulated or downregulated by more than 2 -fold are shown in red and blue, respectively. b Venn 
diagram showing the number of hepatic DEGs between the HSG4112treated/semaglutide-treated and vehicle-treated groups. c Two-dimensional hierarchical clustering of DEGs between different pairs of HSG4112-treated, semaglutide-treated, and vehicle-treated groups. The figure shows the most significant Gene Ontology (GO) biological processes for each cluster of genes upregulated (left panel) and downregulated (right panel) in the HSG4112-treated and semaglutidetreated groups (individually or in both groups) relative to the vehicle-treated group. Red and green denote highly and weakly expressed genes, respectively. d Violin plots showing the mean and variance of overlapping gene clusters between drug-treated and vehicle-treated groups. e and f Among HSG4112-specific gene clusters, the relative abundance scores of upregulated (e) and downregulated (f) genes were compared between all pairs of HSG4112-treated, semaglutide-treated, and vehicle-treated groups. Bar plots showing the average fold change in expression of perturbed genes in HSG4112-specific gene clusters. g Top-ranked pathways in phosphoproteome analysis that are significantly altered in the HSG4112-treated and semaglutide-treated groups relative to the vehicle-treated group. Red cells represent the pathways enriched by proteins exhibiting upregulated phosphorylation (Phospho) and corresponding protein expression (Global) in the drug-treated group relative to the vehicle-treated group. Blue cells represent the pathways enriched by the proteins exhibiting downregulated phosphorylation and corresponding total protein expression in the drug-treated groups relative to the vehicle-treated group. Each cell is color-coded based on the fold change in the expression of genes in the drug-treated groups relative to the vehicle-treated group. The legend for the color codes is shown below. Bold words represent major signaling pathways enriched by HSG4112-specific phosphoproteins. h Network 
scheme showing the interactions of genes involved in the autophagy signaling pathway. The genes/proteins are selected from the DEGs of the transcriptome and phosphoproteome analysis with enhanced connectivity and significant differential expression in the HSG4112-treated group. Each gene/protein symbol is color-coded based on the fold change in expression of genes/proteins in the drug-treated groups relative to the vehicle-treated groups. The legend for color-coding is shown below and represented with the phosphorylation site. ${ }^{*} p<0.05$. Error bars indicate standard deviation. N/A, not available.

Fig. 5 HSG4112 activates autophagy/mitophagy and alleviates lipotoxic oxidative stress. a L02 hepatocytes were treated with free fatty acids (FFAs) $(500 \mu \mathrm{M})$ alone or in combination with HSG4112 $(3 \mu \mathrm{M}) 72 \mathrm{~h}$ and stained with oil red $\mathrm{O}$ to determine lipid accumulation. Representative images from six independent experiments are shown above (scale bar $=50 \mu \mathrm{m}$ ). Quantification of oil red $\mathrm{O}$ staining intensities is shown below. b Immunoblotting analysis of autophagy flux activation-related markers (Map1lc3b, Sqstm1, and Actb) in L02 cells treated with FFAs alone or in combination with HSG4112 for indicated durations. Actb was used as a loading control. Band intensities of indicated proteins are shown below. c Confocal fluorescence analysis showing the puncta of tandem fluorescent probe-tagged LC3B (red fluorescent protein (mRFP)-green fluorescent protein (GFP)-LC3B). L02 cells were transfected with mRFP-GFP-LC3B plasmid and treated with FFAs alone or in combination with HSG4112 for $72 \mathrm{~h}$. Cells with both red and green fluorescent puncta (autophagosome) and those with only red fluorescent puncta (autolysosome) were quantified. Representative images from three independent experiments are shown above (scale 
bar $=20 \mu \mathrm{m})$. Bar plots of the average cell numbers with red fluorescent puncta (autophagy) or average numbers of autophagosome or autolysosome per cell are shown below. Error bars indicate standard deviation. d Confocal fluorescence analysis showing the puncta of mRFP-GFP-LC3B in cells treated with bafilomycin A (BFA), an autophagy maturation inhibitor. L02 cells transfected with mRFP-GFP-LC3B plasmid were treated with FFAs alone or in combination with HSG4112 for $72 \mathrm{~h}$, followed by treatment with BFA (100 nM) for $5 \mathrm{~h}$. Representative images from three independent experiments are shown above (scale bar $=20 \mu \mathrm{m}$ ). Bar plots of the average cell numbers exhibiting autophagy or average numbers of autophagosome or autolysosome per cell are shown below. Error bars indicate standard deviation. e Immunohistochemical staining analysis of hepatic Map1lc3b and Becn1 levels in HSG4112-treated amylin (AMLN) diet-fed mice. Representative images of hepatic sections of the vehicle-treated $(n=12)$ and HSG4112-treated $(n=11)$ mice are shown above (scale bar $=50 \mu \mathrm{m}$ ). Quantification of staining intensity (inverted mean pixel values) is shown below. $\mathrm{f}$ Confocal fluorescence images showing the generation of mitochondrial superoxide, which was analyzed using MitoSox staining. L02 cells were treated with palmitic acid (PA) $(125 \mu \mathrm{M})$ alone or in combination with HSG4112 $(3 \mu \mathrm{M})$ for $24 \mathrm{~h}$ and stained with MitoSox. Cell nuclei were stained with Hoechst 33342. Representative images from three independent experiments are shown in the left (scale bar $=20 \mu \mathrm{m}$ ). Bar plots of the quantification of MitoSox staining intensities are shown right. Error bars indicate standard deviation. $\mathbf{g}$ Quantification of lipid peroxidation in the liver and hepatocytes. Hepatic lipid peroxidation in the HSG4112treated $(n=13)$, semaglutide-treated $(n=12)$, and vehicle-treated $(n=15)$ AMLN dietfed mice was measured using the malondialdehyde (MDA) accumulation assay (top 
panel). Bar plots of the average lipid peroxidation in hepatocytes determined using the MDA (middle panel) and 4-hydroxynonenal assays (bottom panel). Lipid peroxidation in L02 cells treated with PA alone or in combination with HSG4112 for $24 \mathrm{~h}$. Data were obtained from three independent experiments. Error bars indicate standard deviation. $\mathbf{h}$ Immunoblotting analysis of mitophagy activation-related markers (Prkn, Pink1, Bnip3, Bnip3I, Map1lc3b, Sqstm1, and Actb) in L02 cells treated with PA alone or in combination with HSG4112 for $5 \mathrm{~h}$. Actb was used as a loading control. Band intensities of indicated proteins are shown below. i Confocal fluorescence analysis of mitophagy activation-related markers in hepatocytes. L02 cells were transfected with mt-Keima, a pH-dependent fluorescent mitophagy probe, were treated with $\mathrm{PA}$ alone or in combination with HSG4112 for $24 \mathrm{~h}$ and the mt-Keima fluorescent intensity was quantified. Green fluorescent filaments $(\mathrm{pH} 7.0)$ of mt-Keima indicate mitochondrial networks, while enlarged red fluorescence of mt-Keima $(\mathrm{pH} 4.0)$ indicates mitophagy flux (fused with lysosome). Representative images from three independent experiments are shown in the left (scale bar $=20 \mu \mathrm{m})$. Bar plots of the average red (excitation 561 $\mathrm{nm}$ ) and green (excitation $458 \mathrm{~nm}$ ) fluorescence intensities are shown on the right. Error bars indicate standard deviation. NS, non-significant.

Fig. 6 HSG4112 activates mitophagy and autophagy, reduces lipid accumulation, and maintains redox homeostasis through Pon2. a Confocal fluorescence images showing the mitochondrial localization of HSG4112. L02 cells were treated with Alexa488-azideconjugated propargyl-HSG4112 (3 $\mu \mathrm{M})$, which was prepared using the Click-iT reaction buffer kit, for $5 \mathrm{~h}$. Mitochondria were stained with MitoTracker Red. The experimental procedure is depicted above. Representative images from three independent 
experiments are shown below (scale bar $=20 \mu \mathrm{m}$ ). $\mathbf{b}$ Identification of target proteins of HSG4112 using chemical proteome analysis. Proteins extracted from L02 cells interacted with the propargyl-HSG4112-biotin-streptavidin bead complex. The experimental procedure is depicted on the left. Immunoblotting analysis of the interaction between HSG4112 and Pon2 using three independent samples is shown on the right. c Competitive binding assay to determine the specific interaction between HSG4112 and Pon2. Cell lysates or purified recombinant Pon2 protein were incubated with different concentrations of free HSG4112, followed by incubation with streptavidin bead-conjugated HSG4112. The experimental procedure is depicted on the left. Immunoblotting analysis of the specific interaction between HSG4112 and Pon2 is shown on the right. d Results of the enzymatic assay demonstrating the esterase and lactonase activities of Pon2 in hepatocytes. Pon2 activity in L02 cells treated with palmitic acid (PA) alone or in combination with HSG4112 for $24 \mathrm{~h}$. Bar plots of the average esterase and lactonase activities are shown on the left. Data were obtained from three independent experiments. Error bars indicate standard deviation. Immunoblot analysis of Pon2 and Actb is shown on the right. Actb was used as a loading control. Band intensities of the indicated proteins are shown below. e Pon2 activities in the Pon2 knockdown (KD) and control L02 cells treated with PA alone or in combination with HSG4112 for $24 \mathrm{~h}$. Bar plots of the average esterase and lactonase activities are shown on the left. Data were obtained from three independent experiments. Error bars indicate standard deviation. Immunoblotting analysis of Pon2 and Actb is shown on the right. Actb was used as a loading control. NS, non-significant. f Purified recombinant Pon2 activities incubated with oxidized linoleic acid (OX-LA) alone or in combination with HSG4112 for $10 \mathrm{~min}$. Bar plots of the average esterase 
and lactonase activities of recombinant Pon2. Data were obtained from three independent experiments. Error bars indicate standard deviation. g Confocal fluorescence images showing the generation of mitochondrial superoxide, which was analyzed using MitoSox. Pon2 KD and control L02 cells were treated with PA alone or in combination with HSG4112 for $24 \mathrm{~h}$ and stained with MitoSox. Cell nuclei were stained with Hoechst 33342. Representative images from three independent experiments are shown above (scale bar $=20 \mu \mathrm{m}$ ). Bar plots of the MitoSox staining intensity are shown below. Error bars indicate standard deviation. $\mathbf{h}$ The quantification of lipid peroxidation in Pon2 KD and control L02 cells treated with PA alone or in combination with HSG4112 for $24 \mathrm{~h}$. Bar plots of the average lipid peroxidation determined using the malonaldehyde accumulation (left panel) and 4-hydroxynonenal assays (right panel). Data were obtained from three independent experiments. Error bars indicate standard deviation. NS, non-significant. i Immunoblotting analysis of autophagy flux activation-related markers (Map1lc3b, Sqstm1, and Actb) in Pon2 KD and control L02 cells treated with free fatty acids (FFAs) alone or in combination with HSG4112 for $48 \mathrm{~h}$. Actb was used as a loading control. The band intensities of indicated proteins are shown below. $\mathbf{j}$ Immunoblotting analysis of mitophagy activationrelated markers (Prkn, Pink1, Bnip3, Bnip3I, and Map1lc3b) in Pon2 KD and control L02 cells treated with PA alone or in combination with HSG4112 for $24 \mathrm{~h}$. Actb was used as a loading control. Band intensities of indicated proteins are shown below. $\mathbf{k}$ Pon2 KD and control L02 cells were treated with FFAs alone or in combination with HSG4112 combined for $72 \mathrm{~h}$ and stained with oil red $\mathrm{O}$ to examine lipid accumulation. Representative images from five independent experiments are shown above (scale bar $=50 \mu \mathrm{m})$. Quantification of oil red O staining intensity is shown below. NS, non- 
bioRxiv preprint doi: https://doi.org/10.1101/2021.10.01.462722; this version posted October 1, 2021. The copyright holder for this preprint (which was not certified by peer review) is the author/funder, who has granted bioRxiv a license to display the preprint in perpetuity. It is made available under aCC-BY-ND 4.0 International license.

\section{significant.}



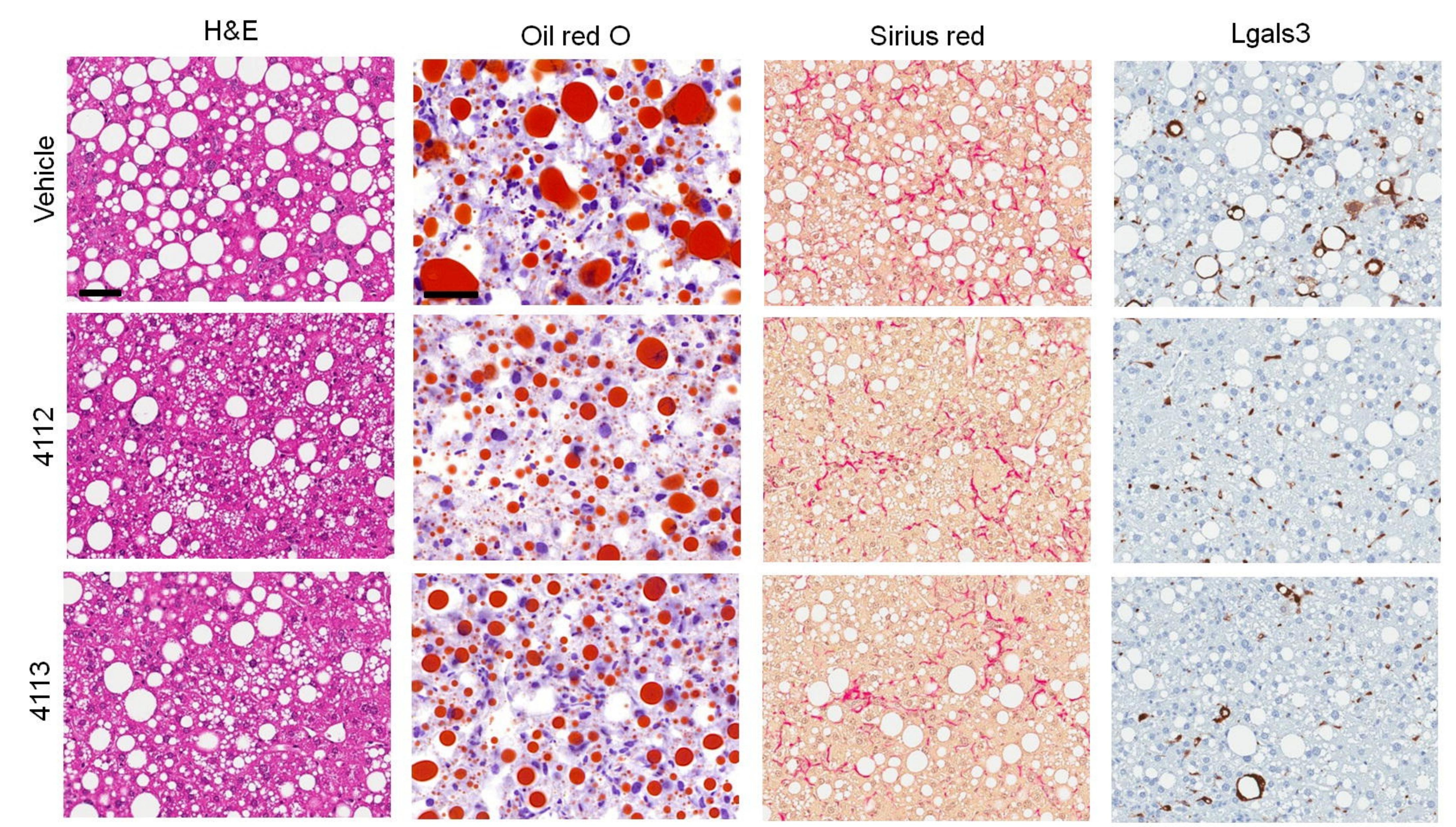

Acta2

Adgre1
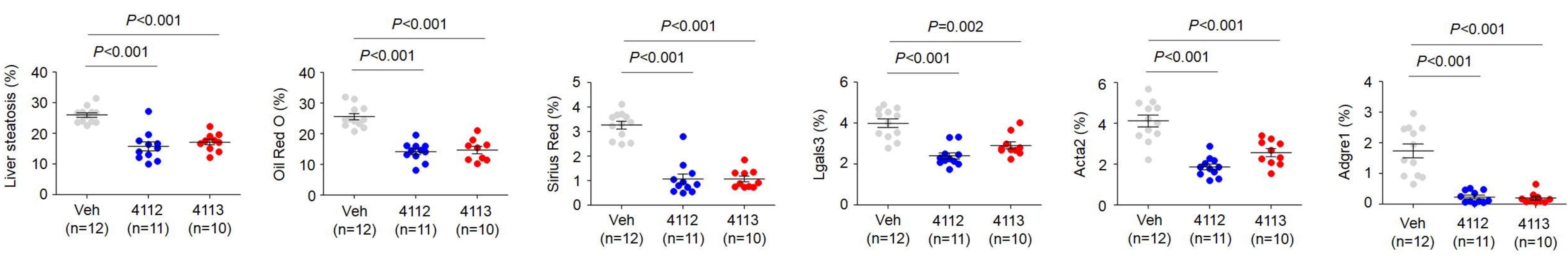

b
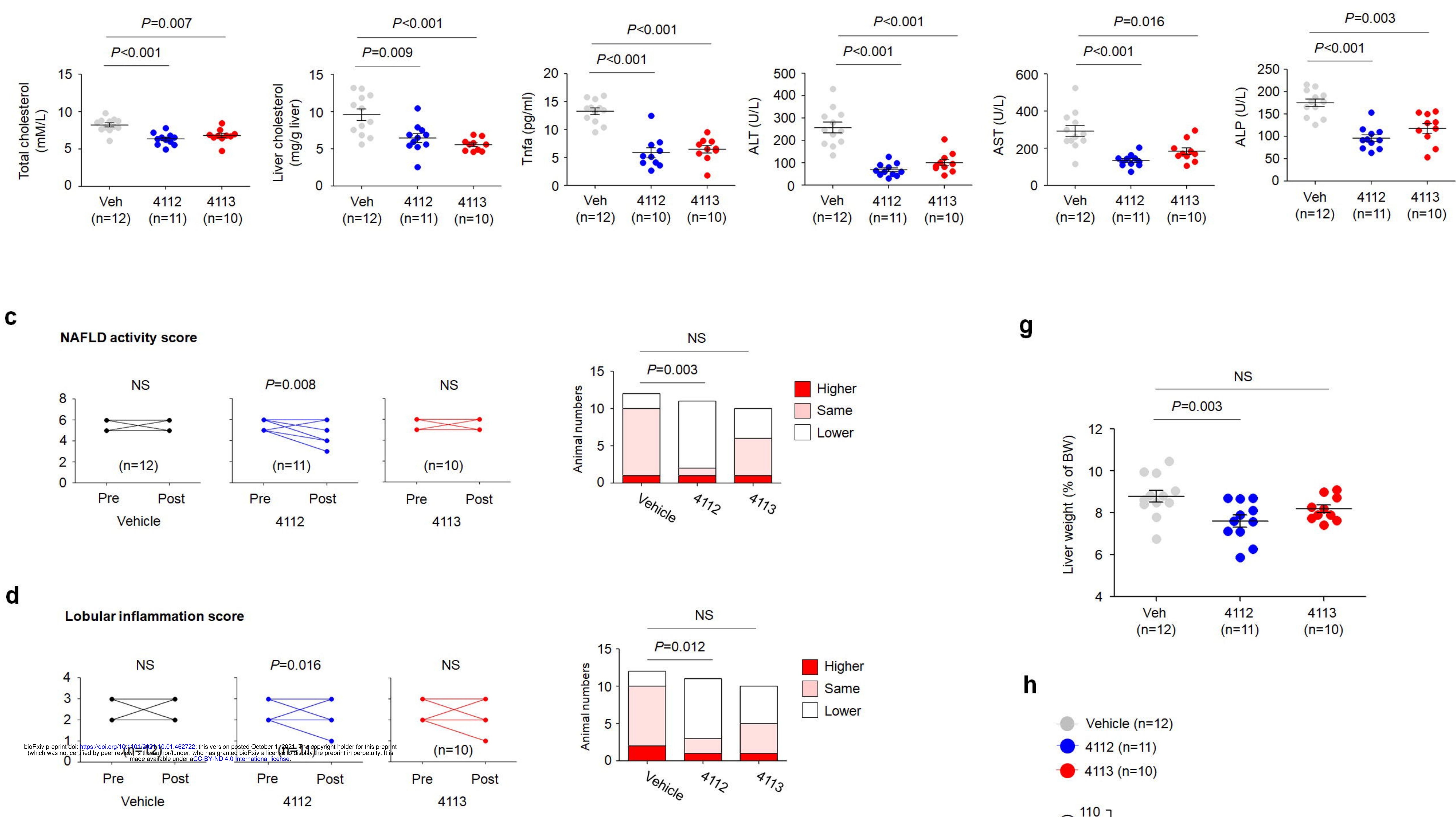

\section{g}

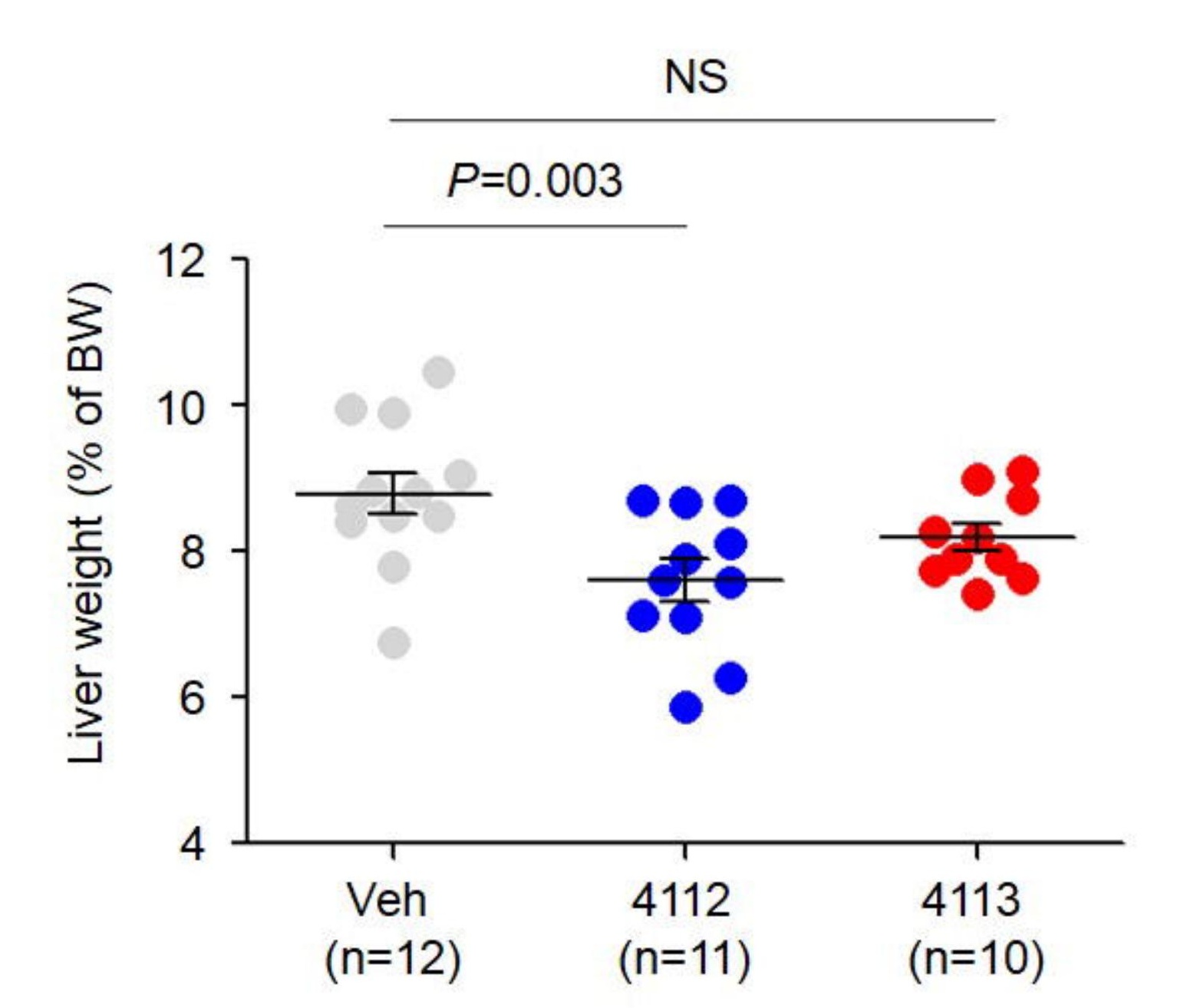

h

Vehicle $(\mathrm{n}=12$
$4112(\mathrm{n}=11)$
$4113(\mathrm{n}=10)$
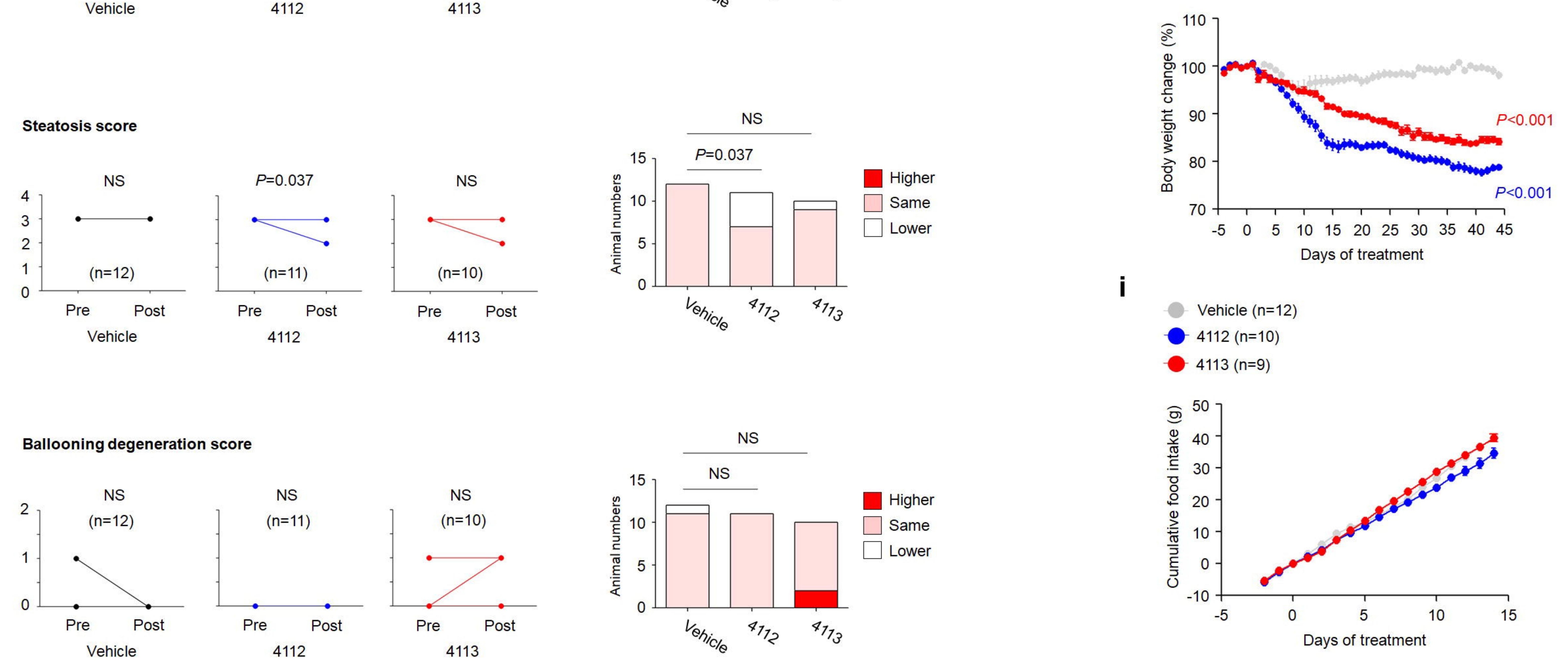

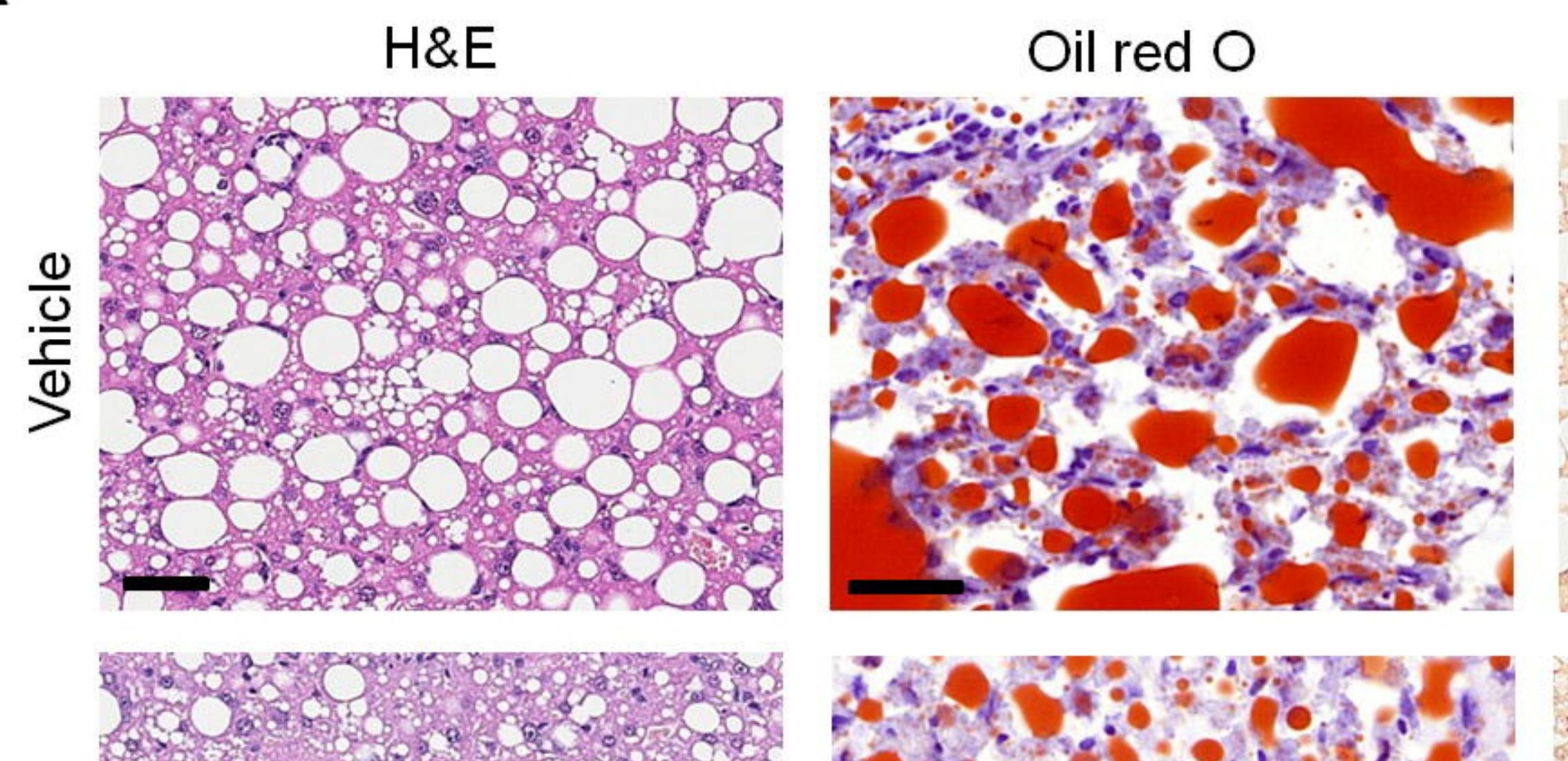

$\stackrel{5}{+}$

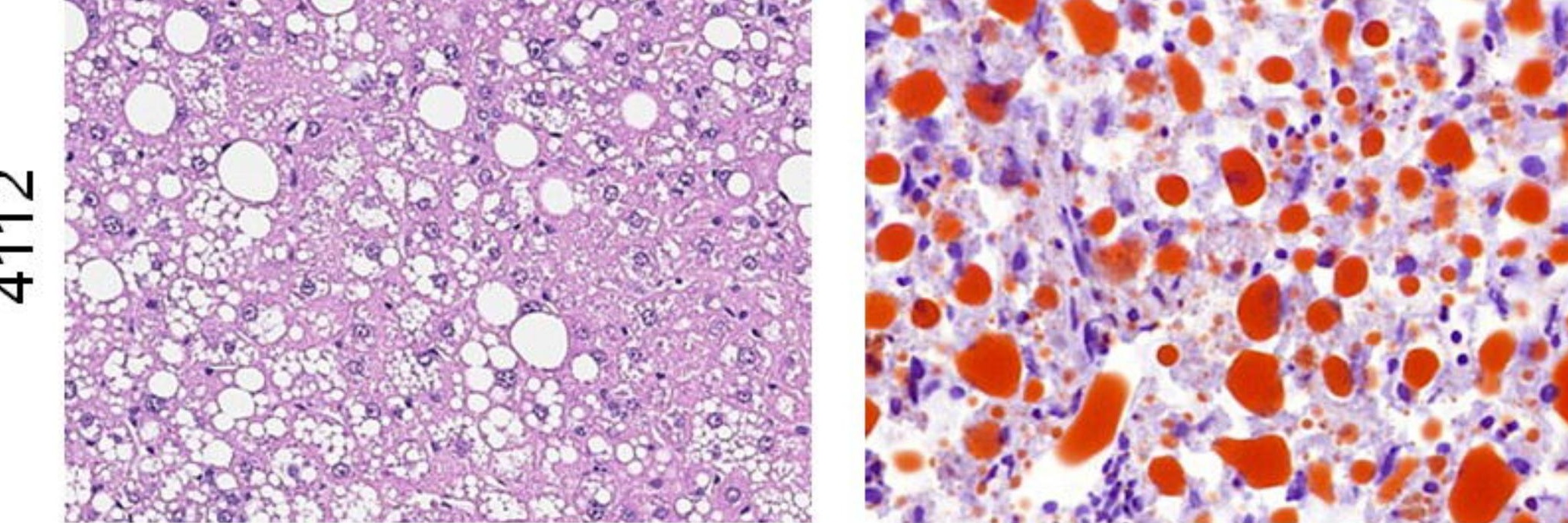

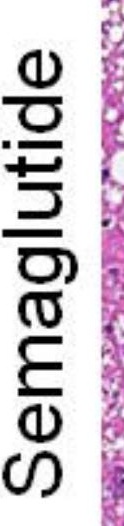
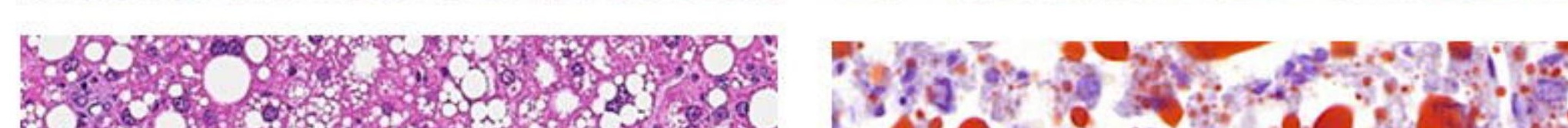
.
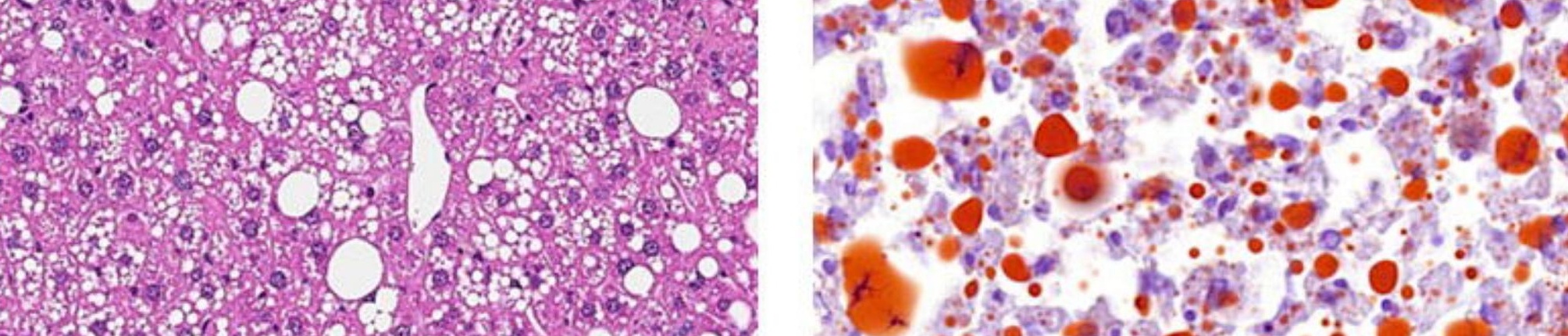

Mo. on

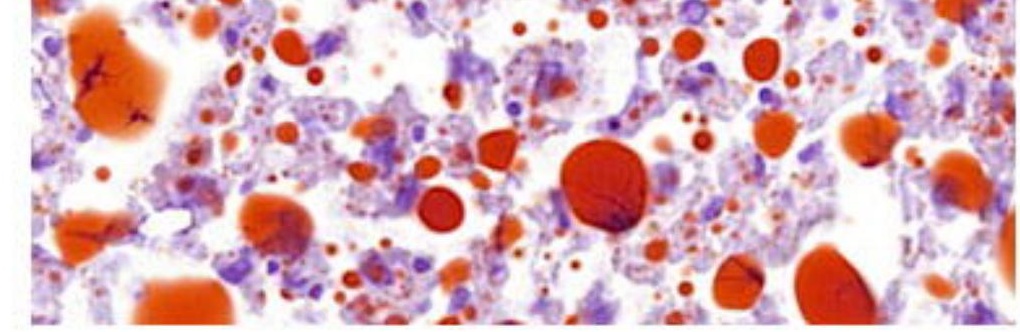

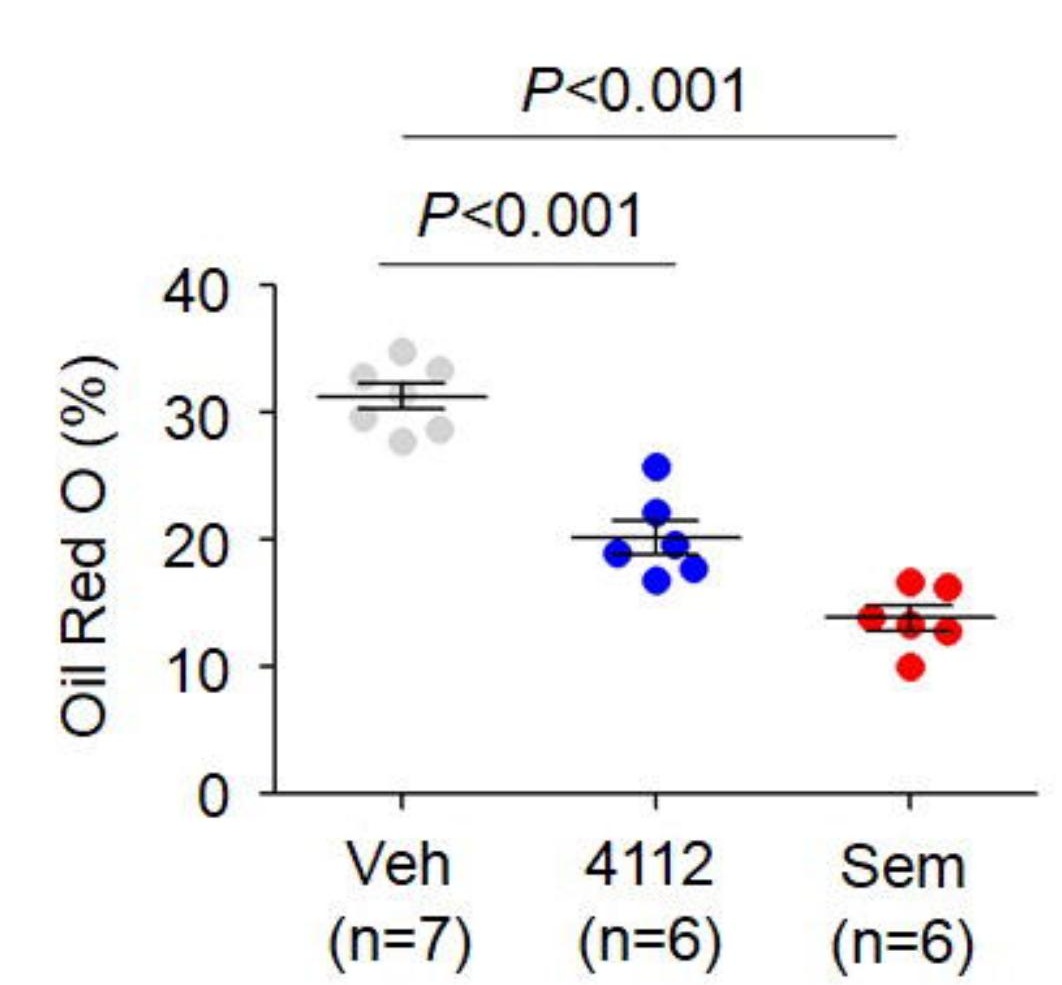

$\begin{array}{lll}(\mathrm{n}=7) & (\mathrm{n}=6) & \text { Sem } \\ (\mathrm{n}=6)\end{array}$

Sirius red

Lgals3
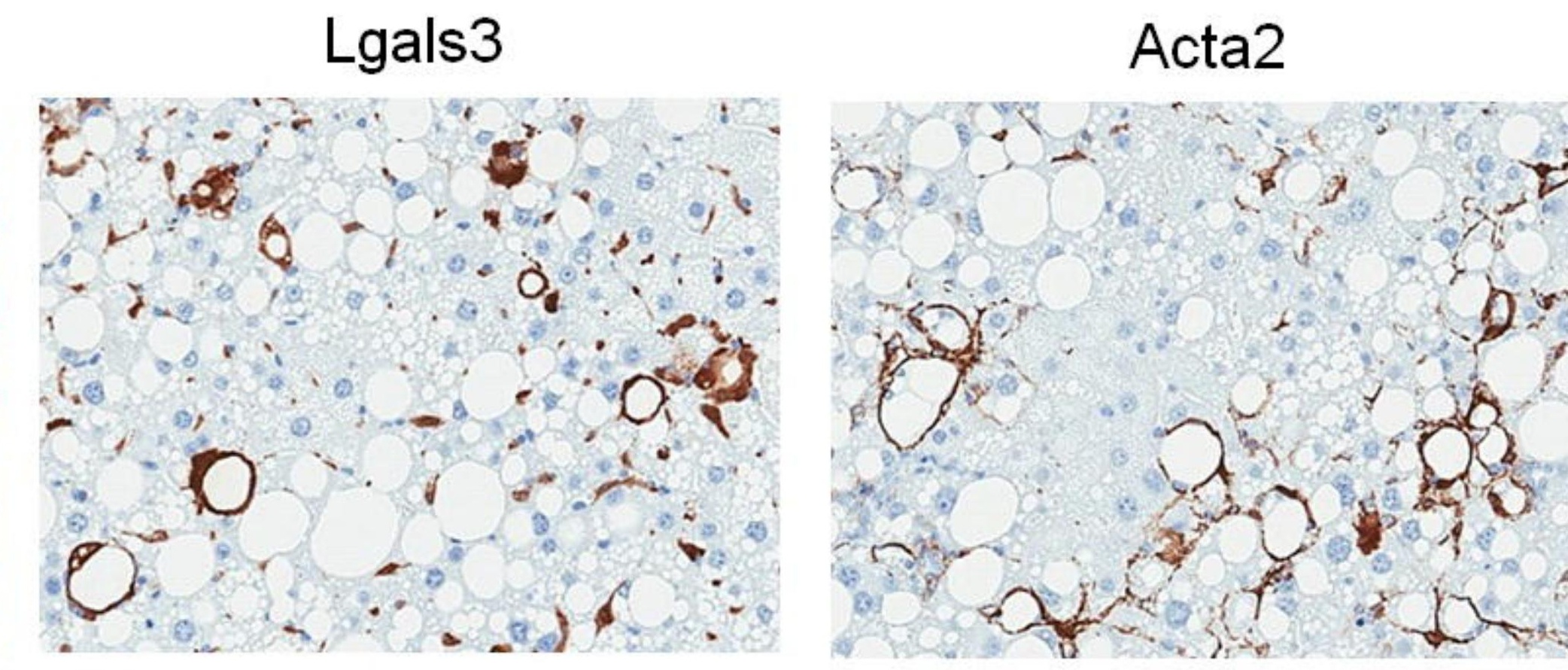

Adgre1
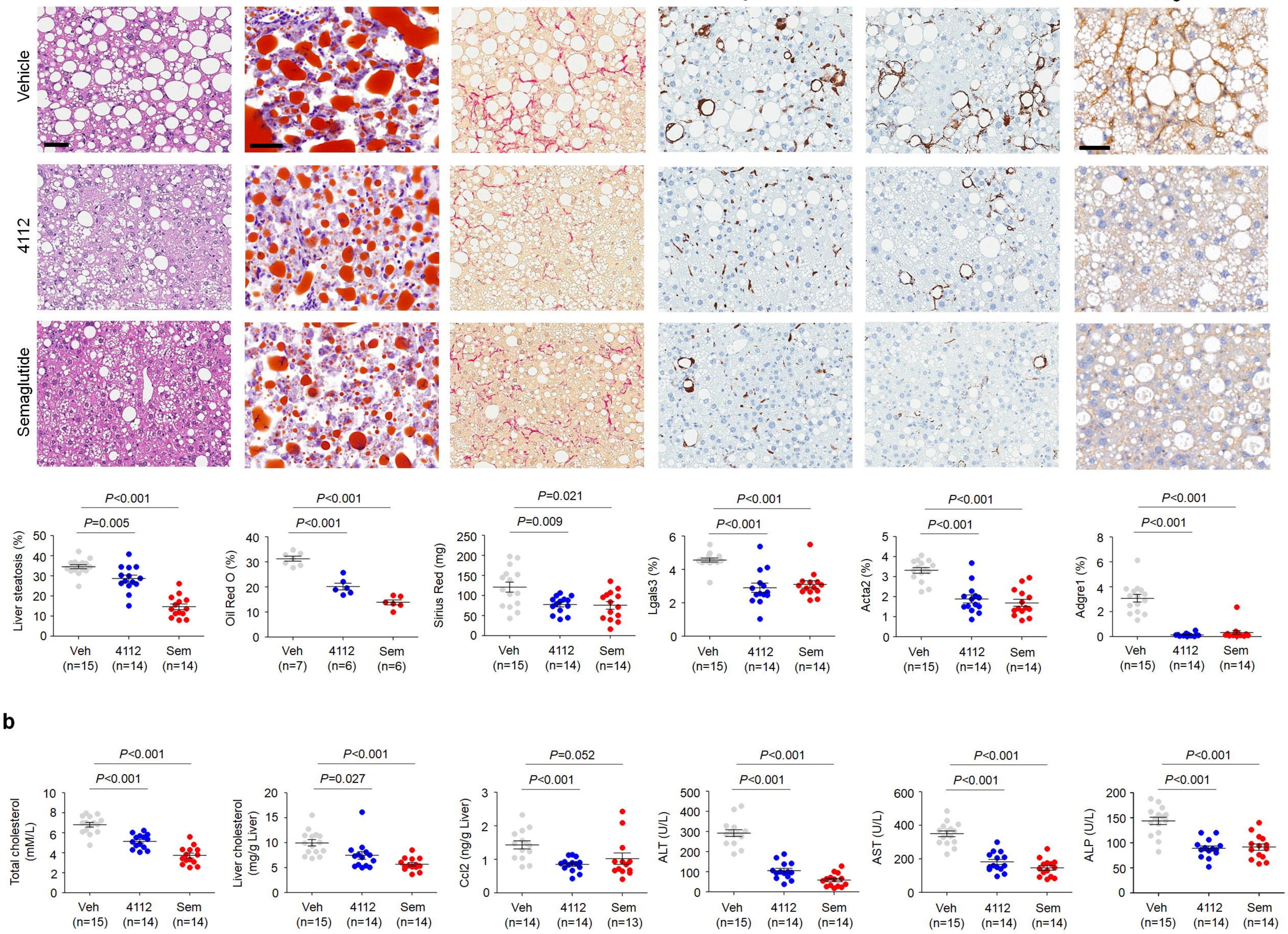

NAFLD activity score

8
6
4
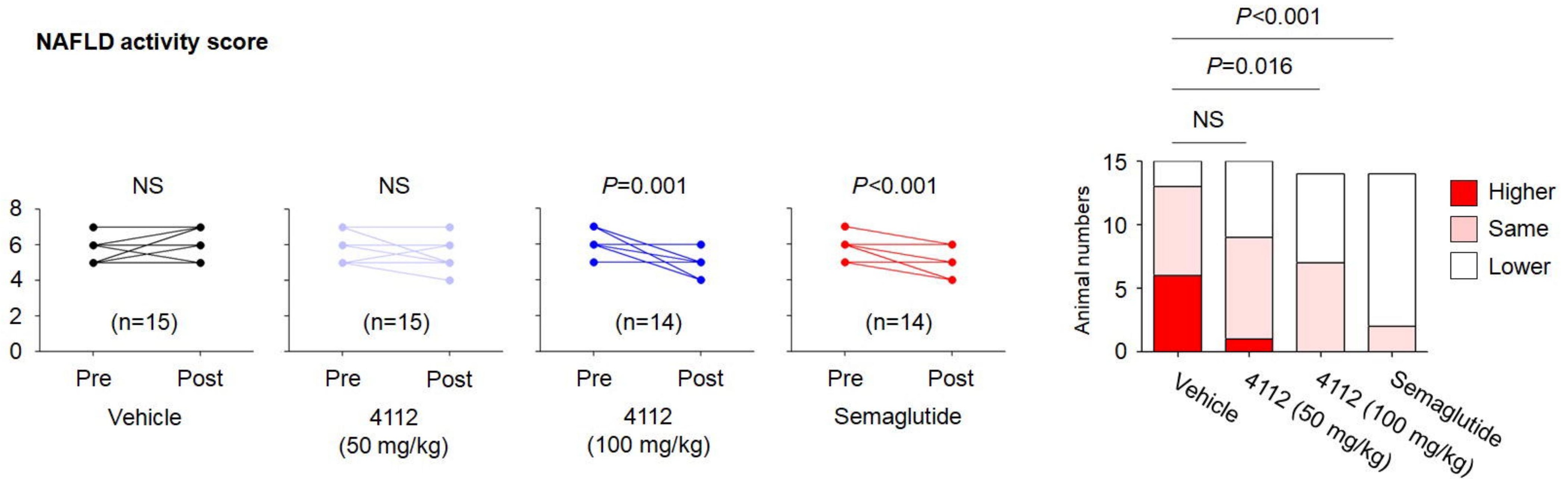

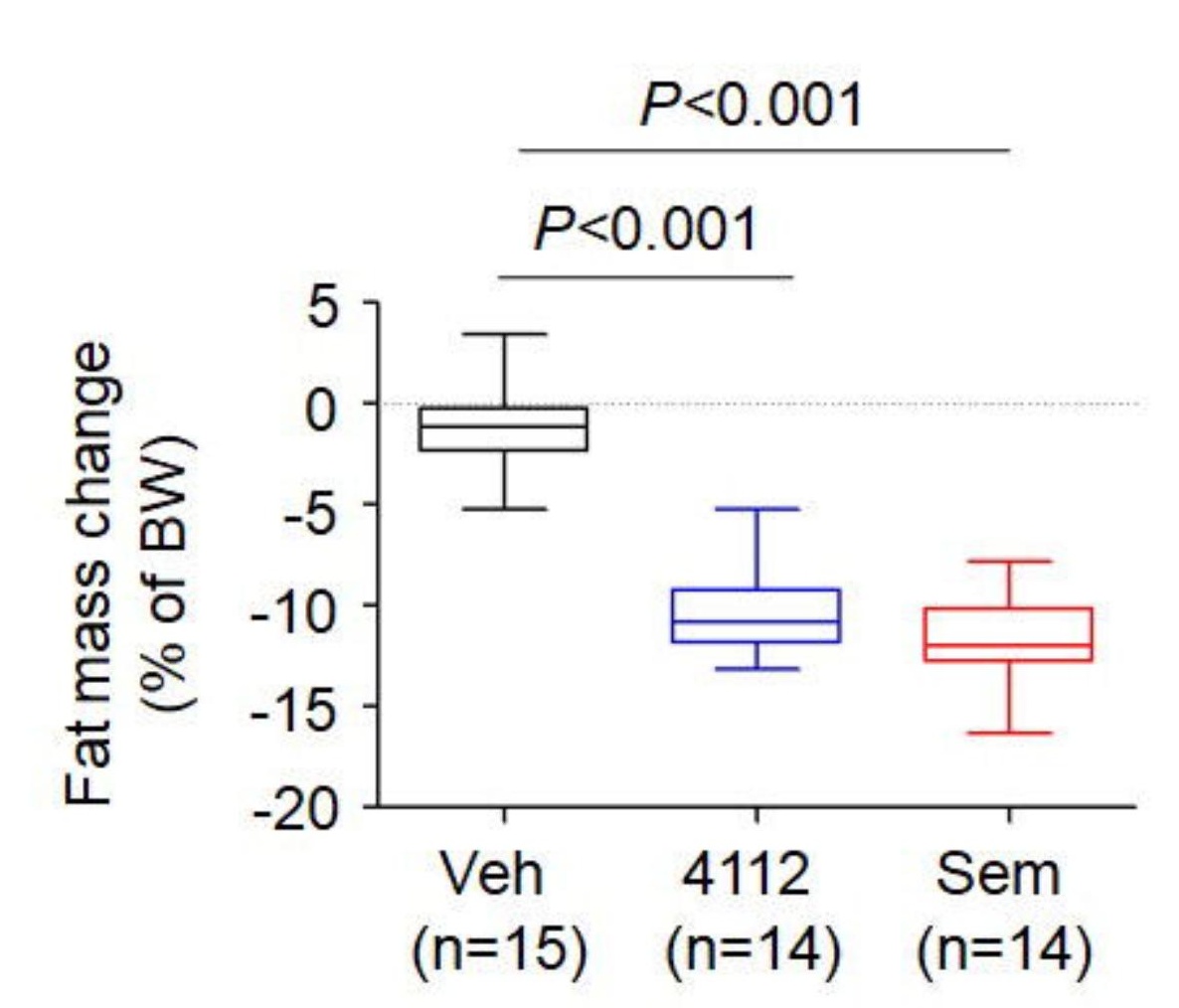

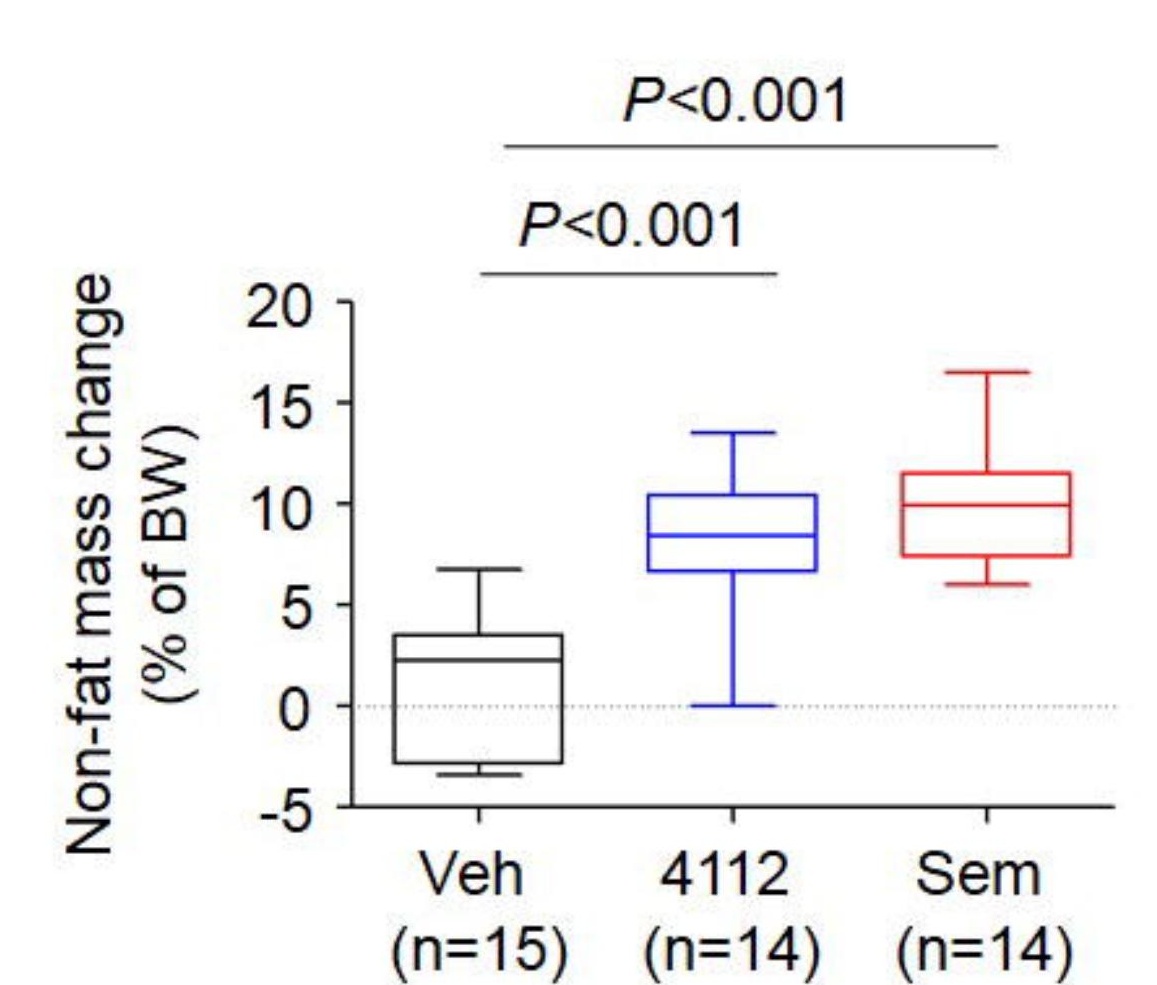

$\begin{array}{ccc}V e n & 4112 & \text { Sem } \\ (n=15) & (n=14) & (n=14)\end{array}$

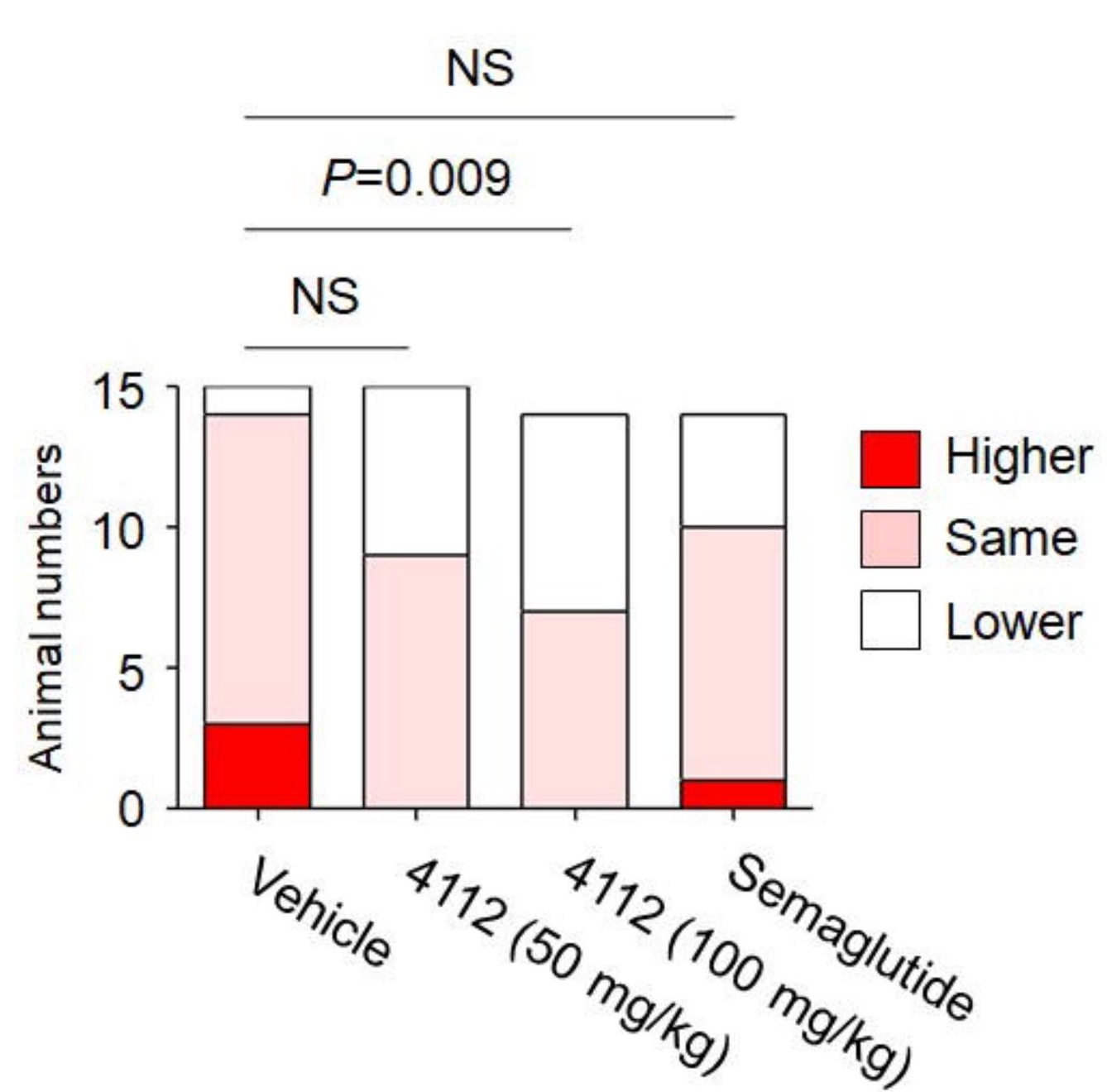

h

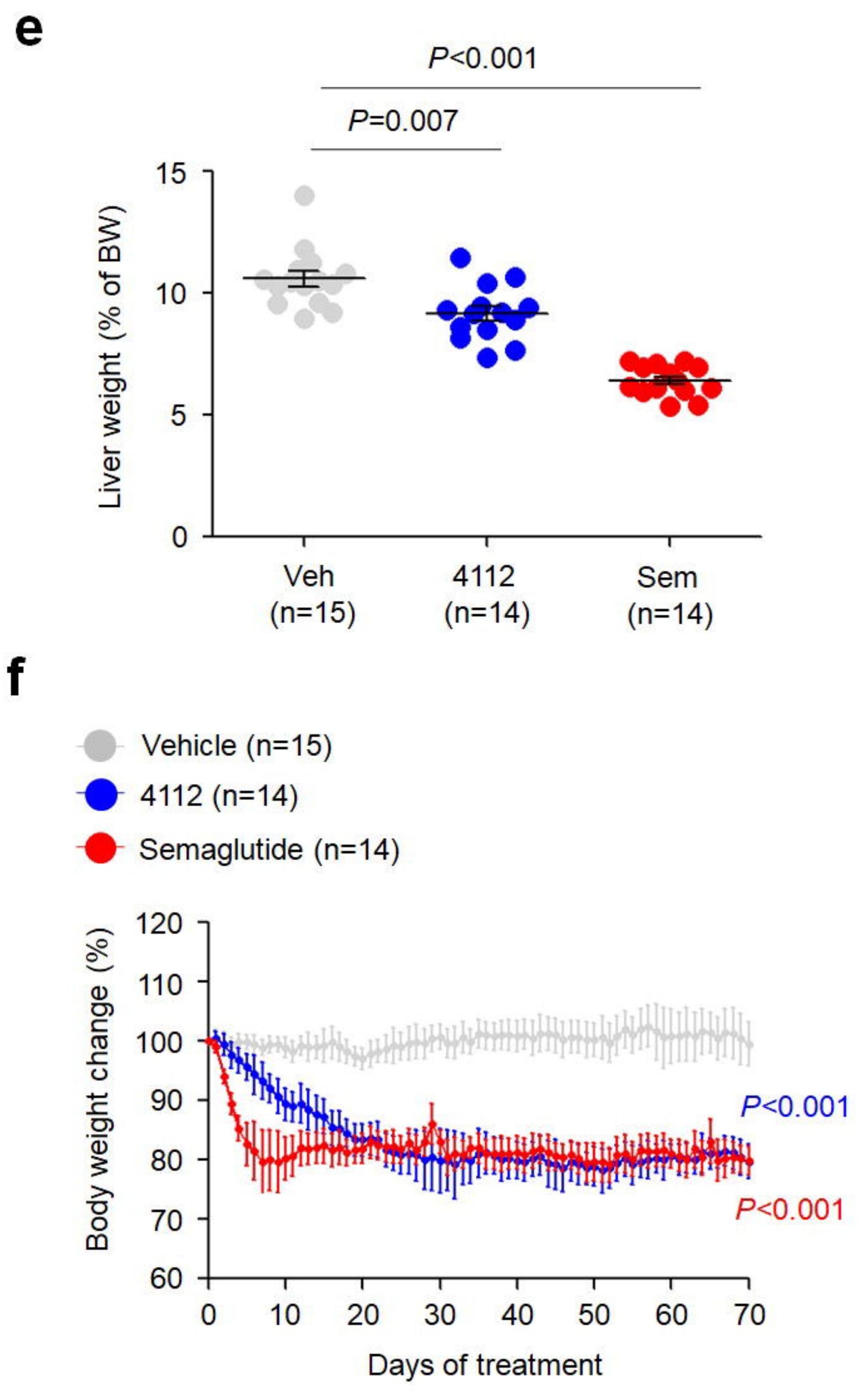

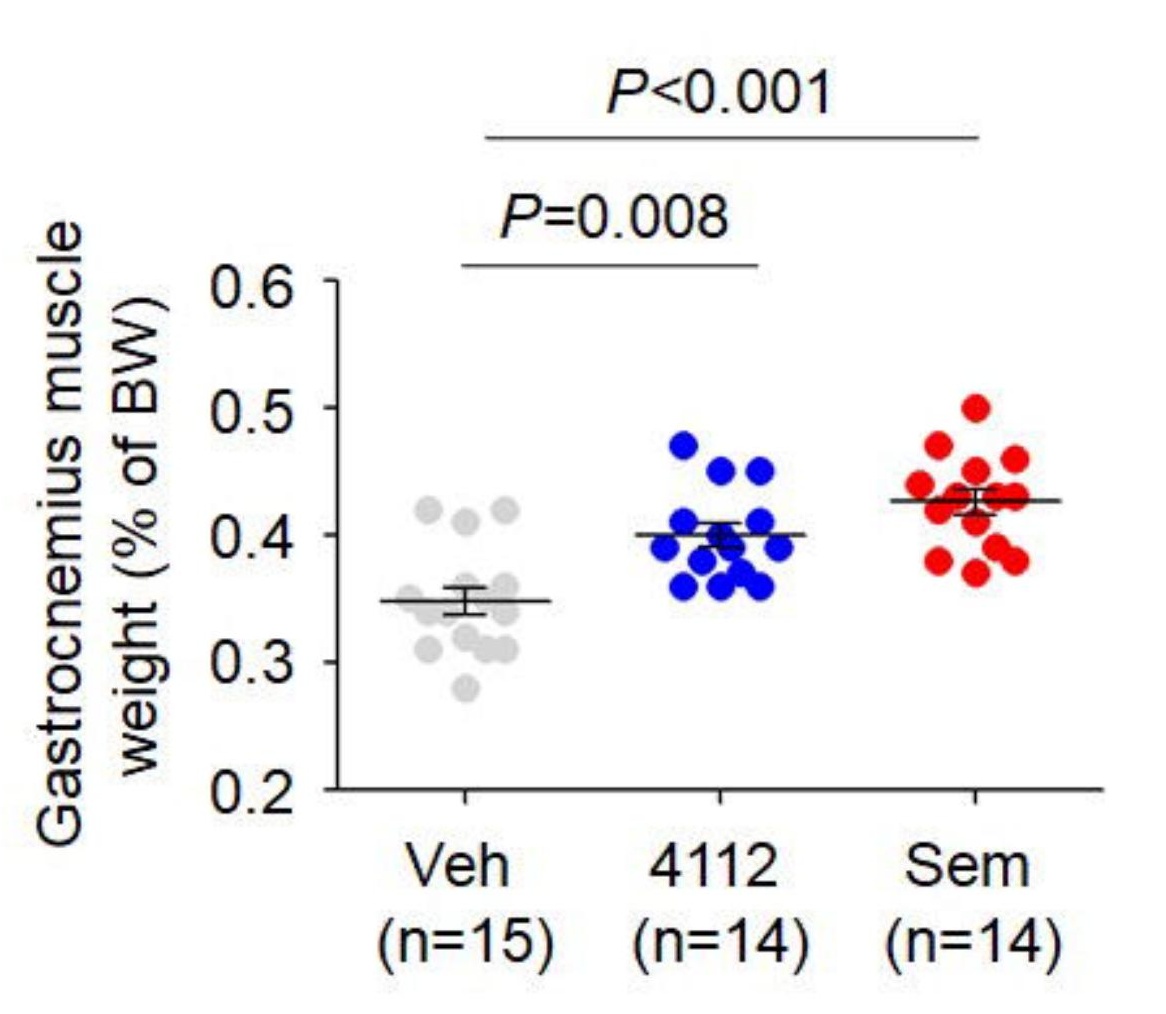

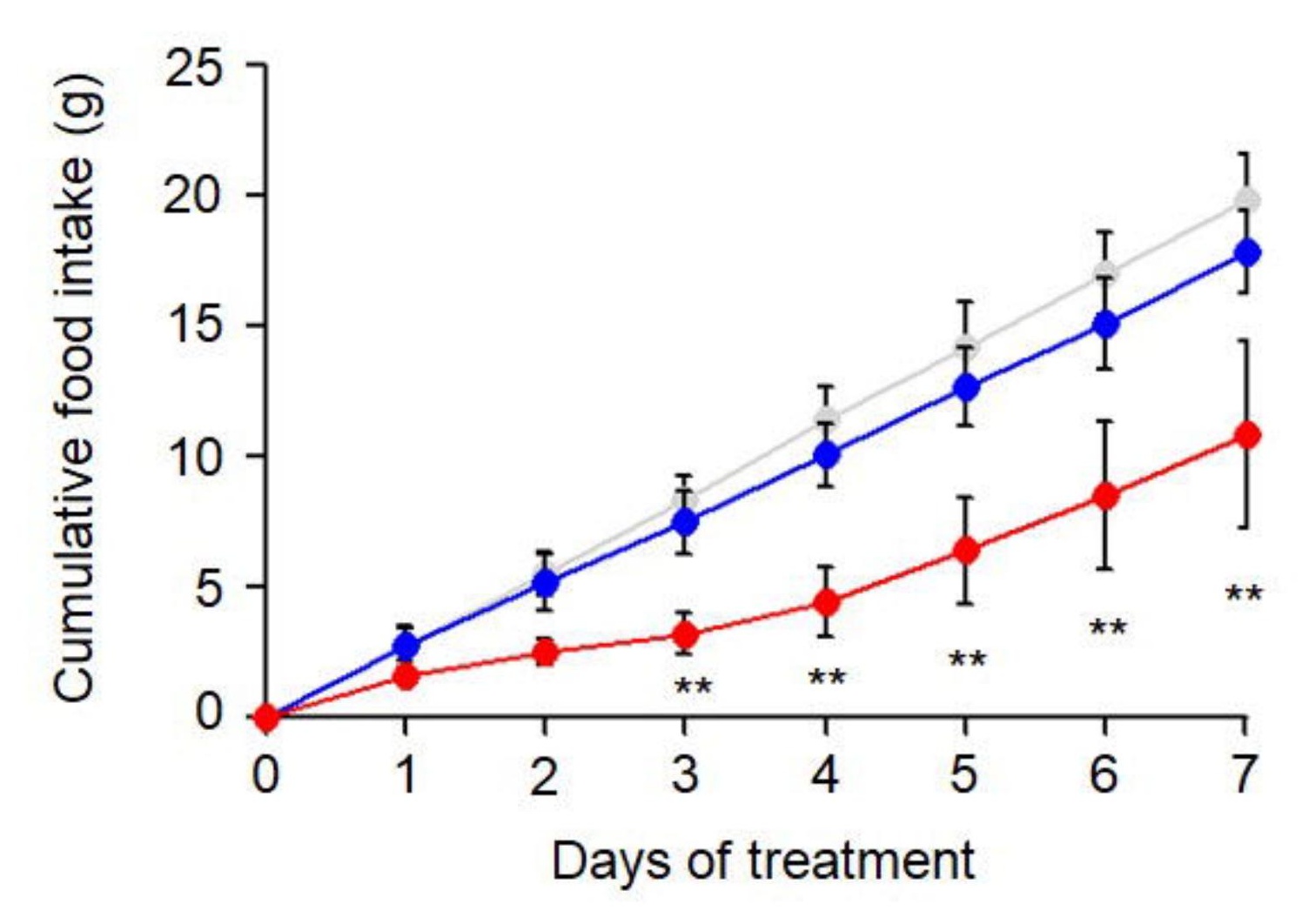


a

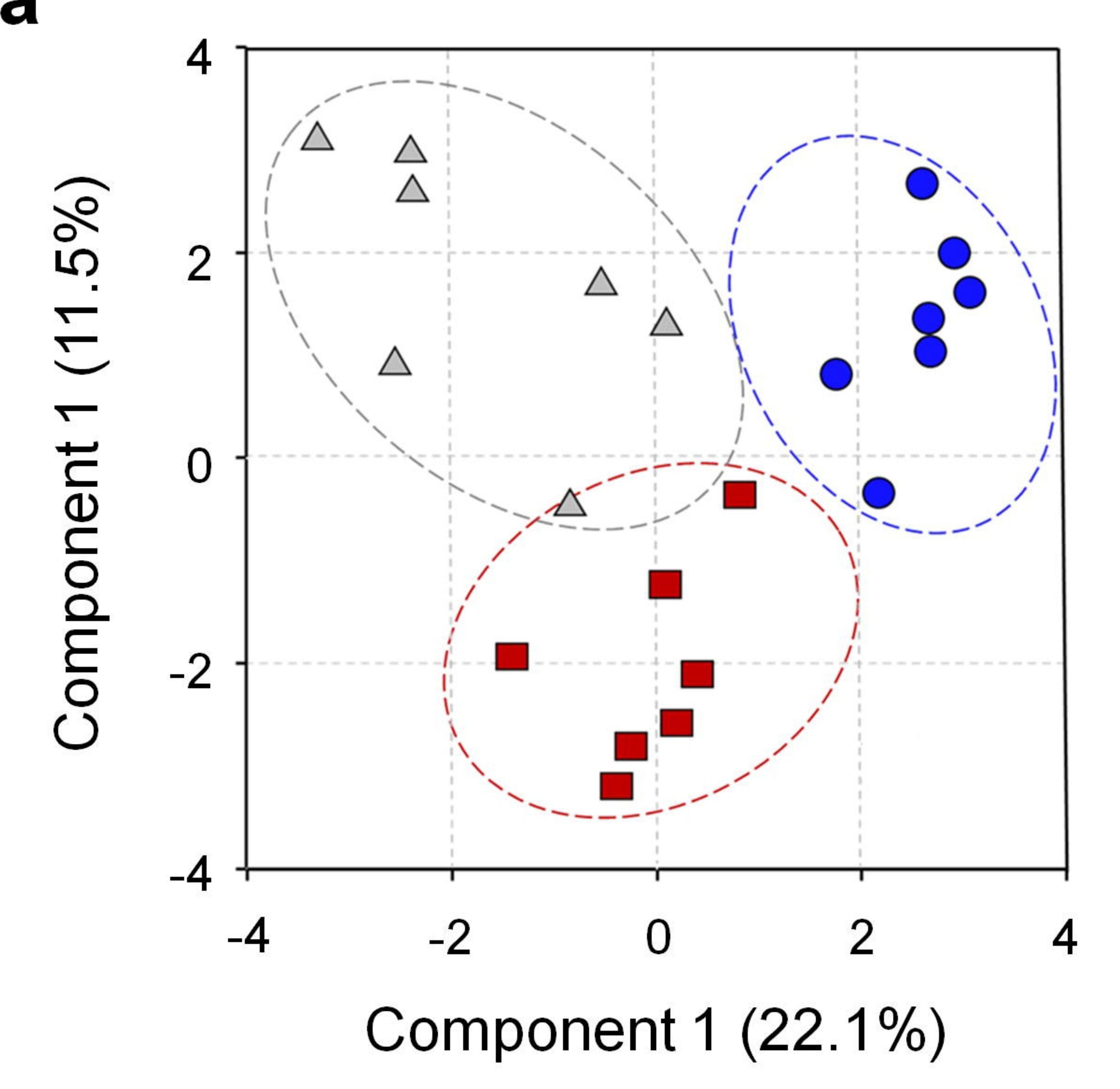

$\triangle$ Vehicle

- 4112

- Semaglutide

C
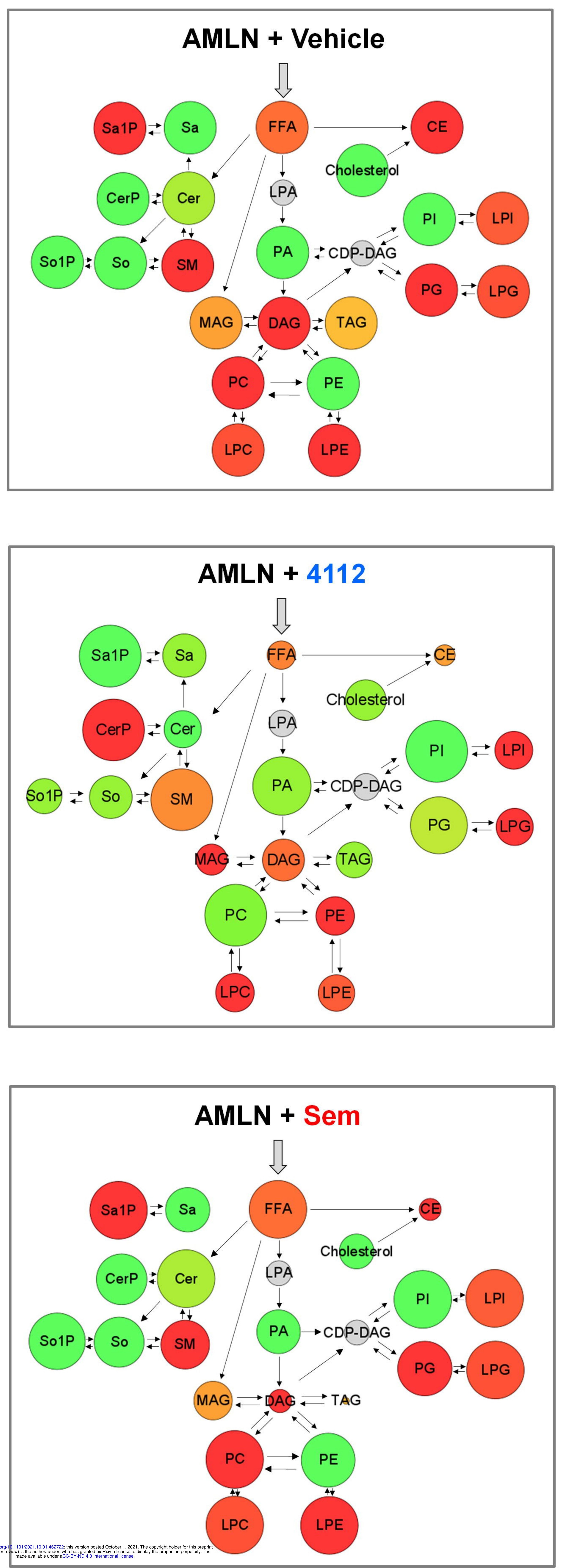

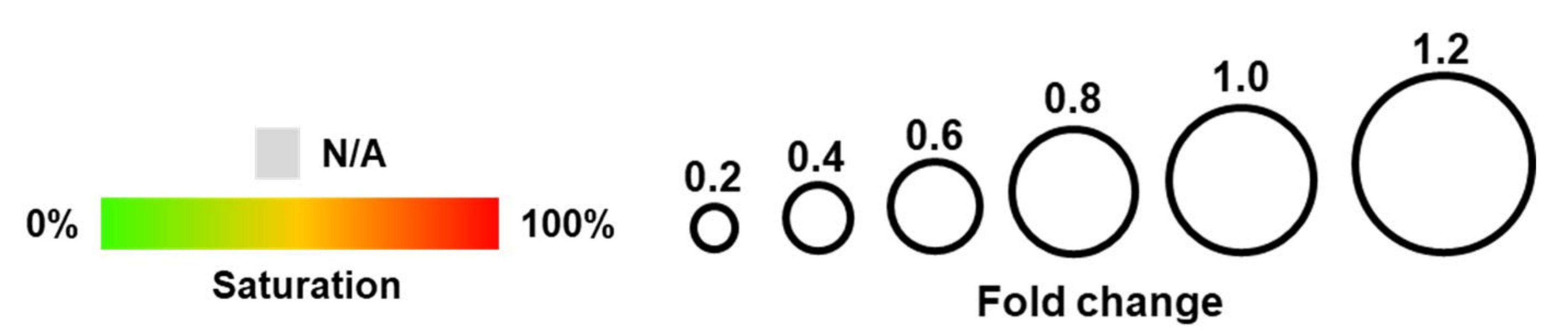

b
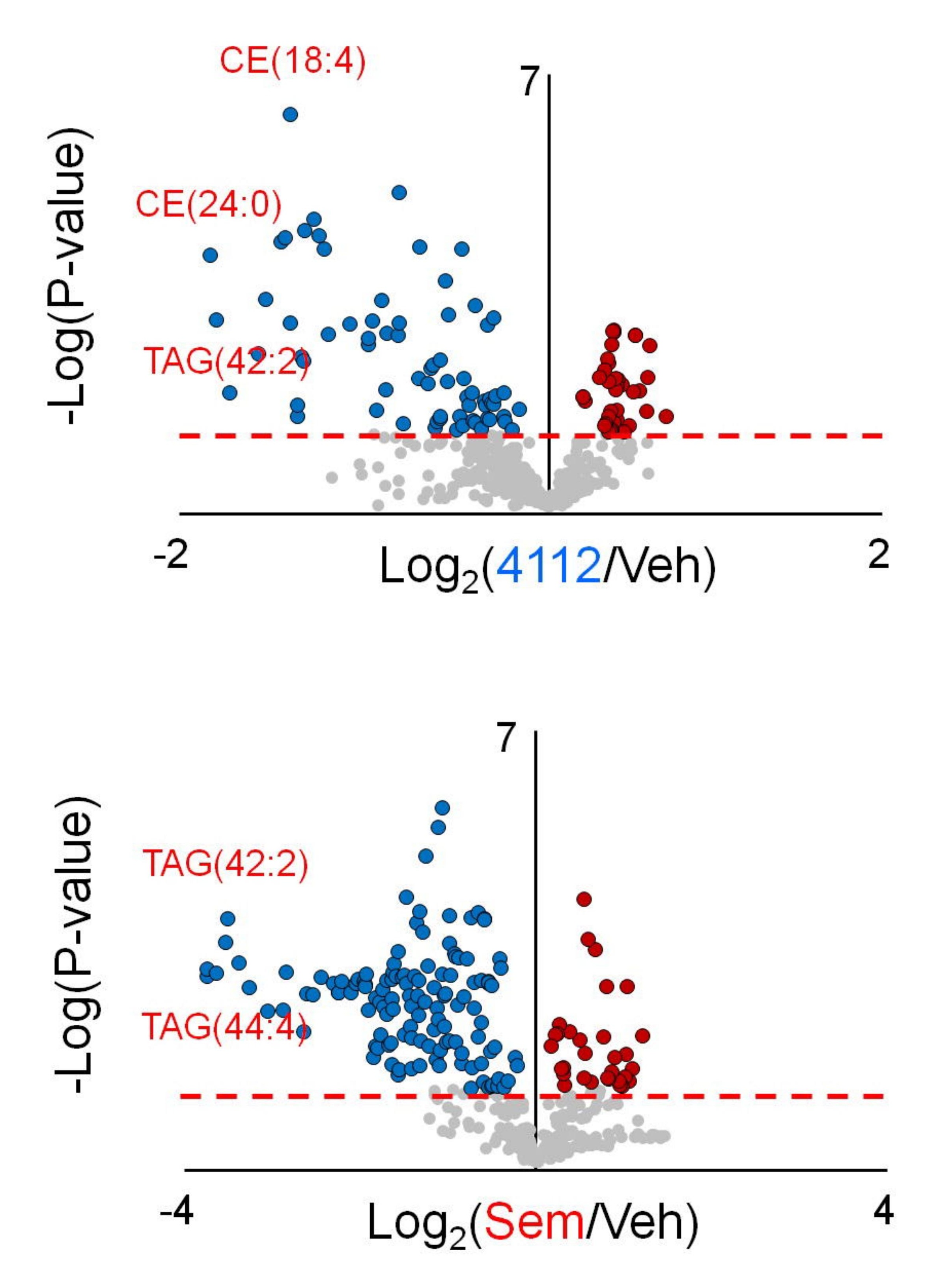

d

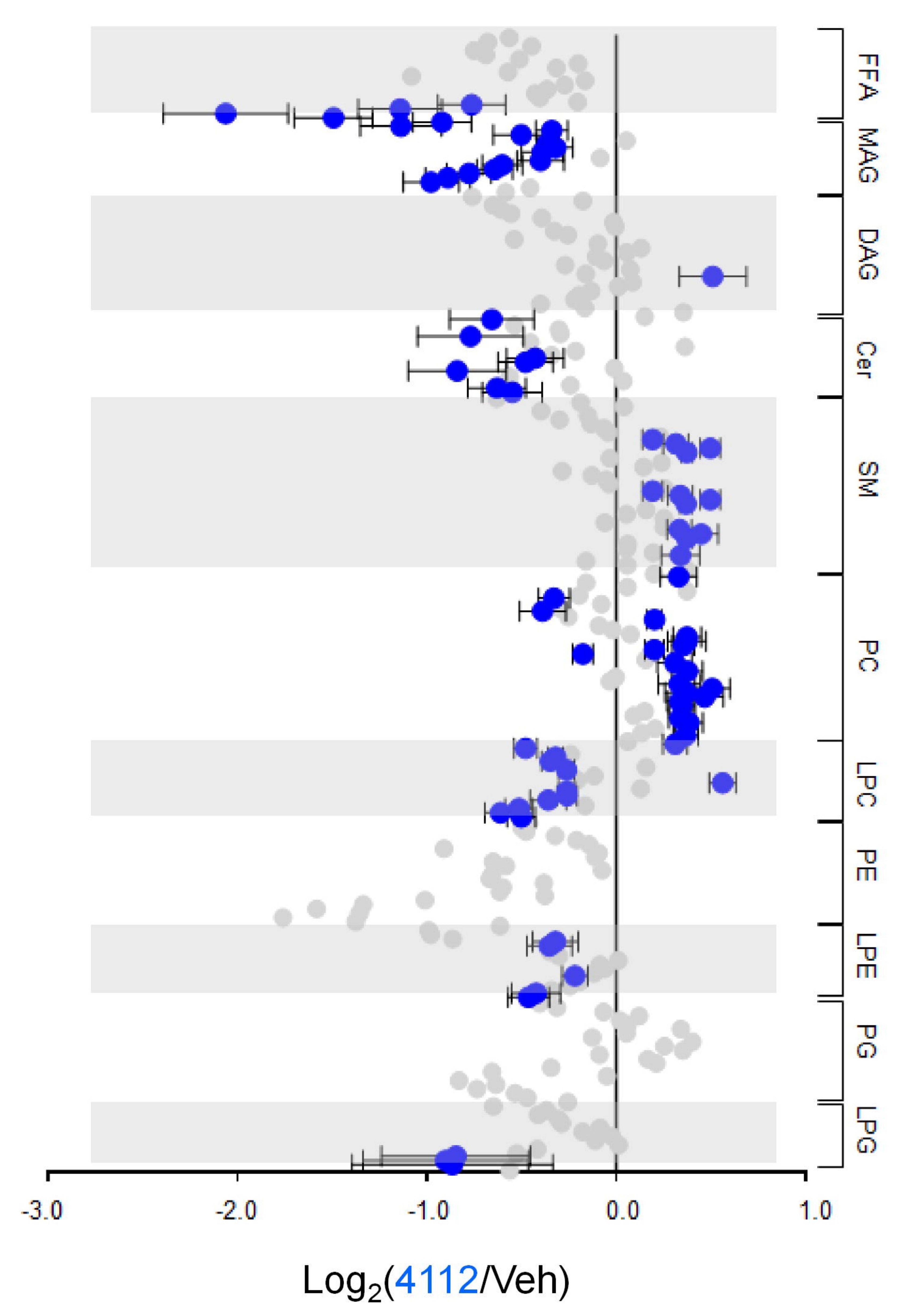

e
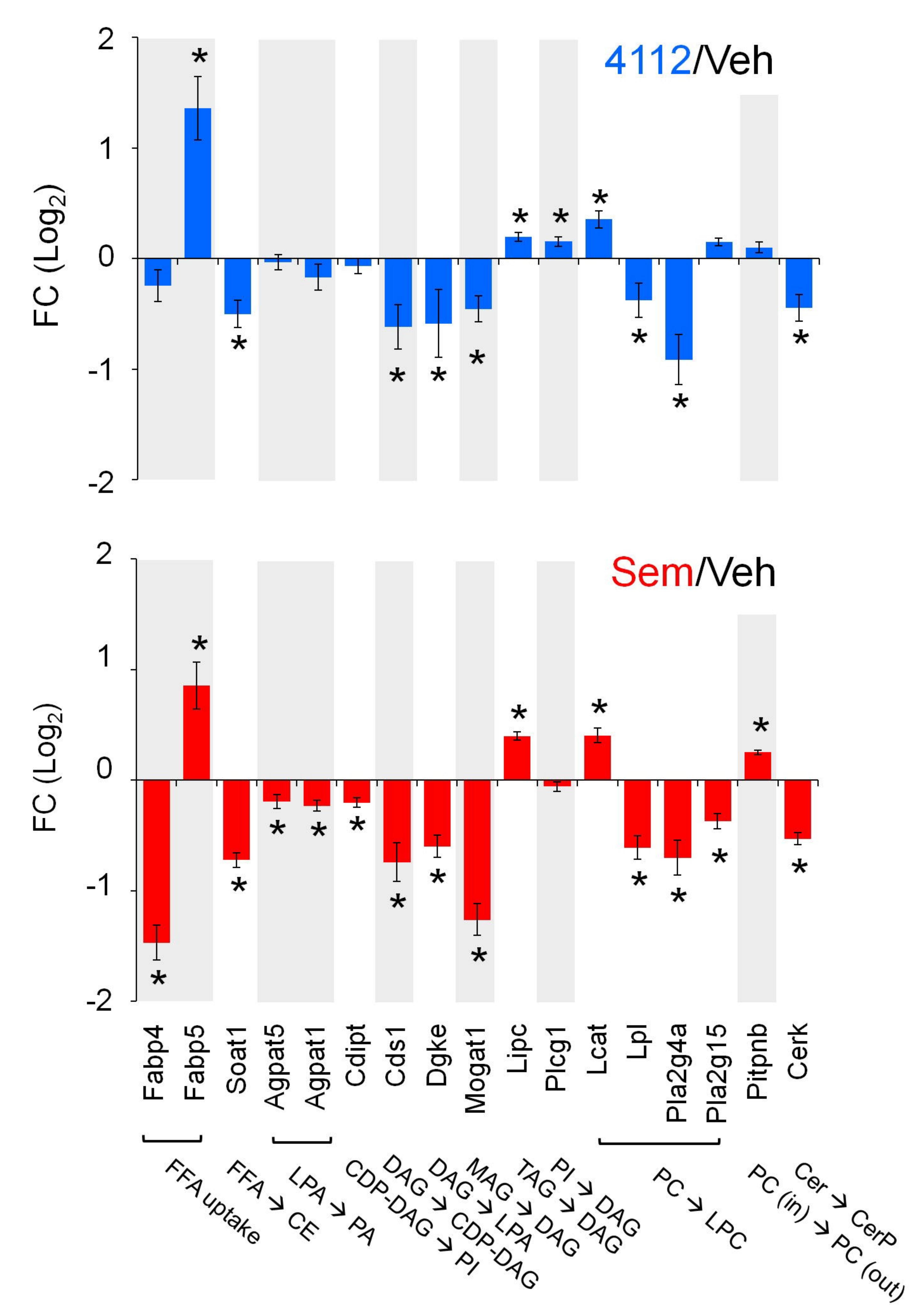

UP
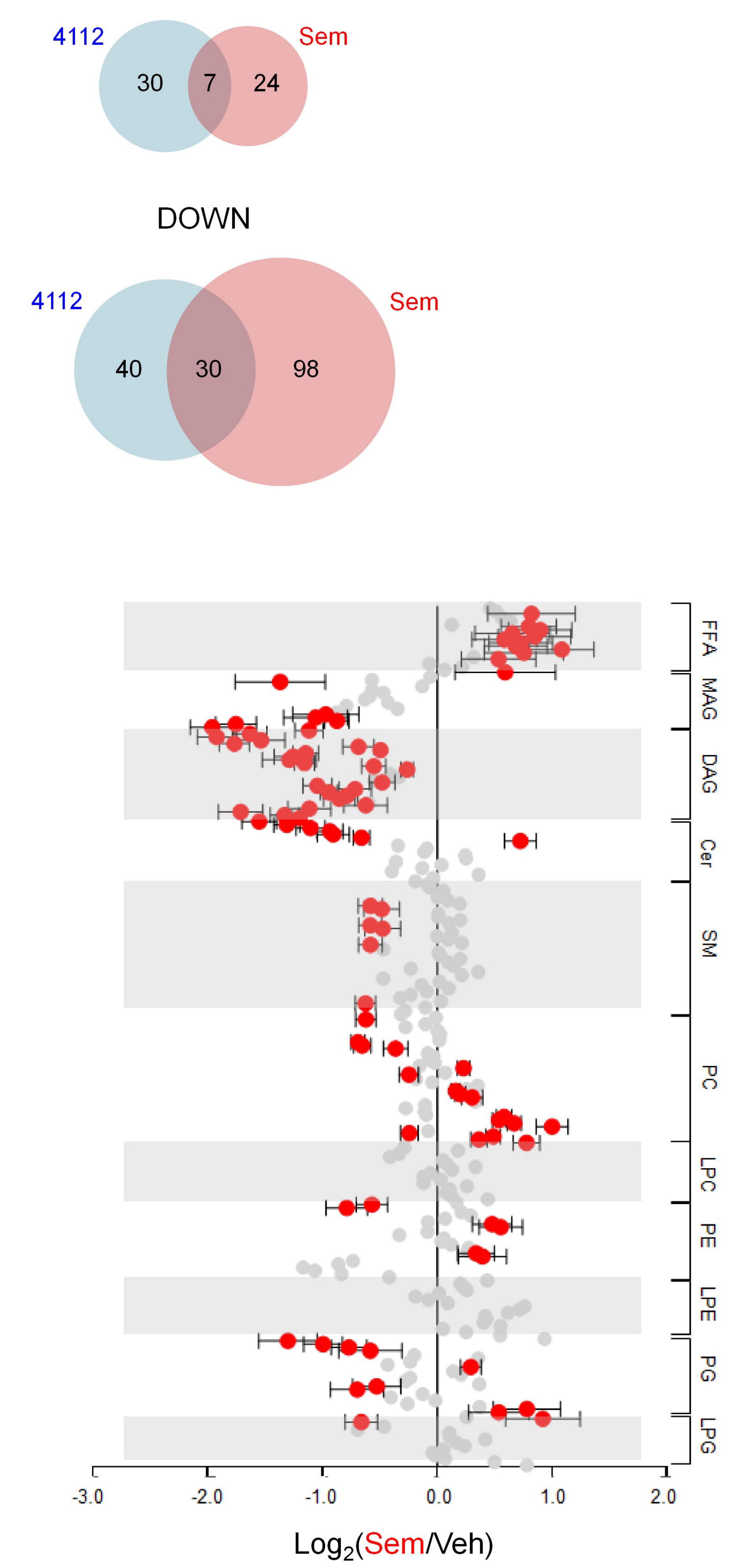

f
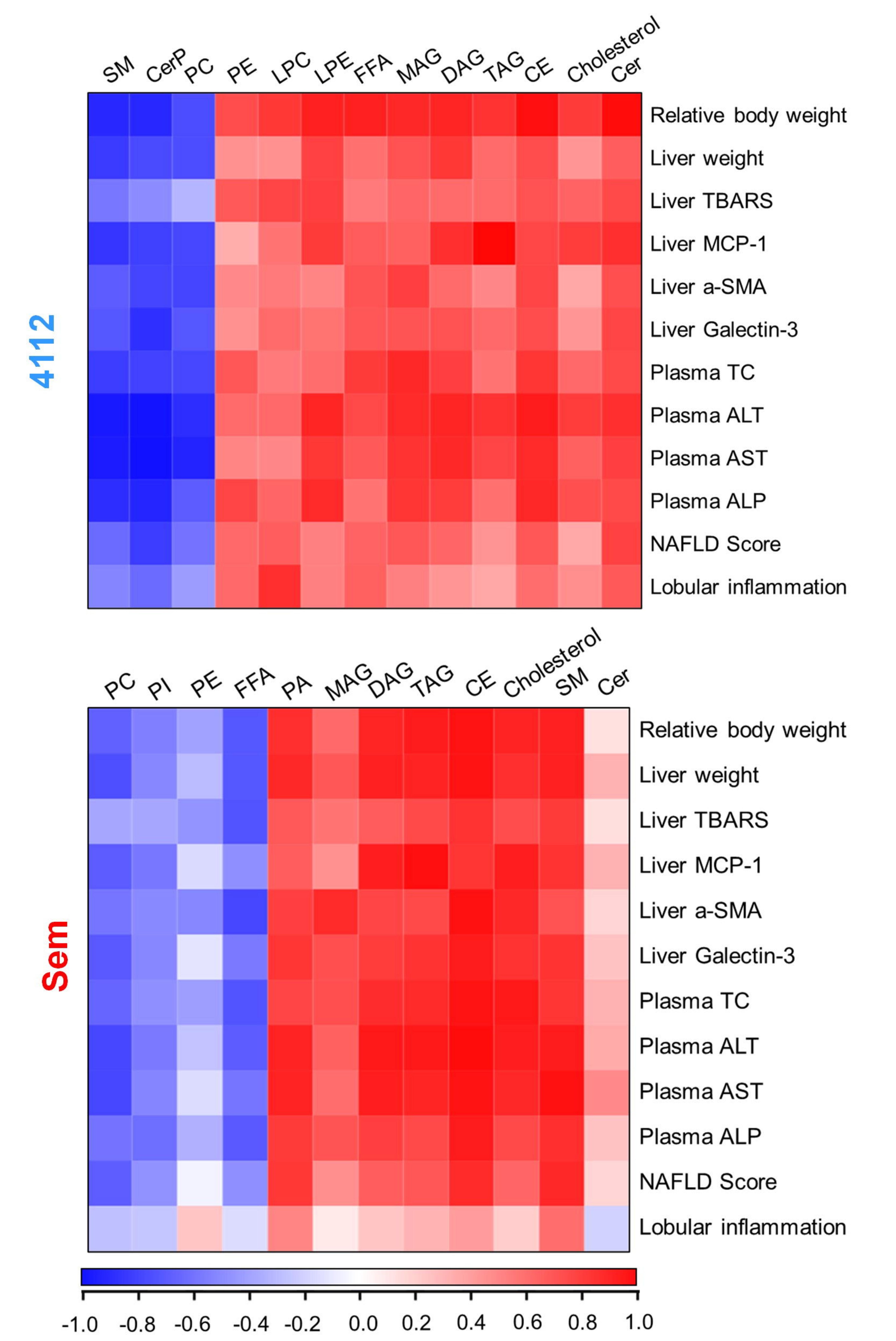

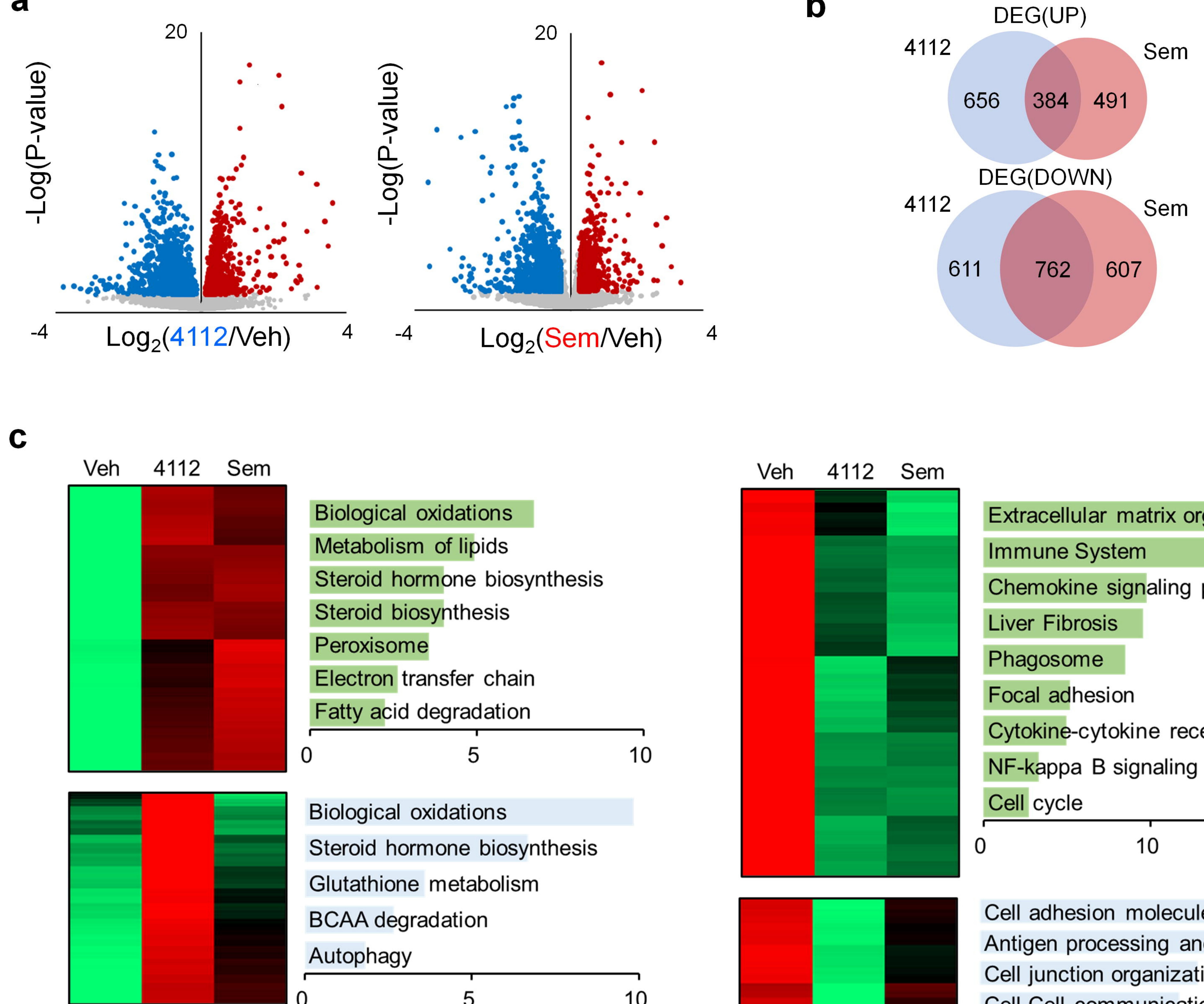

Biological oxidations

Metabolism of lipids

Steroid hormone biosynthesis

Steroid biosynthesis

Peroxisome

Electron transfer chain

Fatty acid degradation

$\stackrel{5}{0}$

Biological oxidations Steroid hormone biosynthesis

Glutathione metabolism

BCAA degradation

Autophagy
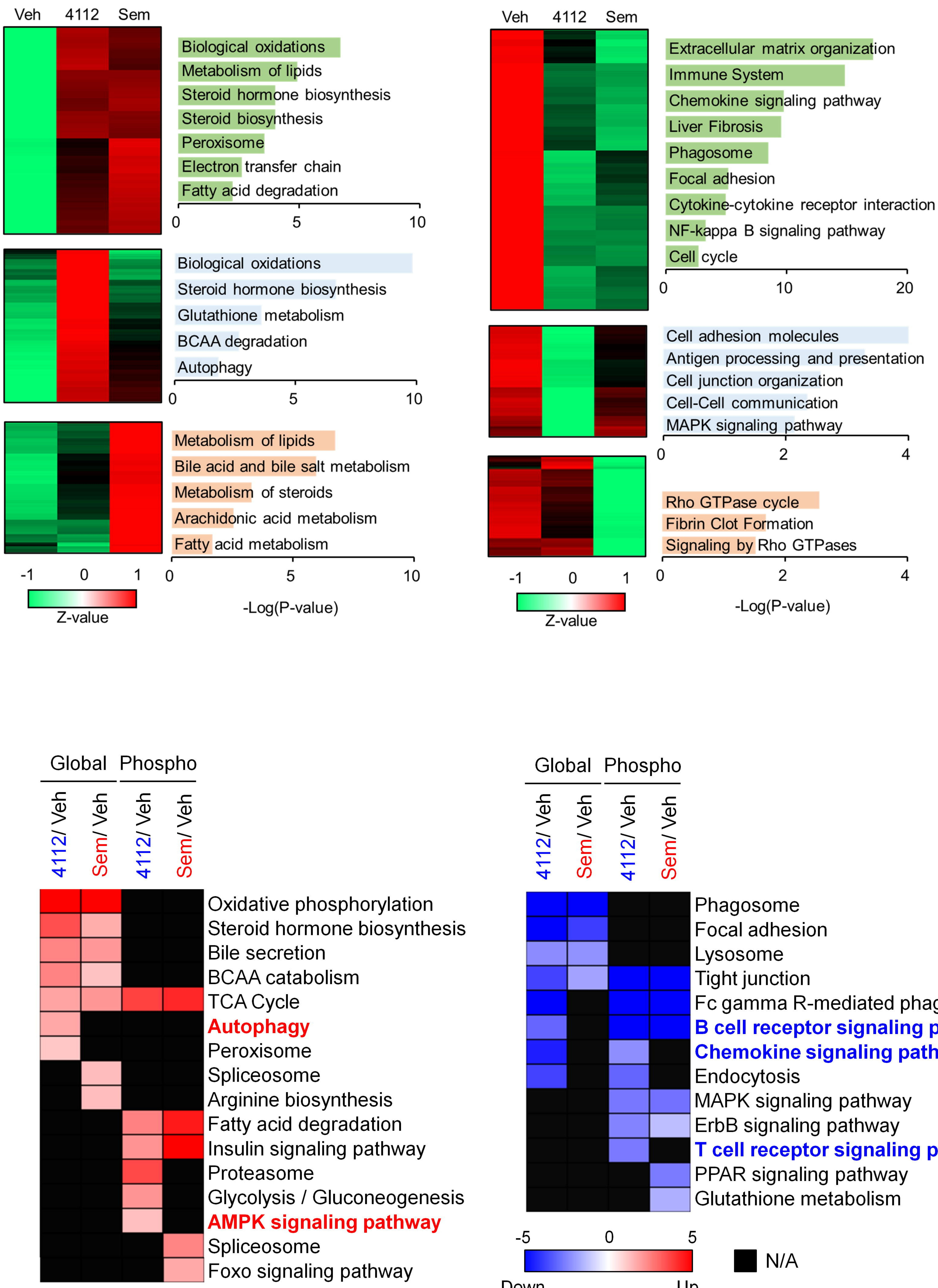

Insulin receptor signaling pathway

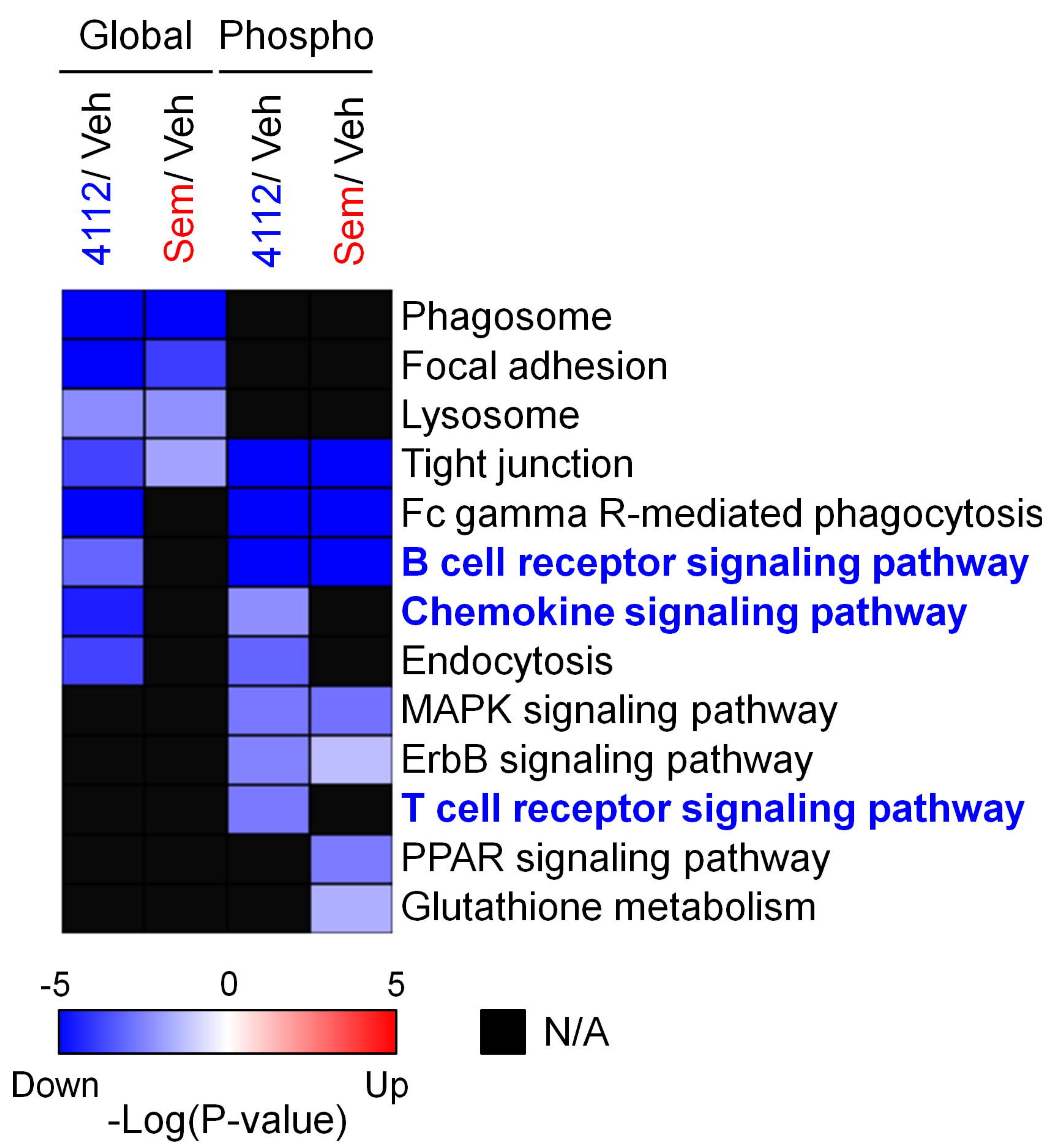

oxidative stress response

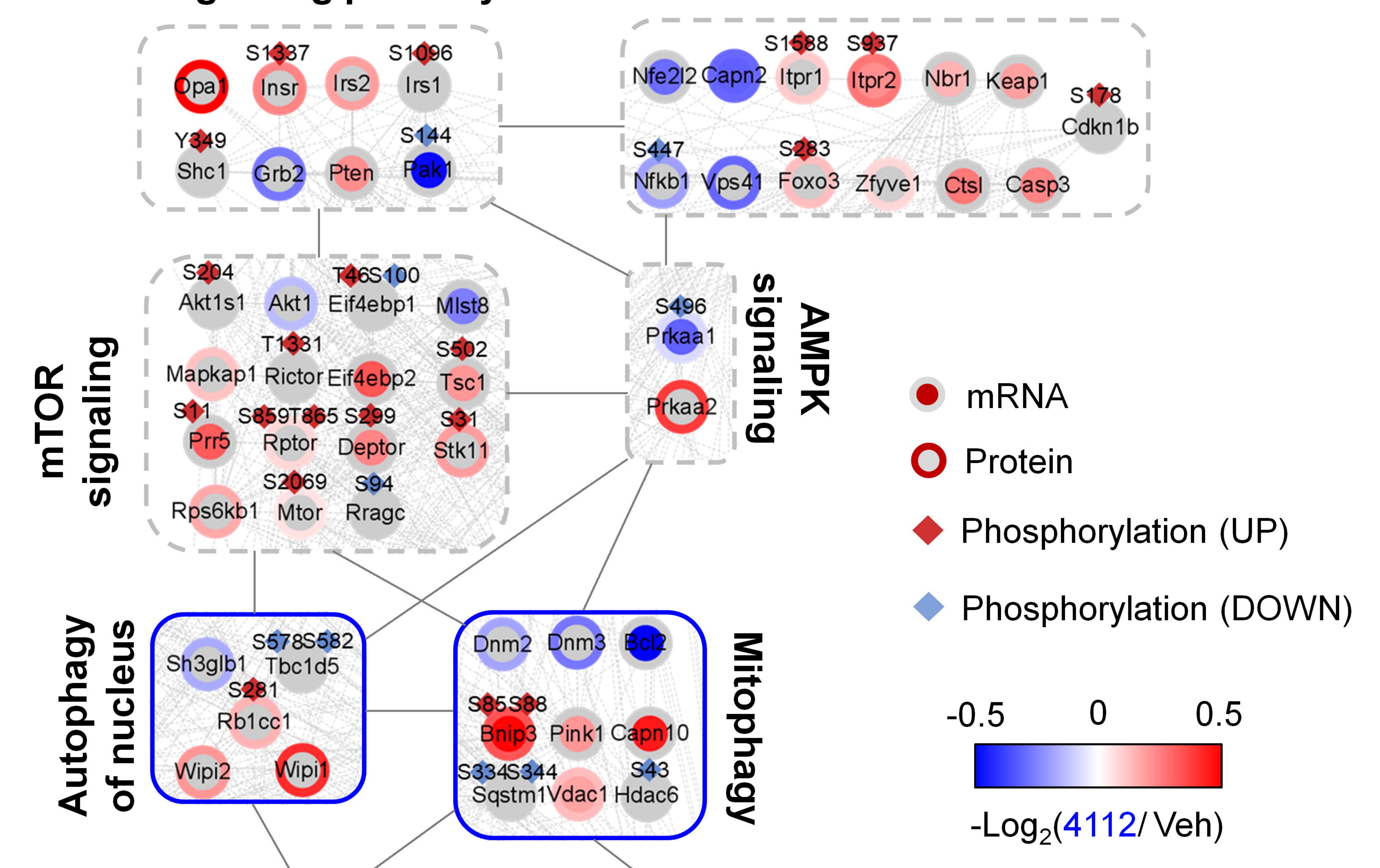

Ulk2 UIk

Atg101

Lrsam1 Atg14 Atg5
S389 5875 S14
Atg2b Atg3 Atg2a Atg9a

Autophagosome assembly

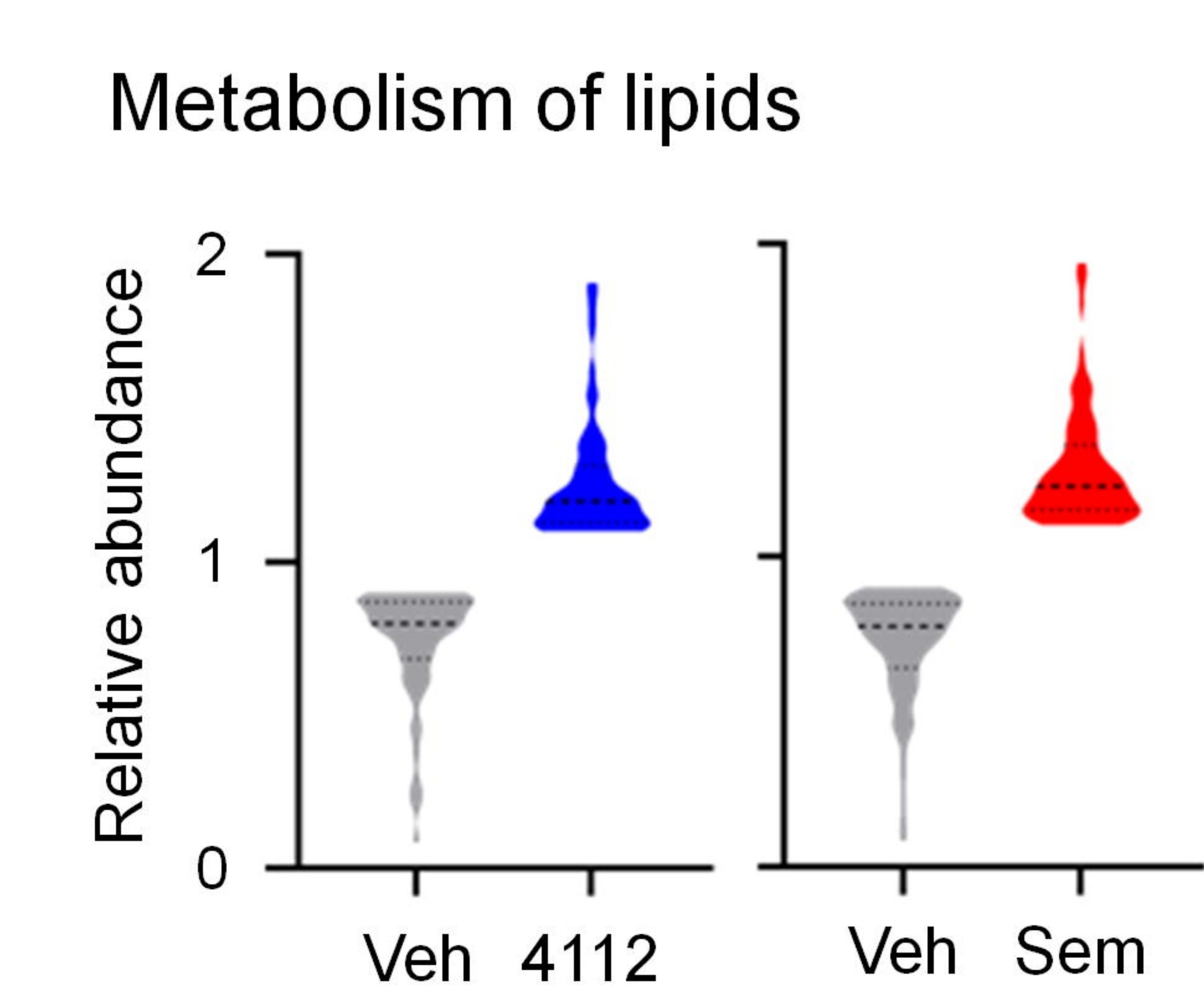

Extracellular matrix organization
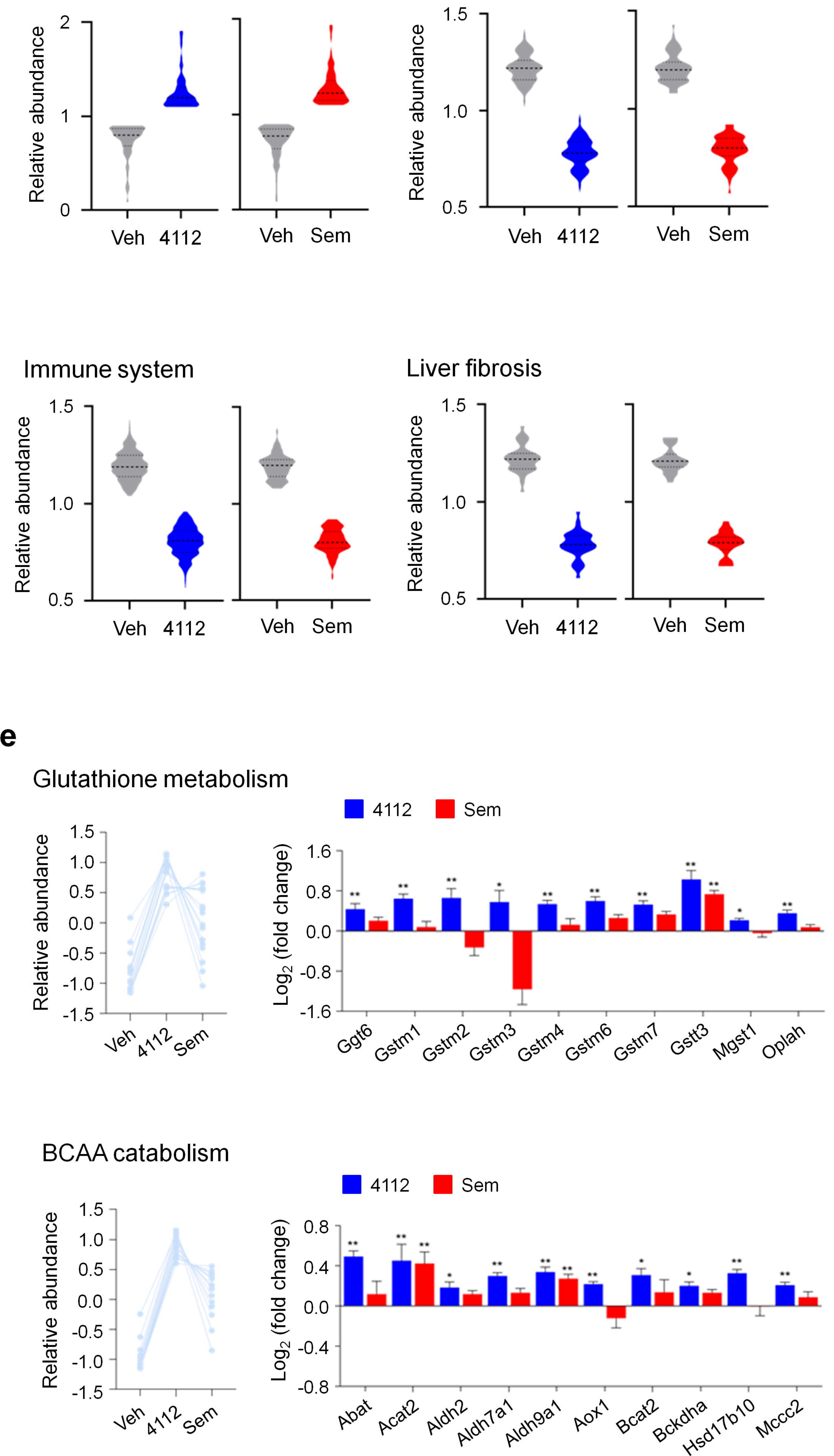

Autophagy
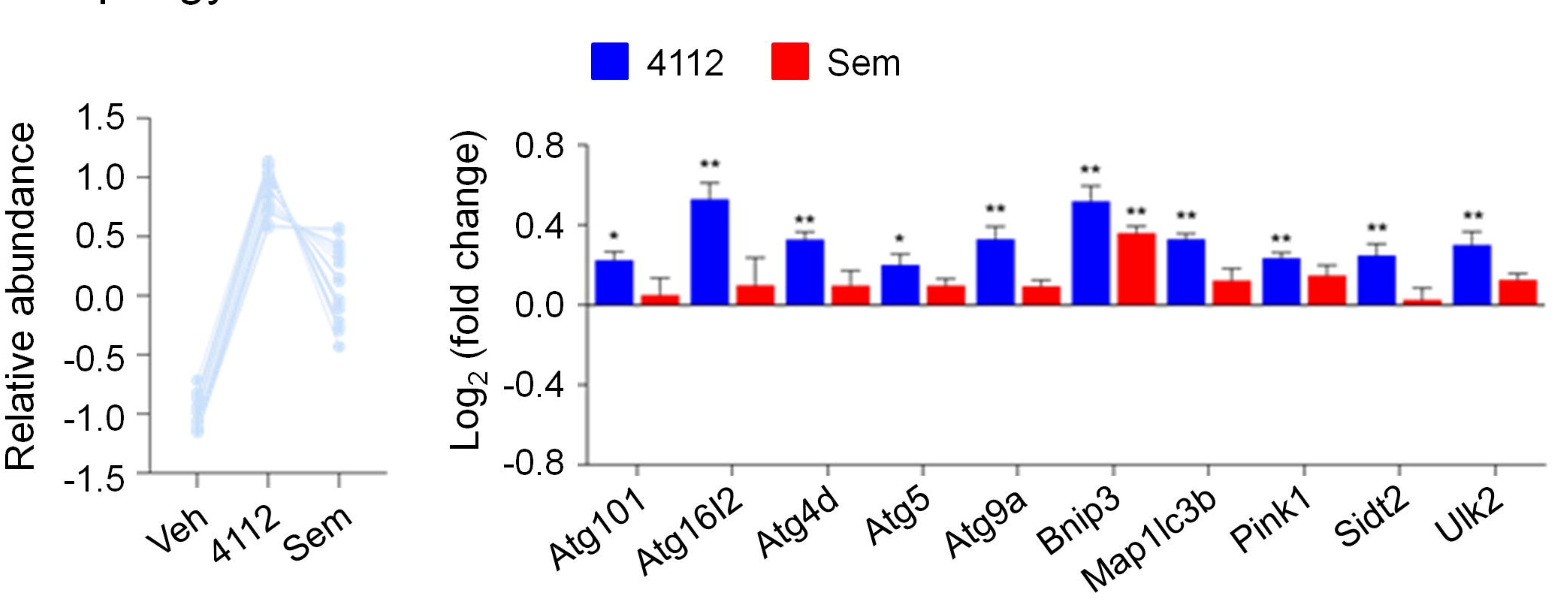

Cell adhesion molecules

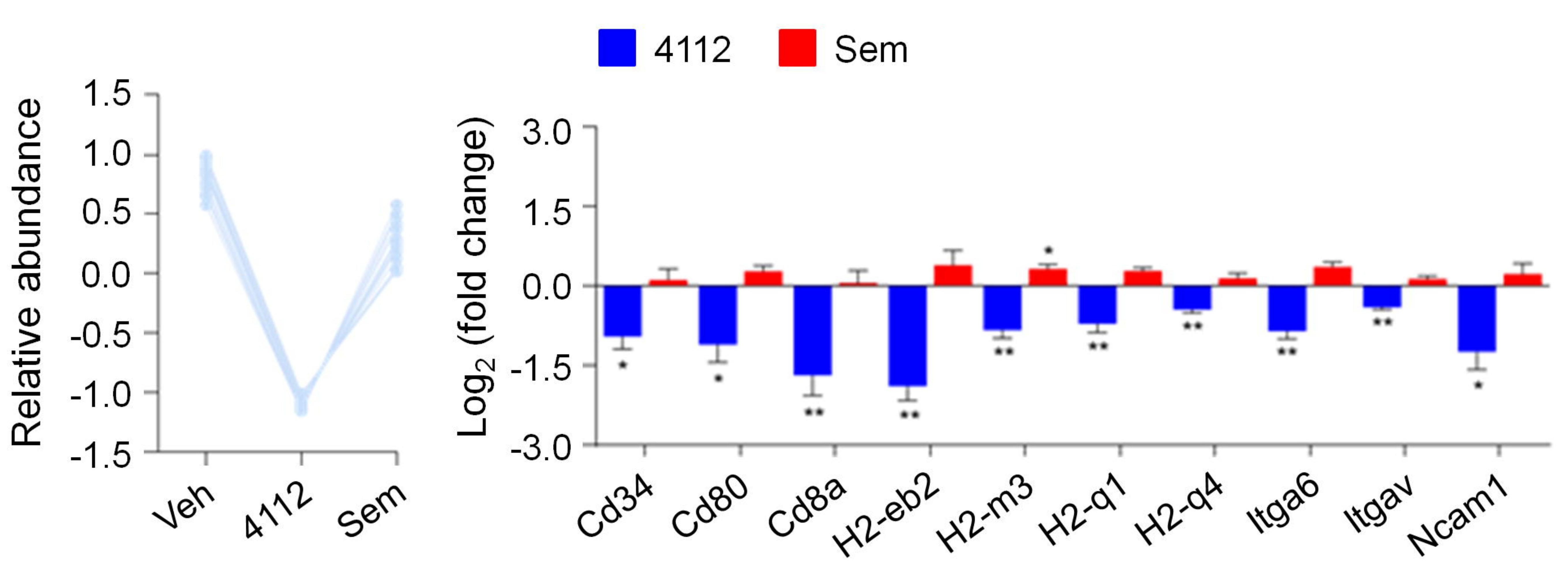

Antigen processing and presentation
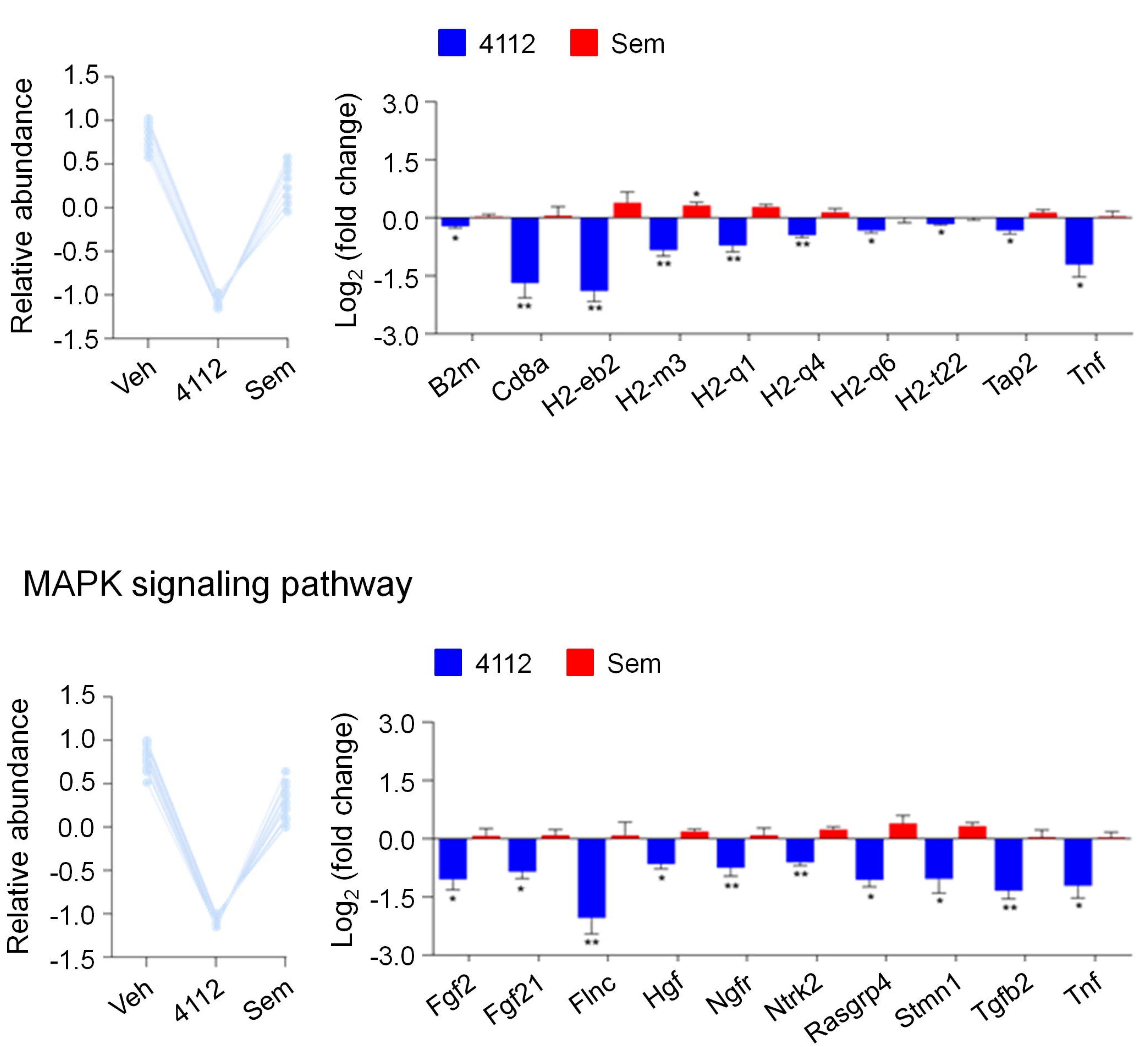

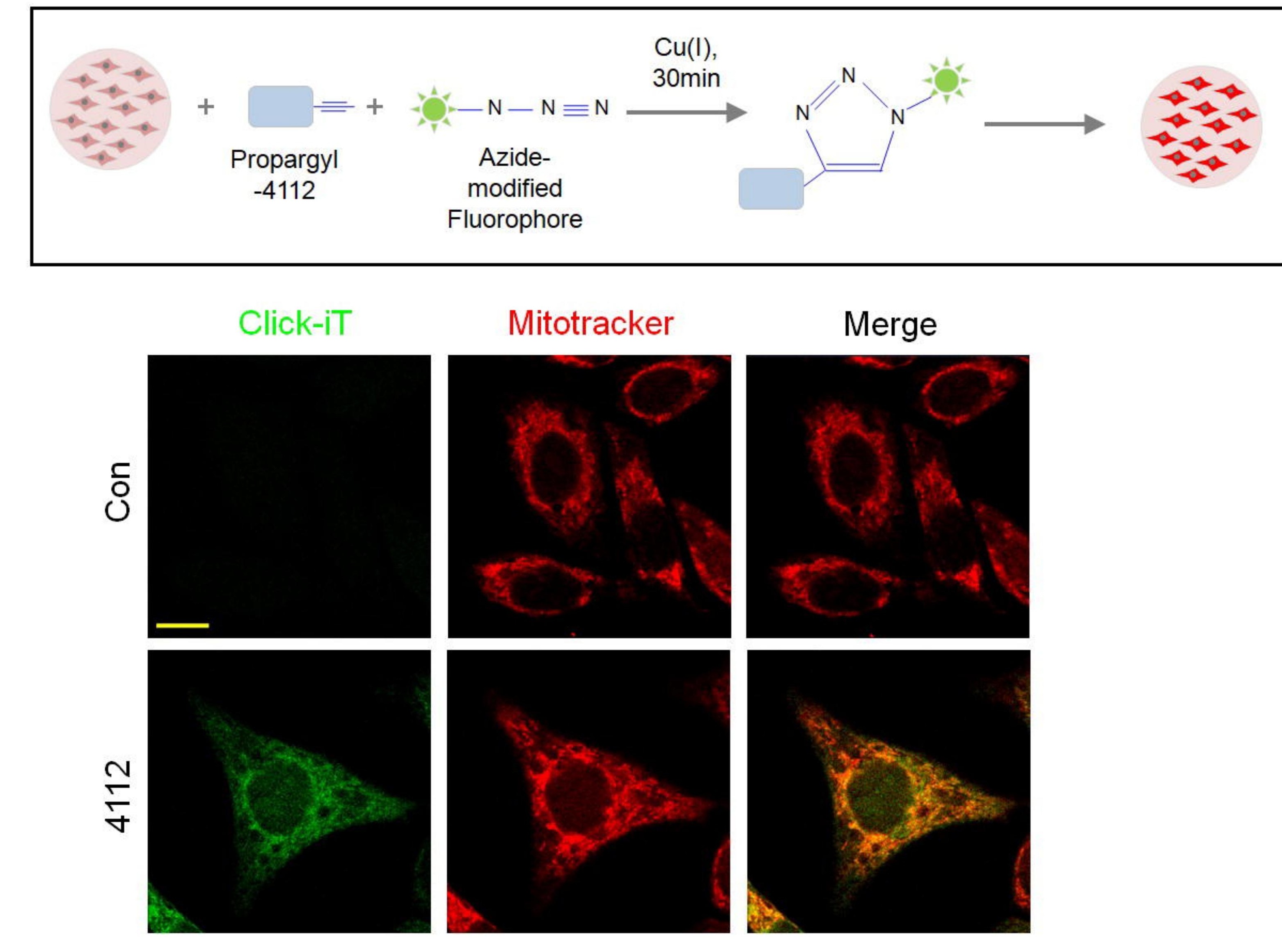

b
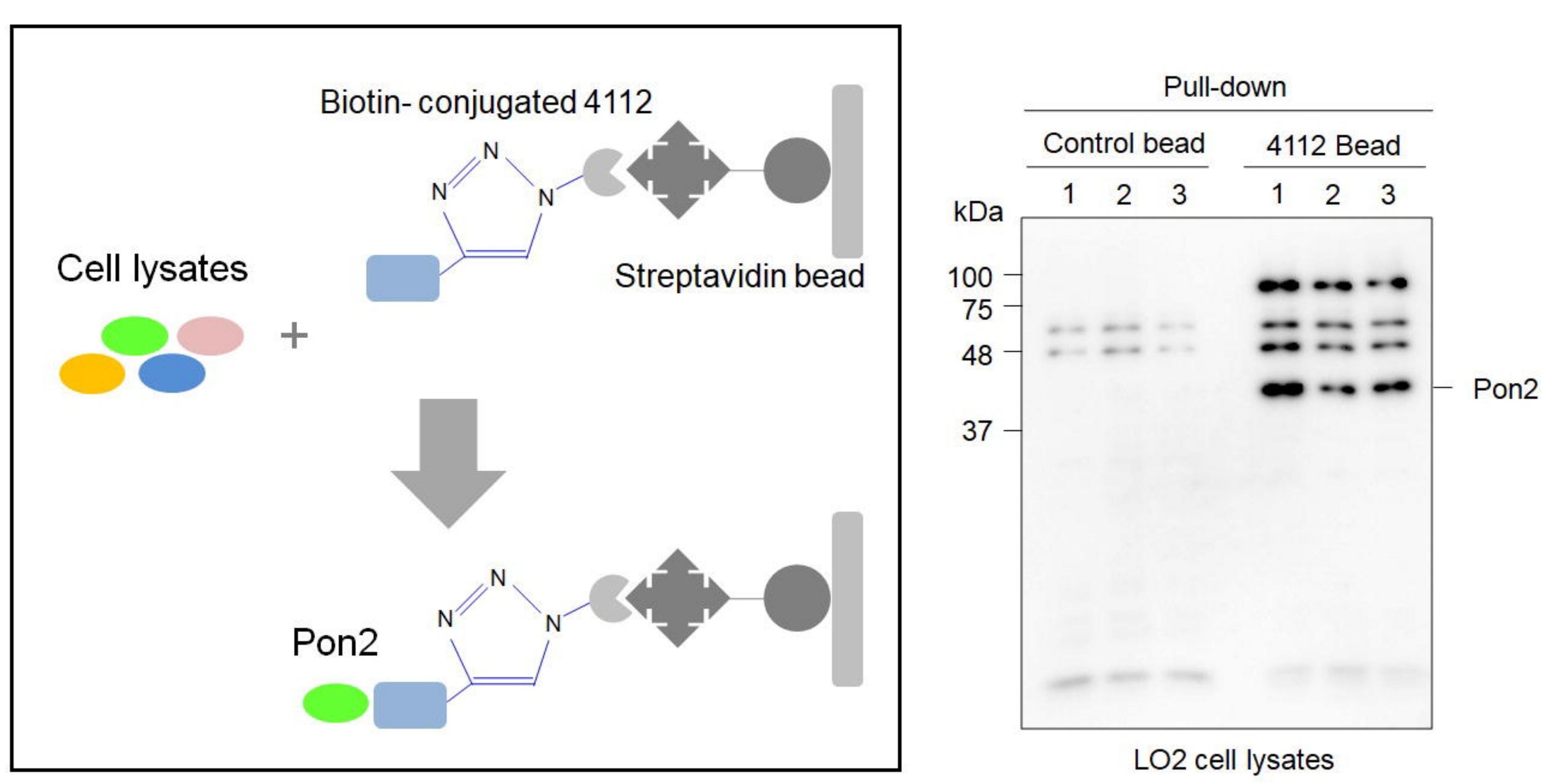

c
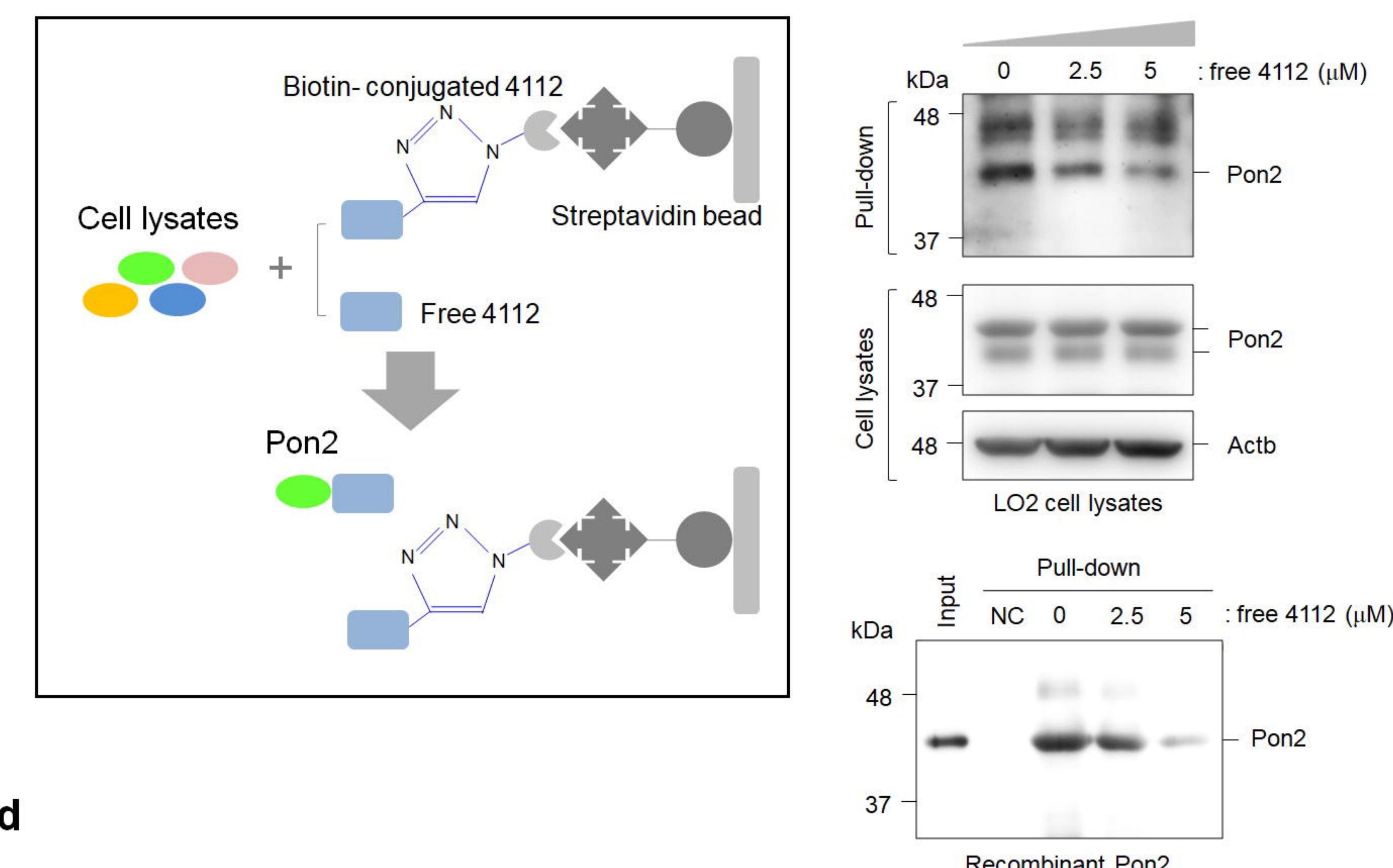

In L02 cells

- $\mathrm{Co}$

DA $\mathrm{PA}+4112$

$$
\text { 일 }
$$

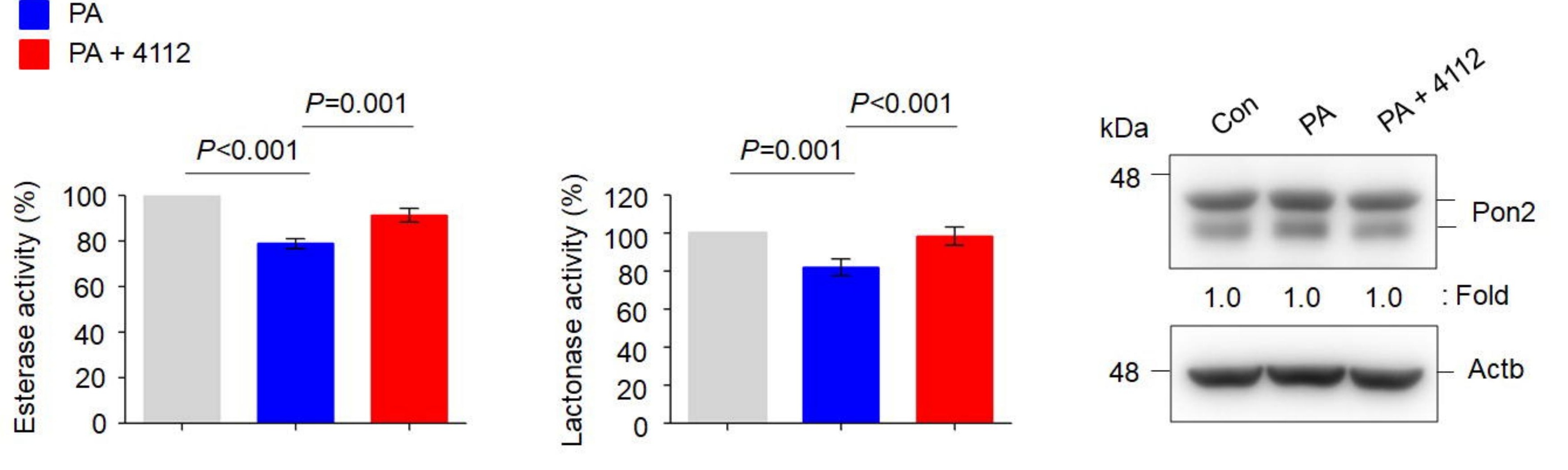

e

Con

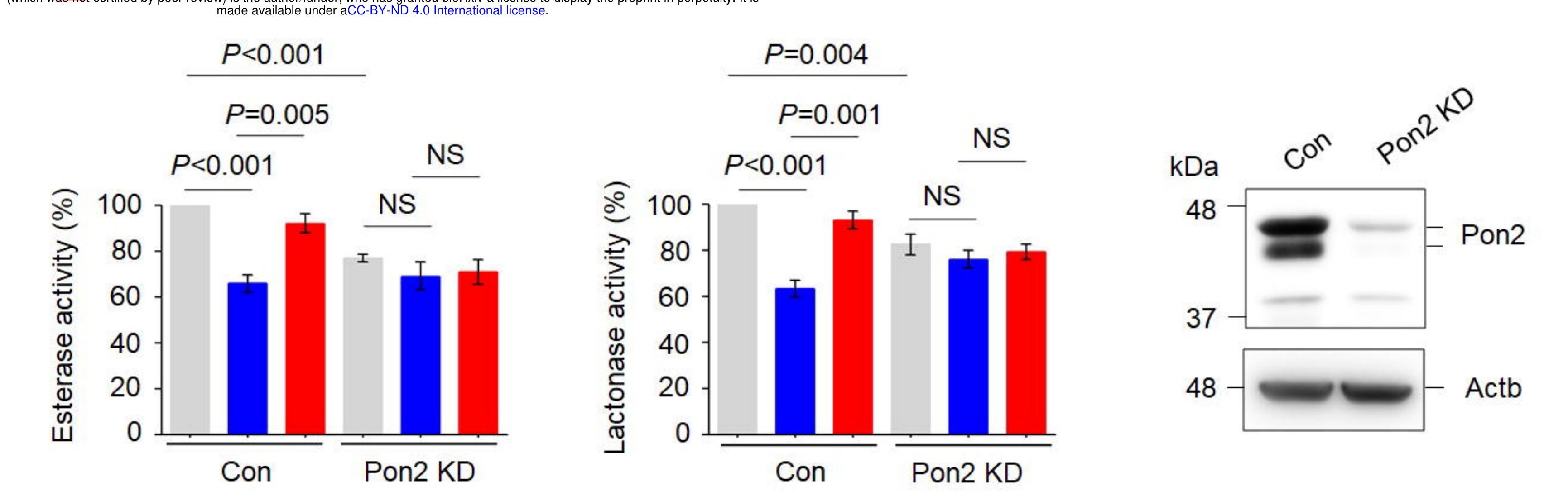

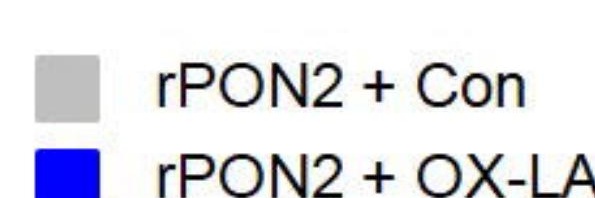

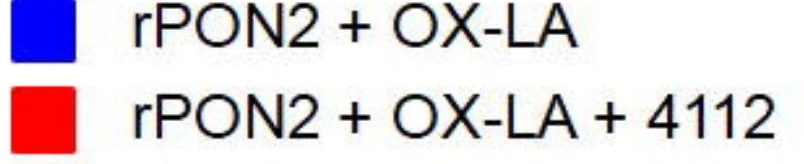

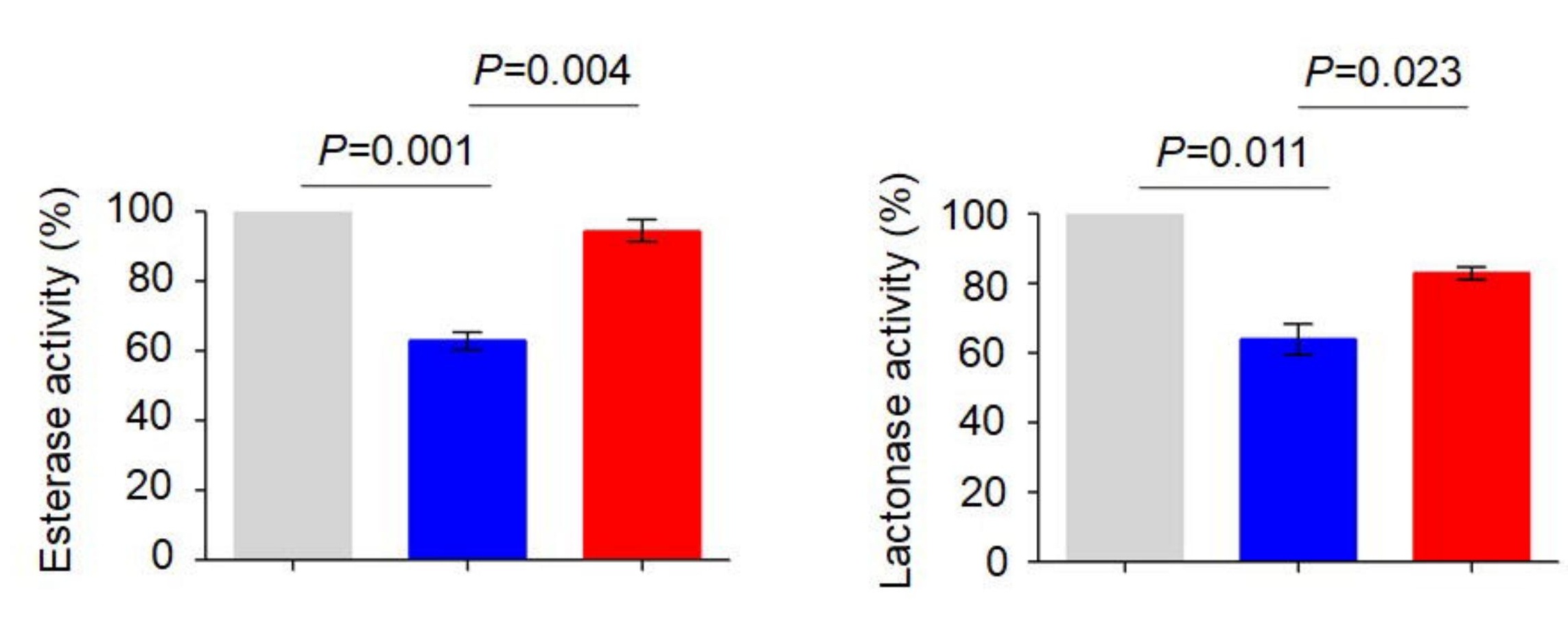

g
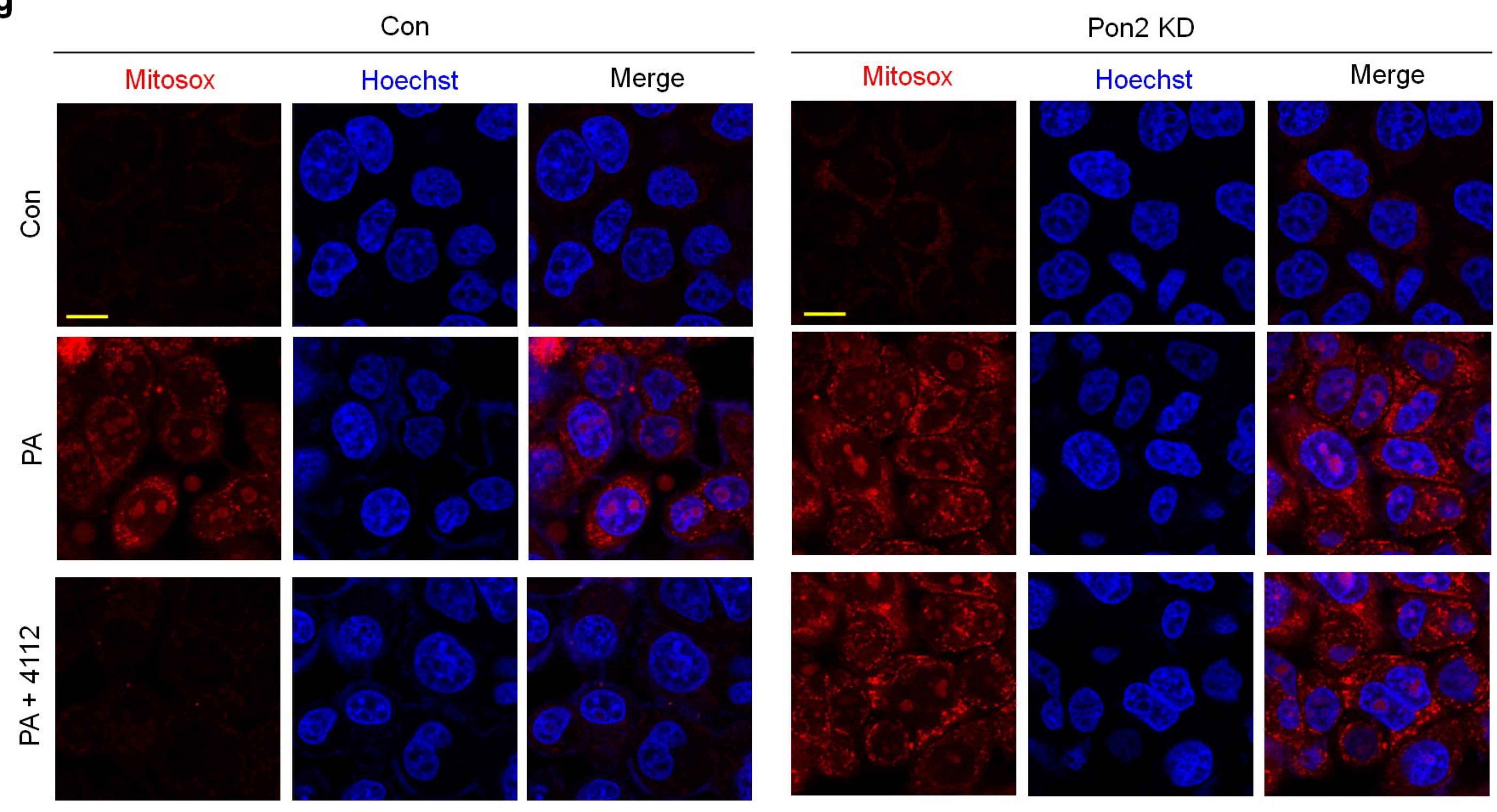

- Con

- ${ }_{\mathrm{PA}}^{\mathrm{PA}}+4112$

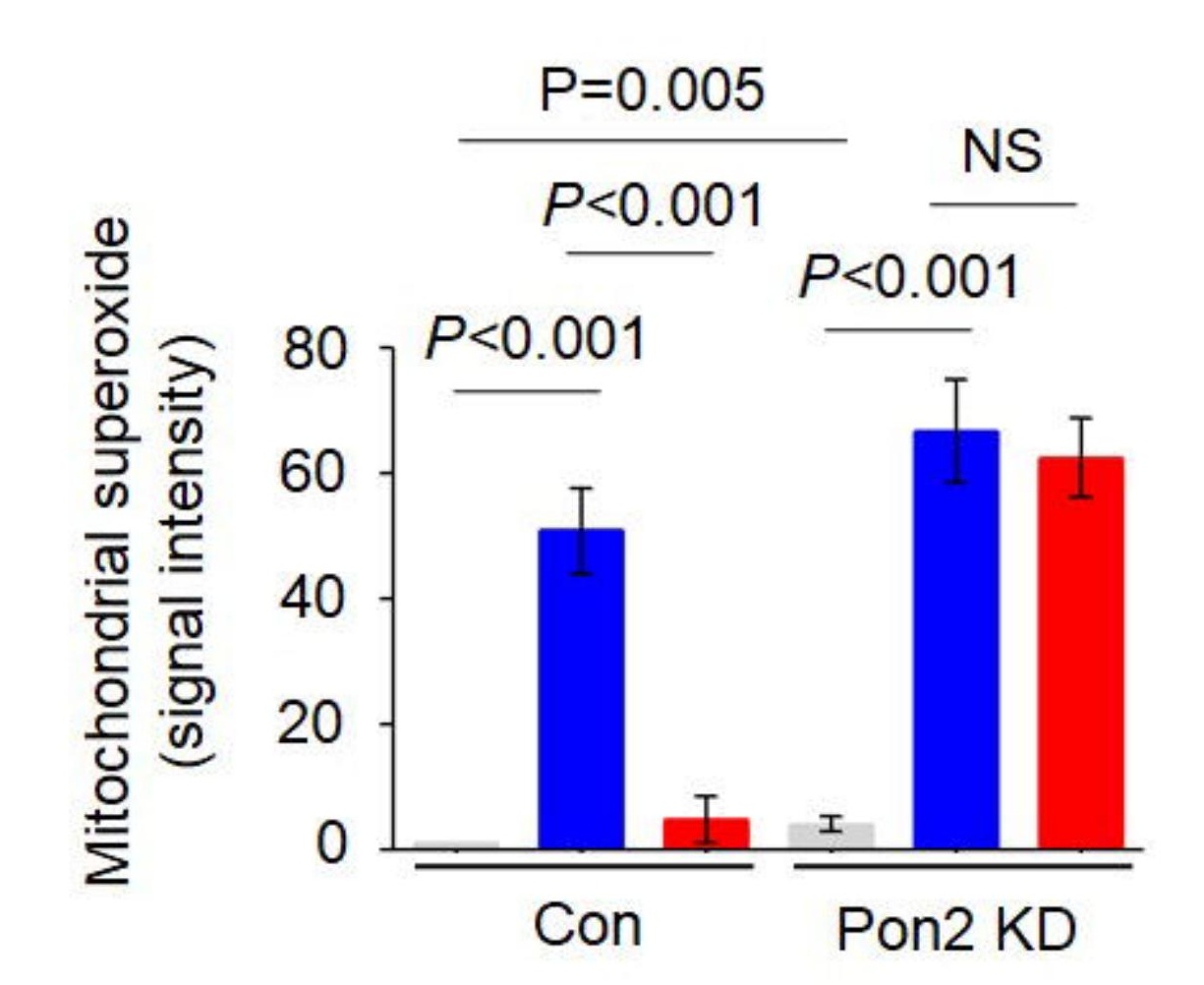

h

Con
PA
$P A+4112$
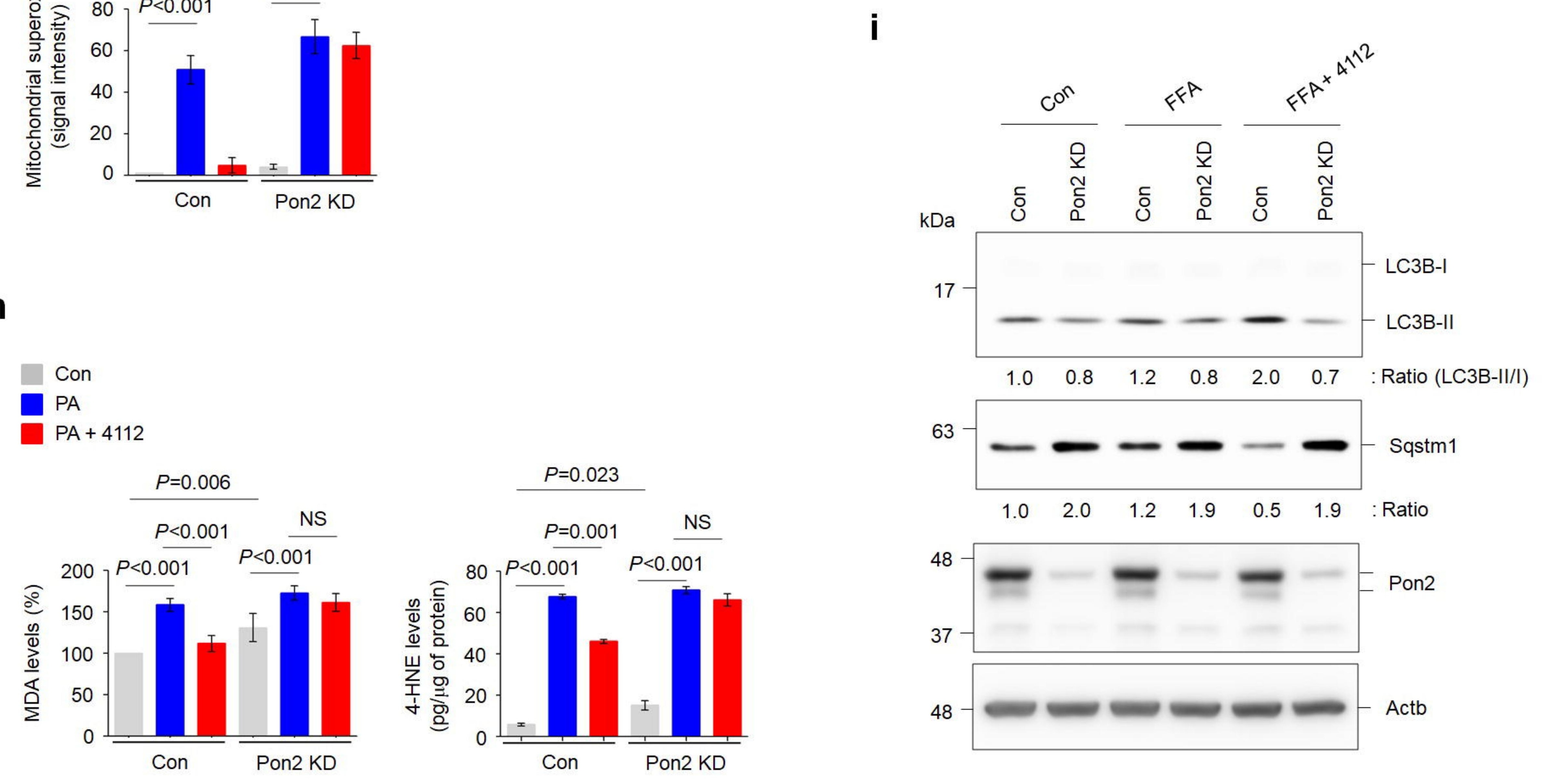

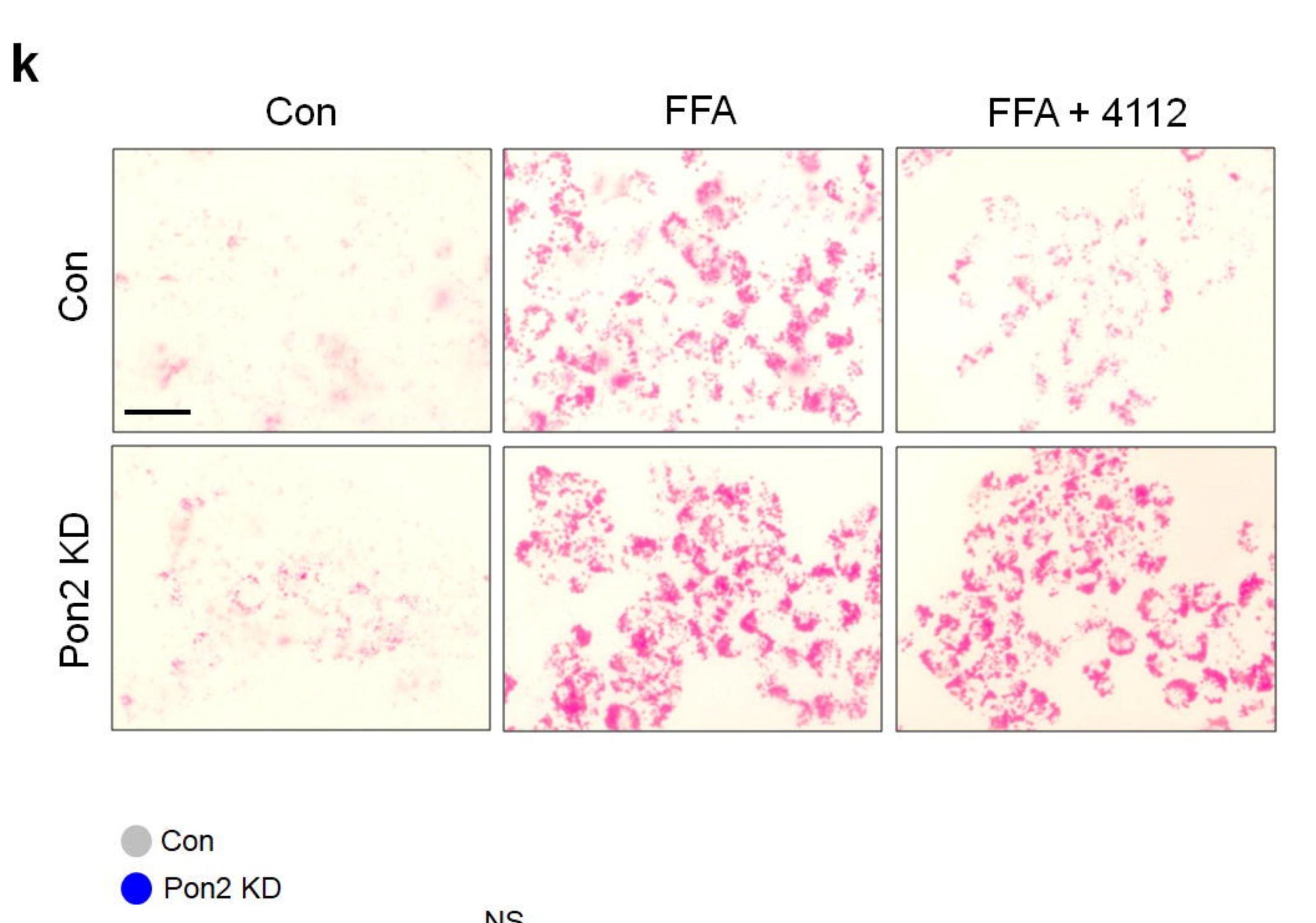

Con
- Pon2 KD

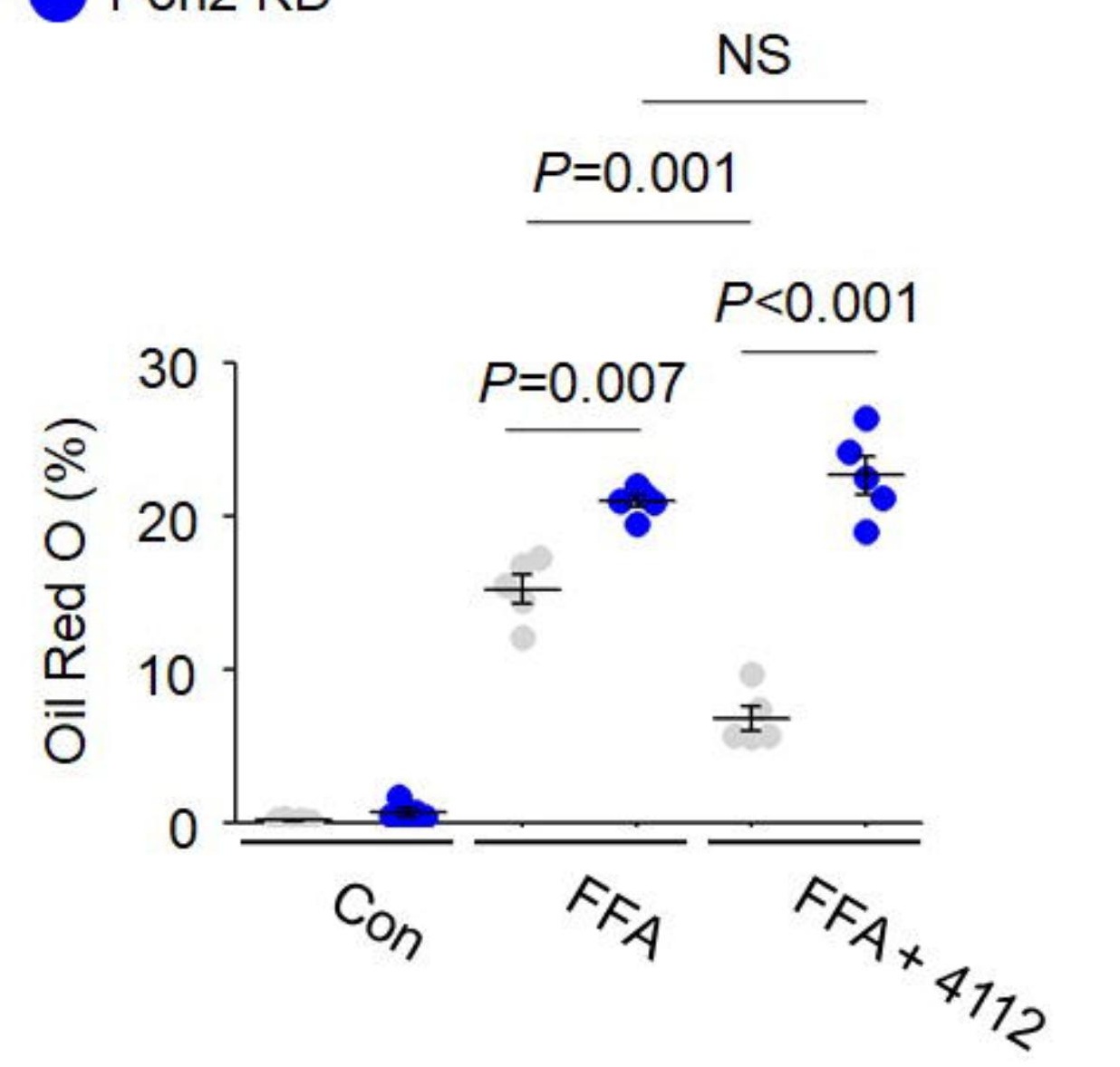

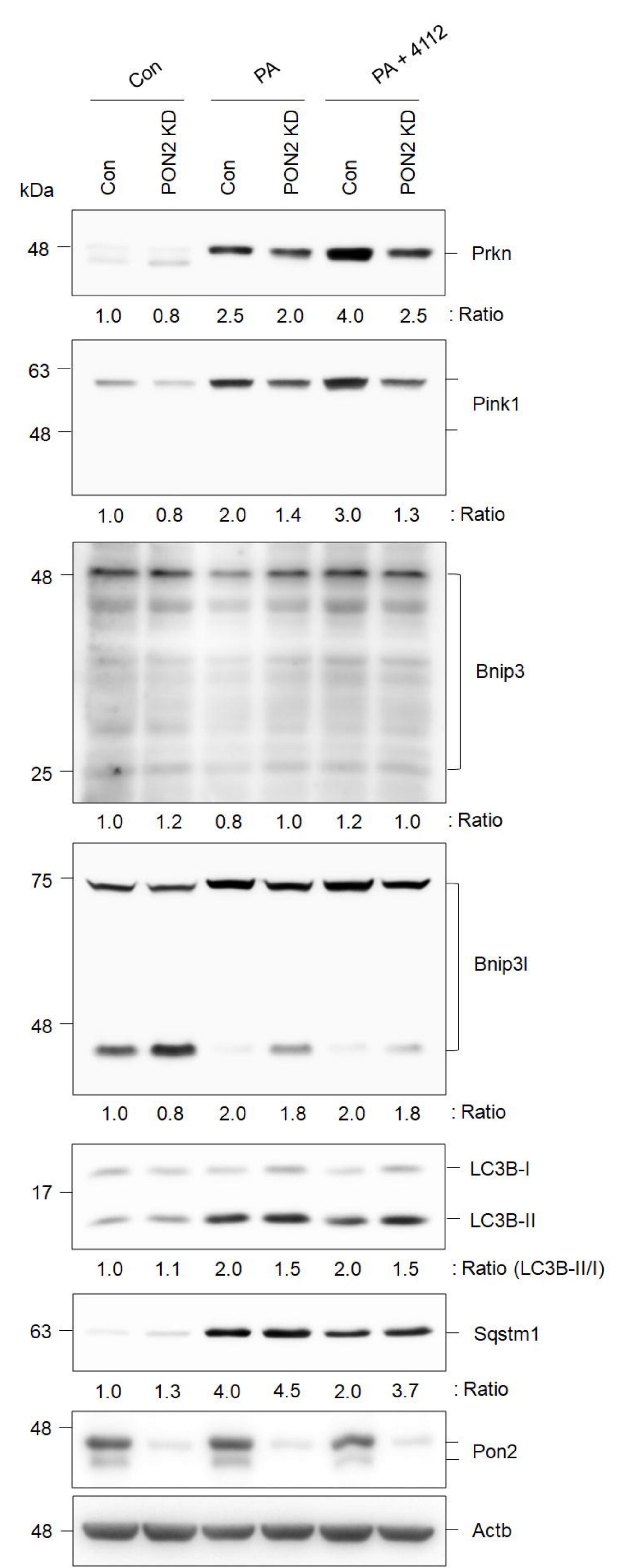

